

\section{Overlimiting current properties at ion exchange membranes}

\section{De eigenschappen van overlimiting current bij ionenuitwisselingsmembranen}

Joeri Carel de Valença 


\section{Promotiecommissie}

$\begin{array}{lll}\text { Voorzitter } & \text { Prof. dr. ir. J.W.M. Hilgenkamp } & \text { Universiteit Twente } \\ \text { Promotor } & \text { Prof. dr. ir. R.G.H. Lammertink } & \text { Universiteit Twente } \\ \text { Copromotor } & \text { Dr. ir. R.M. Wagterveld } & \text { Wetsus } \\ \text { Overige leden } & \text { Prof. dr. ir. M. Wessling } & \text { RWTH Aachen University } \\ & \text { Prof. H. Bruus } & \text { Technical University of Denmark } \\ & \text { Prof. dr. S.G. Lemay } & \text { Universiteit Twente } \\ & \text { Prof. dr. J.C.T. Eijkel } & \text { Universiteit Twente } \\ & \text { Dr. ir. W.M. de Vos } & \text { Universiteit Twente }\end{array}$

This work was performed in the Soft Matter, Fluidics and Interfaces (SFI) group at the $\mathrm{MESA}^{+}$institute for nanotechnology at the University Twente and in the cooperation framework of Wetsus, European Centre of Excellence for Sustainable Water Technology (www.wetsus.eu). Wetsus is co-funded by the Dutch Ministry of Economic Affairs and Ministry of Infrastructure and Environment, the Province of Fryslân, the Northern Netherlands Provinces. This work is part of the research program of RUG/Campus Fryslân and financed by the Province of Fryslân.

\section{Overlimiting current properties at ion exchange membranes}

ISBN: 978-90-365-4314-9

DOI: $10.3990 / 1.9789036543149$

URL: https://doi.org/10.3990/1.9789036543149

Typeset: LATEX

Printed by: Gildeprint

Cover photo: "tracer particles near structured membrane $(L=400 \mu \mathrm{m})$ "

Copyright (C) 2017 by Joeri de Valença 


\section{OVERLIMITING CURRENT PROPERTIES AT ION EXCHANGE MEMBRANES}

\section{PROEFSCHRIFT}

ter verkrijging van

de graad van doctor aan de Universiteit Twente, op gezag van de rector magnificus, prof. dr. T.T.M. Palstra,

volgens besluit van het College voor Promoties

in het openbaar te verdedigen

op vrijdag 10 Maart 2017 om 14:45 uur

door

Joeri Carel de Valença

geboren op 16 Januari 1985

te Haarlem, Nederland 
Dit proefschrift is goedgekeurd door:

Prof. dr. ir. R.G.H. Lammertink

and

Dr. ir. R.M. Wagterveld 
"Say it straight, simple and with a smile." "Know that one day your pain will become your cure."

"Life expands according to one owns courage."

- Yogi Tea 



\section{Contents}

1 Introduction $\quad \mathbf{1 1}$

1.1 Ion exchange membranes . . . . . . . . . . . . . . . . . 13

1.2 Electrodialysis . . . . . . . . . . . . . . . . . . . 14

1.3 Diffusion, Migration and Advection: Nernst-Planck, Poisson, Navier-

Stokes equation . . . . . . . . . . . . . . . . . . 18

1.4 Ion concentration polarization . . . . . . . . . . . . . . . . 21

1.5 Limiting current density . . . . . . . . . . . . . . . . . . 24

1.6 Time-dependent ion concentration polarization . . . . . . . . . 25

1.7 Overlimiting current . . . . . . . . . . . . . . . 27

1.7.1 Electroconvection . . . . . . . . . . . . . . 29

1.7.2 Gravitational convection . . . . . . . . . . . 30

1.8 Scope of thesis . . . . . . . . . . . . . . . . . . . 32

2 Experimental methods $\quad \mathbf{4 3}$

2.1 Electrochemical measurements . . . . . . . . . . . . . . . . 43

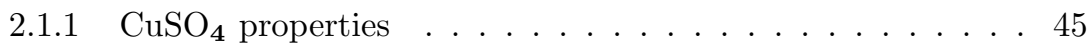

2.1.2 Membrane test cell . . . . . . . . . . . . . . . . 45

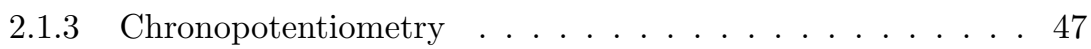

2.1.4 Chronoamperometry . . . . . . . . . . . . 50

2.1.5 Linear voltage sweep . . . . . . . . . . . . . . 51

2.2 Flow measurements . . . . . . . . . . . . . . . . . 51

2.2.1 Particle Image Velocimetry (PIV) . . . . . . . . . . . 53

2.2.2 Flow velocity errors induced by tracer particles . . . . . . 55

2.2.3 Flow velocity errors induced by vector field analysis . . . . 56

2.3 Concentration measurements . . . . . . . . . . . . . . . 58

2.3.1 Fluorescence Lifetime Imaging Microscopy (FLIM) . . . . . 61

2.4 Experimental techniques used in each chapter . . . . . . . . . . . . 62

3 Dynamics of Micro-vortices Induced by Ion Concentration Polarization $\mathbf{7 1}$

3.1 Introduction . . . . . . . . . . . . . . . . 72

3.2 Material and methods . . . . . . . . . . . . . . . . . 72

3.2.1 Particle Image Velocimetry Analysis . . . . . . . . . . . . . 74 
3.3 Results and discussion . . . . . . . . . . . . . . . 76

3.3.1 Chronopotentiometric response of overlimiting current . . . 77

3.3.2 Dynamics of micro-vortices during overlimiting current . . . 79

3.3.3 Viscous dissipation within micro-vortices . . . . . . . 82

3.4 Conclusion . . . . . . . . . . . . . . . . . . . . 83

3.A Extra experimental details . . . . . . . . . . . . . . . . 85

3.B Discussion on the error in the vector field analysis . . . . . . . . 86

3.C Supporting movies ... . . . . . . . . . . . . 87

4 Effect of the gravitational orientation on the overlimiting current 93

4.1 Introduction . . . . . . . . . . . . . . . . . 94

4.2 Material and methods . . . . . . . . . . . . . . . . 95

4.2.1 Electrochemical measurements (Chronopotentiometry) . . . 95

4.2.2 Flow measurements (PIV) . . . . . . . . . . . 96

4.2.3 Concentration measurements (FLIM) . . . . . . . . . . 97

4.2.4 Numerical model of concentration dynamics . . . . . . . . . 98

4.3 Results and discussion . . . . . . . . . . . . . . . . . . . . . . . . . . . . . . . . . . . . . . . .

4.3.1 Steady state. . . . . . . . . . . . . . . 99

4.3.2 Transition time . . . . . . . . . . . . . 101

4.3.3 Concentration profile development . . . . . . . . . . 104

4.4 Conclusion . . . . . . . . . . . . . . . . 106

4.A Supplemental movies . . . . . . . . . . . . . . . . 107

5 Confined electroconvective vortices at structured membranes $\quad \mathbf{1 1 3}$

5.1 Material and methods . . . . . . . . . . . . . . 115

5.1.1 Membrane preparation and pretreatment . . . . . . 116

5.1 .2 Experimental setup . . . . . . . . . . . . . 116

5.2 Results and discussion . . . . . . . . . . . . . . . . 118

5.2.1 The onset of electroconvection . . . . . . . . . . . 118

5.2.2 The growth of electroconvection . . . . . . . . . . . . 120

5.2.3 The saturation of electroconvection . . . . . . . . . . 123

5.2 .4 Ion flux calculations . . . . . . . . . . . . . . . . . 125

5.2.5 Numerical concentration and flow calculation . . . . . . 127

5.3 Conclusion . . . . . . . . . . . . . . . . . . 130

6 Summary and outlook 137

6.1 Summary . . . . . . . . . . . . . . . . . . 137

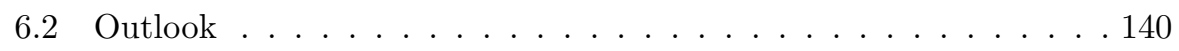

6.2.1 Membrane shape optimization . . . . . . . . . 141

6.2.2 Comparison between numerical and experimental work . . . 143 
6.2.3 Reduced resistance due to electroconvection . . . . . . . . 147

6.2.4 Final remarks on applications with overlimiting current . . 149

Algemene Nederlandse samenvatting 



\section{CHAPTER 1}

\section{Introduction}

To add salt to water is not complex, however to remove salt from water exposes a challenge. This thesis addresses this challenge by focusing on salt transport in water under an applied electric field across an ion exchange membrane.

Salty taste of food is attributed to the amount of salt added or already present in the food itself; in other words it is attributed to the salt concentration. To dissolve common kitchen salt into water is a favorable process so no applied energy is required unless the amount of salt is higher than the solubility capacity of the system. Water can actually contain up to $359 \mathrm{~g} / \mathrm{L}$ of common kitchen salt, $\mathrm{NaCl}$ at room temperature conditions $\left(\mathrm{T}=25{ }^{\circ} \mathrm{C}, \mathrm{P}=1 \mathrm{~atm}\right)$ [1]. Sodium chloride $(\mathrm{NaCl})$ in water dissociates into the positively charged cation, $\mathrm{Na}^{+}$, and negatively charged anion, $\mathrm{Cl}^{-}$. The electric charge of the ions can be used to manipulate the salt ions inside the water, because cations and anions migrate in opposite directions along an electric field which is produced by an external energy source [2].

An ion exchange membrane (IEM) is made from a chemically tuned polymeric material with fixed charged groups that rejects ions with the same charge (co-ions) and allows opposite charged ions (counter-ions) to pass through. A perpendicular electric field to a flat IEM will force the counter-ions through, while it blocks the co-ions, that are forced in the opposite direction. One side will get enriched of ions, while the other side gets depleted, see figure 1.1. This separation of salt with an external electric field is used in electrodialysis to desalinate different type of streams [2].

In electrodialysis, a feed solution flows along flat cation exchange membranes $(\mathrm{CEM})$ and anion exchange membranes (AEM) that are stacked in an alternate order between two opposite charged electrodes. The electric field causes ion migration and the compartments between the membranes get alternately enriched and depleted of ions. Initially the system follows Ohm's law with a linear relation between applied voltage (electric energy) and resulting current (desalination rate) [2]. At a certain electric field, the depleted side of the membrane approaches an ion concentration close to zero which increases the system resistance signifi- 


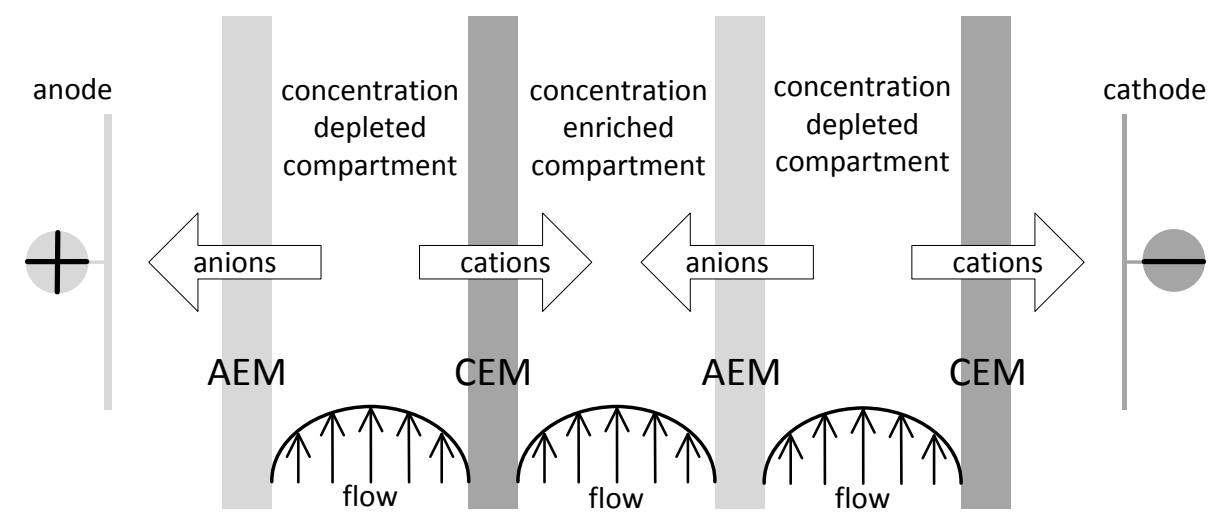

Figure 1.1: Schematic picture of an electrodialysis setup. A feed solution flows between membrane compartments made by alternating CEM and AEM. An applied electric field causes anions to migrate to the anode and cations to the cathode. Due to the selectivity of the membranes a concentrated and depleted stream are achieved.

cantly. This is called the limiting current regime. The theory on limiting current is well established and provides an upper limit on current possible through the compartment. However, if the electric field is increased above a threshold voltage, the current increases again reaching the overlimiting current (OLC) regime. Additional ion transport mechanisms (e.g. electroconvection or water dissociation) start to play an important role which make these mechanisms of theoretical and practical interest [3]. In the overlimiting regime, the coupling between the electric field and the depleted membrane interface presents a multitude of theoretical and experimental challenges [4-6]. The application of overlimiting current is attractive due to its ion transport enhancement, therefore faster desalination capacity per membrane surface area, which is the most expensive part of the electrodialysis system [2].

This thesis presents the investigation of the overlimiting current by combining electrical resistance, fluid flow and salt concentration measurements near the membrane surface. A model is presented to describe the change in concentration at given applied current density. This model allows to estimate when the system becomes unstable and overlimiting current can start. To achieve the goal of this thesis, three main aspects need to be addressed. The first aspect is to figure out how to measure the OLC properties using optical methods. The second aspect is to use this to create a better understanding of the process. The last aspect is to design membranes that have enhanced overlimiting current properties. Membranes with a geometrical structure (undulated membranes) showed a lower resistance than flat membranes in the overlimiting current condition, probably 
due to enhanced electroconvective mixing at the periodic structured interface $[4,7]$.

In the next sections a deeper scientific introduction into this topic is given. The relevant physical properties of the system are discussed and the assumptions behind the mathematical formulations are given. At the end of this chapter an outline of the thesis will be given.

\subsection{Ion exchange membranes}

A membrane is generally defined as "a selective material barrier that separates and/or contacts two adjacent phases and allows or promotes the exchange of matter between the phases" [8]. Most membranes exclude on size, which is determined by the pore size of the material. When ions are dissociated in water, van der Waals forces (attraction between ions and water molecules) lead to solvation and therefore to hydration shells [1]. The effective size of an ion, the Stokes radius, is in the order of $0.1 \mathrm{~nm}$, indicated as $\mathcal{O}(0.1 \mathrm{~nm})$. This is the same order of magnitude as a water molecule [1]. Separation of ions and water is still possible using dense membranes in which the selectivity depends on diffusion and chemical interaction between the species and the membrane matrix [9]. Reverse osmosis membranes are selective for water, while ion exchange membranes are selective for positive or negative ions [8].

Positive ions (cations) and negative ions (anions) are attracted while equal charges repel each other due to electrostatic forces. This ensures charge separation only occurs on a small scale, called the Debye length, $\lambda_{D}$. In salt water $\lambda_{D}=\mathcal{O}(\mathrm{nm})$. At length scales above $\lambda_{D}$ the anion and cation concentration can be assumed equal, also called the local electroneutrality assumption [10]:

$$
\sum_{i} z_{i} c_{i}=0
$$

In a symmetric binary solution there are only two ion species, $i=+,-$, with valence number, $z_{+}=-z_{-}$, the concentration of cations and anions is therefore everywhere the same, $c_{+}=c_{-}$.

An ion exchange membrane can be made of a polymeric material with a high concentration of immobile fixed charges. In figure 1.2 a homogeneous ion exchange membrane is shown in which the fixed charges are attached to a polymer backbone. To ensure electroneutrality inside the membrane opposite charged mobile counter-ions are present in a higher concentration than the similar charged 
mobile co-ions [8]:

$$
\sum_{i} z_{i} c_{i}+\chi=0
$$

If the fixed charge is positive, $\chi>0$, cations are rejected making the membrane an anion exchange membrane (AEM). Typically the membrane backbone is made of a polymer and the fixed groups are nitrogen, phosphor or sulphur based: $-\mathrm{NH}_{3}{ }^{+},-\mathrm{NRH}_{2}{ }^{+},-\mathrm{NR}_{2} \mathrm{H}^{+},-\mathrm{NR}_{3}{ }^{+},-\mathrm{PR}_{3}{ }^{+},-\mathrm{SR}_{2}{ }^{+}$. At a cation exchange membrane $(\mathrm{CEM}), \chi<0$, the fixed groups can be $-\mathrm{SO}_{3}{ }^{-},-\mathrm{COO}^{-},-\mathrm{PO}_{3}{ }^{2-}$, $-\mathrm{PO}_{3} \mathrm{H}^{-},-\mathrm{C}_{6} \mathrm{H}_{4} \mathrm{O}^{-}$, etc. See the review of $\mathrm{Xu}$ for more information on the chemistry of ion exchange membranes [11, 12].

The charge selectivity is used in a wide variation of systems to separate or exchange ions from liquids, gases, solids or a combination. The driving force of the separation process are most often electrical fields or concentration gradients. Electric driven processes include electrodialysis (for example food products demineralization or table salt production [2]), capacitive membrane desalination (for example for brackish water desalination [13]) and bipolar electrodialysis (for example for the production of acids and bases [14]). Concentration gradient driven processes are Donnan dialysis (for example for the removal of unwanted chemical [2]) or reverse electrodialysis (for the generation of electrical energy $[15,16])$. Ion exchange membranes are also found in electrochemical fuels cells $[17,18]$ and microbiological fuel cells [19].

\subsection{Electrodialysis}

The most common application of ion exchange membranes (IEM) is in electrodialysis (ED) for desalination of different types of streams [2, 20-22]. In a typical ED setup flat sheet anion exchange membranes (AEM) and cation exchange membranes (CEM) are stacked in an alternate order, see figure 1.3. An electric field is applied perpendicular to the membranes which drives anions to the anode and cations to the cathode. The compartments next to the electrode have a different electrolyte solution that host the electrochemical conversion of ionic current to electric current [23]. The other compartments get alternately depleted or enriched by ions. By pumping the feed solution through and collecting the outflow of alternating compartments, depleted and enriched streams are isolated, see figure 1.1 .

The product output can be defined by the desalination ratio, feed recovery, water recovery, current efficiency, energy consumption and operational costs. The main operational parameters are the feed composition, the flow rate, the cur- 


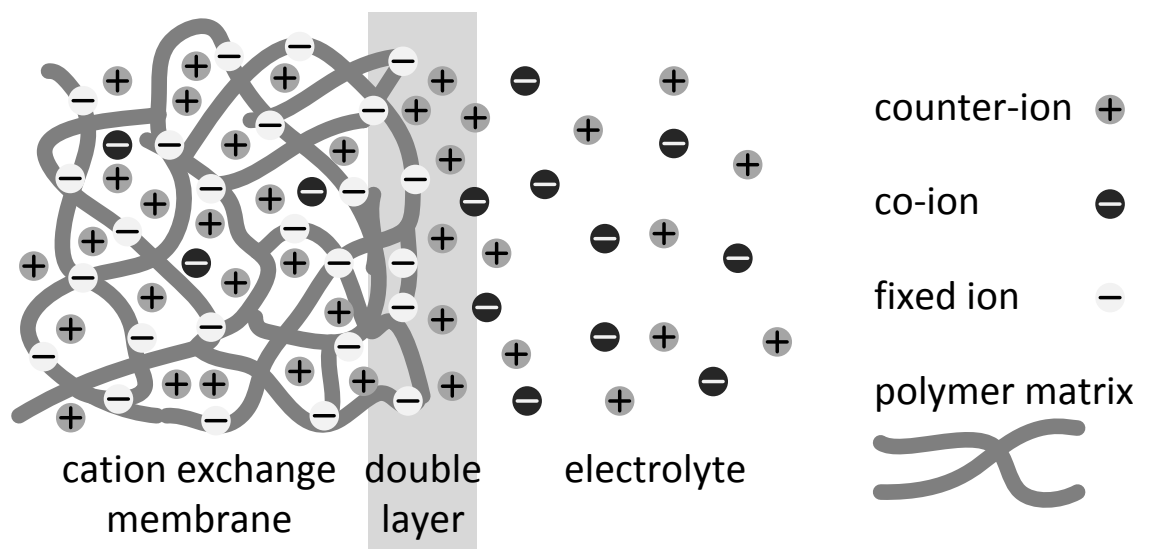

Figure 1.2: Schematic picture of a cation exchange membrane (CEM) with a charged polymer matrix and mobile counter-ions next to an electroneutral electrolyte. At the interface between the membrane and the electrolyte an electric double layer occurs. The ion concentration in the membrane, $\mathcal{O}(5 \mathrm{M})$, is much higher than in the electrolyte, $\mathcal{O}(0.001-1 \mathrm{M})$.

rent density, the membrane type and compartment geometry. The cell geometry, flow rate and feed composition determine the hydraulic resistance of the system, therefore the energy losses due to pumping. The amount of desalination is proportional to the applied current and the membrane selectivity. The transport of co-ions and water through the membrane reduces the system separation efficiency and is not desired $[2,24]$. The energy efficiency depends most on the resistances in the system. These can be divided into resistance of the membrane, the electrolyte and of the concentration potentials. The concentration potentials arise at the membrane interface (Donnan potential) and in a concentration gradient if the anion and cation have different diffusion constants (diffusion potential). For more information on these potentials the reader is referred to other work $[2,24]$. The energy loss due to the concentration potentials is in ED much lower than the energy loss due to the electrolyte resistance [2]. In electrodialysis membranes with a low electrical resistance are preferred, but the resistance of the membrane is generally lower than that of the depleted electrolyte [15, 25].

An essential property of electric fields is that they can be expressed as a gradient of a scalar potential [26]:

$$
\mathbf{E}=-\nabla V
$$

with $\mathbf{E}=\left(E_{x}, E_{y}, E_{z}\right)$ as the electric field vector, $\nabla=\left(\partial_{x}, \partial_{y}, \partial_{z}\right)$ as the vector 


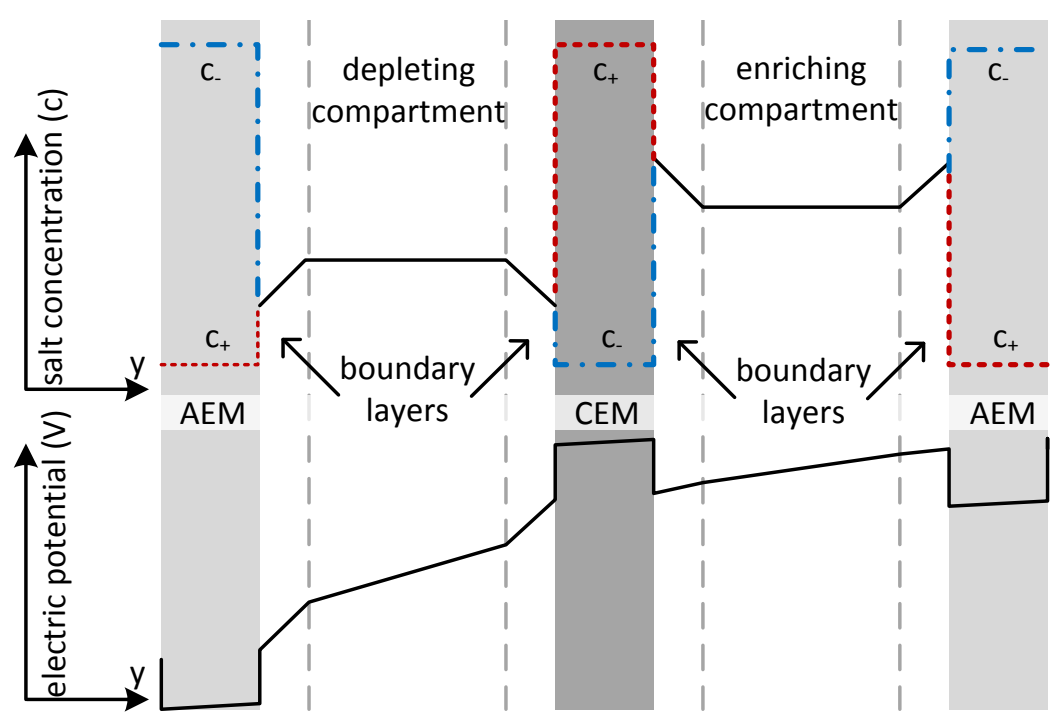

Figure 1.3: Top figure shows a schematic concentration profile in ED cell pair (not to scale). In the compartments the anion and cation concentration is the same due to electroneutrality, while in the membrane the concentration of mobile cations (red dotted line) and anions (blue dotted line) differs due to the presence of the fixed charges. An applied electric potential drop causes concentration polarization in the boundary layers, which depletes one compartment and enriches the other. The bottom figure shows schematically the potential distribution (not to scale). The potential drops most in the depleting compartment. In the boundary layers the potential drop is higher due to the diffusion potential. At the membrane interface a Donnan potential arises. If the concentration at both sides of the membrane differs a the sum of two Donnan potentials also known as the Nernst potential.

notation of the gradients in three dimensions of $V$, the electric potential. The relation between the electric driving force, the resulting current and the resistance is known as Ohm's law:

$$
\Delta V=R I
$$

with $\Delta V$ as the electrical potential drop over the system, $R$ as the resistance and $I$ as the current. In figure 1.3 a schematic of the voltage drop over a ED cell pair is shown. The resistance of an electrolyte is dependent on electrolyte resistivity, the distance between the electrodes and the cross sectional area of the 
material:

$$
R=\rho_{r} \frac{l}{A}
$$

with $l$ is the length, $A$ area and $\rho_{r}$ the resistivity. The resistivity depends on ion concentration. When more ions are present the electrolyte conducts better. Experimentally it is found that the conductivity depends almost linear on the ion concentration. Only at very high and low concentrations it deviates [1]:

$$
\sigma=\frac{1}{\rho_{r}} \sim c
$$

where $\sigma$ is the conductivity and $c$ the ionic concentration in the electrolyte. Figure 1.4 shows a typical current voltage response in an electrodialysis system. At low voltages the behaviour follows Ohmic law, having a constant $R$, but at higher applied cell voltages a current plateau (limiting current, $I_{\lim }$ ) is visible. This increase of resistance comes from a small depletion layer, thus low conductance (eq. 1.6). Although thin, this layer dominates the overall resistance.

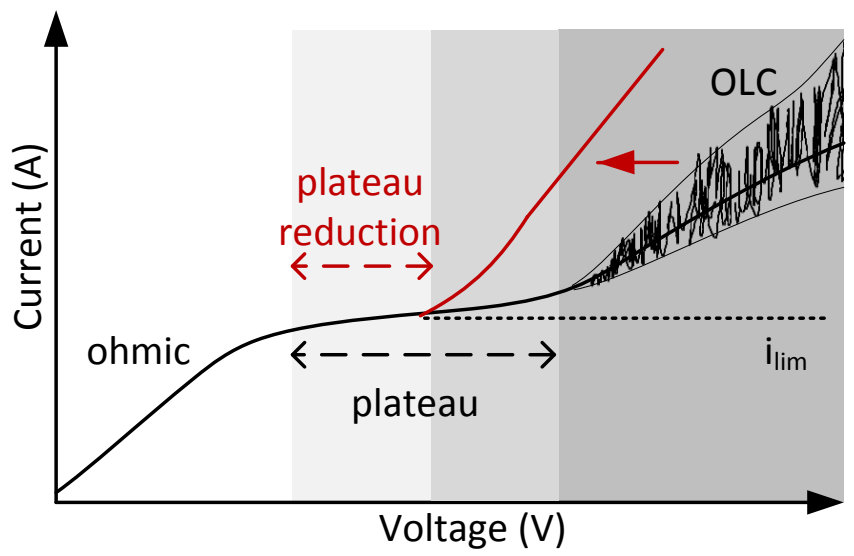

Figure 1.4: A picture of the typical voltage current response in an ED compartment. At low voltage the system shows an ohmic resistance. The interface depletion at the limiting current shows as a plateau regime. Above threshold voltage overlimiting current (OLC) mechanisms start increasing the total current. The current response typically fluctuates in the OLC regime. The fluctuations increase with higher voltage and the errorbar of the average is large. The red line indicates a desired reduction in OLC resistance.

In practice an ED system is operated just below the limiting current density [14]. In desalination technologies ED distinguishes itself when small scale application are needed [14]. Another advantage is that it selectively removes the 
salt out of the solution, which gives it an advantage over other techniques in which the water is removed, e.g. reverse osmosis or distillation [8, 27]. When low amount of salt needs to be removed from the water, e.g. brackish water desalination, ED is a good choice [14]. Also when salt needs to be removed from a multicomponent mixture (e.g. with non-charged proteins like cheesy whey of soy sauce) ED is beneficial [21, 28]. A desirable application for ED is selective removal of ions $[3,29,30]$. Fouling occurs in all membrane systems, but in ED the current can be reversed to remove fouling [31, 32].

A disadvantage of ED is that chemical fouling inside the membrane can occur which increases the membrane resistance $[2,32]$. At large scale seawater desalination RO is more efficient [27], although seawater pre-concentration with ED is proposed to improve the process efficiency [33]. At high concentration the resistance electrolyte resistance is very low, thus the resistive losses are low. Another disadvantage are the expensive ion exchange membrane. Progress is made by decreasing the production costs [34].

Increasing the current density is also a way to reduce the necessary membrane area while achieving the same amount of desalinated product. Common practice is to operate close to the limiting current density [14]. As illustrated in figure 1.4 the limiting current is not really the maximum current. Increasing the voltage even further can result an increase in current, named overlimiting current (OLC) [3]. This region is avoided for two reasons. Water dissociation might occur at the membrane and the energy efficiency of the current transfer decreases. However, research indicate that the water dissociation hardly occurs at cation exchange membranes [35] and that the overlimiting current resistance can be decreased by geometrically shaped membranes, as indicated in figure 1.4. The design of such membranes is a part of this work. To understand what is occurring in the OLC region, in the next section the ion transport dynamics are discussed, specifically at the depleted side of the membrane.

\subsection{Diffusion, Migration and Advection: Nernst-Planck, Poisson, Navier-Stokes equation}

The three main mechanisms in ion transport are: (a) advection due to a mechanical force on the liquid, (b) diffusion due to a gradient in concentration, and (c) migration due to an external electric force on the charged ions. When the coupling between the fluxes is neglected they can be combined in the Nerst-Planck flux equation with units $J=\left[\mathrm{mol} / \mathrm{s} / \mathrm{m}^{2}\right][10]$ : 


$$
\begin{aligned}
\mathbf{J} & =\mathbf{J}_{a d v}+\mathbf{J}_{d i f}+\mathbf{J}_{e l} \\
\mathbf{J}_{i} & =c_{i} \mathbf{v}-D_{i} \nabla c_{i}-D_{i} \frac{z_{i} e}{k_{B} T} c_{i} \nabla V \\
i & =+,-
\end{aligned}
$$

In a simple binary systems the cation flux, $J_{+}$and anion flux, $J_{-}$are carried by two ionic species. Cases with mixtures of electrolytes are discussed in other references [36].

The advective flux is given by the concentration $c_{i}$ and fluid velocity of the water, v. The flow distribution depends on the system geometry, the friction at the walls and driving forces.

The diffusive flux is written down as Fick's first law, the diffusion constant $D_{i}$ times the concentration gradient $\nabla c_{i}$. An ideal, sufficiently diluted electrolyte solution is implied. The chemical potential gradient, the driving force of diffusion, is dependent on concentration. The diffusion coefficient is assumed to be independent on concentration and advection and migration flux.

The migration term relates the electrical potential gradient, $\nabla V$, to the ions with charge sign dependent on the valence number $z_{i}$. In binary electrolytes $z_{+}=-z_{-}$and the flux of cations is opposite to the flux of the anions. The remaining symbols are the elementary charge, $e$, Boltzmann constant, $k_{B}$, and the temperature, $T$, which describe the interaction of the ions with the electric field $(\mathbf{E}=-\nabla V)$.

The potential gradient can be obtained from Poisson's equation:

$$
-\varepsilon \nabla^{2} V=\rho_{\mathrm{e}}=e|z|\left(c_{+}-c_{-}\right)
$$

where $\varepsilon$ is the electrical permittivity of the electrolyte and $\rho_{\mathrm{e}}$ the charge density, which occurs if there is a difference in positive and negative charges. Typically the difference in charge between electrodes causes the electric field. But at the membrane interface an electric double layer exists with a potential drop between it, see 1.3 .

The Navier-Stokes equation describes the conservation of momentum within the fluid under body and surface forces [10, 37]:

$$
0=-\nabla p+\mu \nabla^{2} \mathbf{v}+\rho_{\mathrm{e}} \mathbf{E}+\rho_{\mathrm{m}} \mathbf{g}
$$

where the pressure is $p$, and (dynamic) viscosity is $\mu$. The two body forces shown here are the electrical body force and the gravitational body force (with mass density, $\rho_{\mathrm{m}}$, and gravitational acceleration, $\mathrm{g}$ ). At low flow velocities the 
momentum inertia term $\left(\rho_{\mathrm{m}}[\mathrm{d} \mathbf{v} / \mathrm{d} t+(\mathbf{v} \cdot \nabla) \mathbf{v}]\right)$ is small compared to viscous term and can be neglected [37, 38].

In case of aqueous solutions the liquid is incompressible:

$$
\nabla \cdot \mathbf{v}=0
$$

The Nernst-Planck, Poisson and Navier-Stokes form a set of strongly coupled equations via the ion concentration, fluid velocity and electric potential. To solve the set of equation often only numerical solutions exist [37, 39]. However, if the flow is laminar analytical solutions of the flow profile exist. Pressure driven flow between two no-slip boundaries (i.e. boundaries where the friction with the solid reduces the flow velocity to zero) results in a parabolic flow profile known as the Hagen-Poisseuille flow [10, 38]. Also for simple cases where the flow is driven by the electric field via a charged interface layer analytical solutions exist. This phenomena is known as electro-osmosis, which will have the fastest flow speed at the interface [38]. More detail and more complex coupling between the charged layer and the electrical field be explained in more detail in section 1.7.1 in this thesis.

In experimental systems it is common to measure the current density through a system. This is the sum of the flux densities taking their charges into account:

$$
\mathbf{i}=F\left(z_{+} \mathbf{J}_{+}+z_{-} \mathbf{J}_{-}\right)
$$

The Faraday constant $\left(F=e N_{a}=9.65 \cdot 10^{4} \mathrm{C} / \mathrm{mol}\right)$ represents the amount of charge a mol of particles $\left(N_{a}=6.0 \cdot 10^{23}\right)$ with charge $\left(e=1.6 \cdot 10^{-19}\right)$ has. If the local electric field gradient $(\nabla V)$ is unknown, but the total current density (i) can be measured it is useful to rewrite the Nernst-Planck equation, eq. 1.8, through inserting it in the current density, eq. 1.13, using the electroneutrality assumption, eq. 1.1. This gives an expression for the electric field gradient which can be used to rewrite the Nerst-Planck equation to:

$$
\mathbf{J}_{i}=c_{i} \mathbf{v}-D \nabla c_{i}+\frac{\mathbf{i} t_{i}}{z_{i} F}
$$

where the diffusive flux now depends on the total salt diffusion constant, $D=$ $\left(z_{+}+\left|z_{-}\right|\right) D_{+} D_{-} /\left(z_{+} D_{+}+\left|z_{-}\right| D_{-}\right)$. And the transport number reflects the part of the current carried by each species $t_{i}=D_{i} /\left(D_{+}+D_{-}\right)$.

An ideal cation exchange membrane does not allow anions to pass, thus the $\overline{t_{+}}=1$ and $\overline{t_{-}}=0$. Commercial ion exchange membranes have a counter-ion transport number of $\bar{t} \approx 0.95$ [25]. Due to the high counter-ion concentration the gradient inside the membrane is small and diffusive transport can be neglected. 
Also advective transport through the dense membrane can be neglected due to high friction with the polymer [8].

\subsection{Ion concentration polarization}

Upon applying an electric field perpendicular to an ion exchange membrane counter-ions are forced though the membrane, while co-ions are forced in opposite direction. This is schematically shown for the interface at the depleted side of the membrane in figure 1.5a. Since the electrical ion fluxes inside the membrane and the liquid are not equal, the concentration at the interface will change in time, as expressed by the continuity equation:

$$
\nabla \cdot \mathbf{J}_{i}+\frac{\partial c_{i}}{\partial t}=0
$$
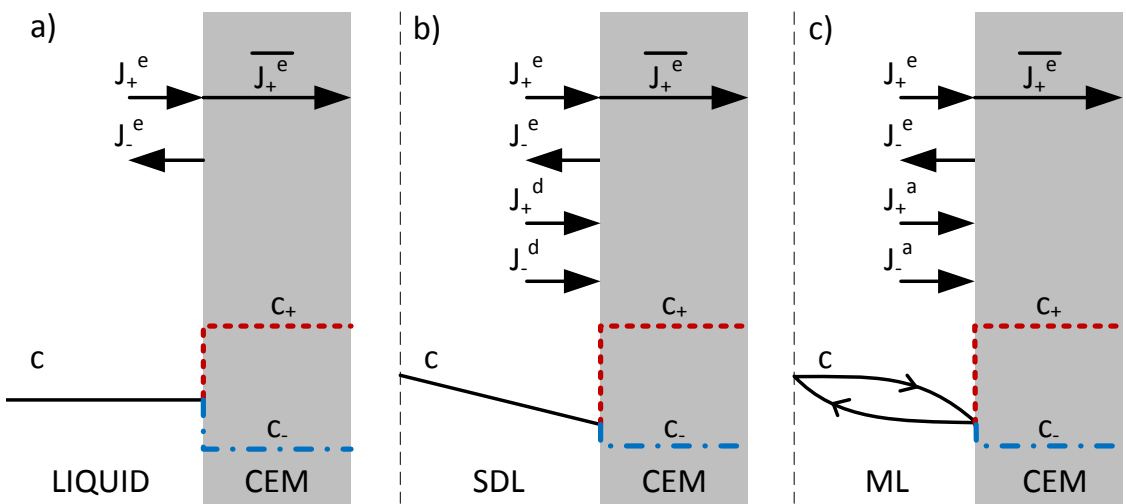

Figure 1.5: a) The selectivity of the membrane causes an imbalance in electromigration flux at the interface between membrane and liquid. b) A stagnant diffusion layer (SDL) can cause additional diffusive flux that balances the flux at the interface. c) A mixing layer $(\mathrm{ML})$ can cause additional advective flux that balances the flux at the interface. It is assumed that the inflowing liquid has a higher ion concentration than the outflowing liquid.

The continuity equation follows from the conservation of charge together with assumption that the salt stays completely dissolved. In other words that there are no chemical reactions, precipitation and aggregation. This is valid at low ion concentration for which the properties are described by the infinite solution limit [1].

The emerging concentration gradients at the interfaces enables diffusive transport of co- and counter-ions allowing the gradient to expand the gradient into the 
liquid. This gradient, called ion concentration polarization (ICP), corresponds to a depletion of ions towards one side of the membrane and in an enrichment of ions towards the other side, see figure 1.6.

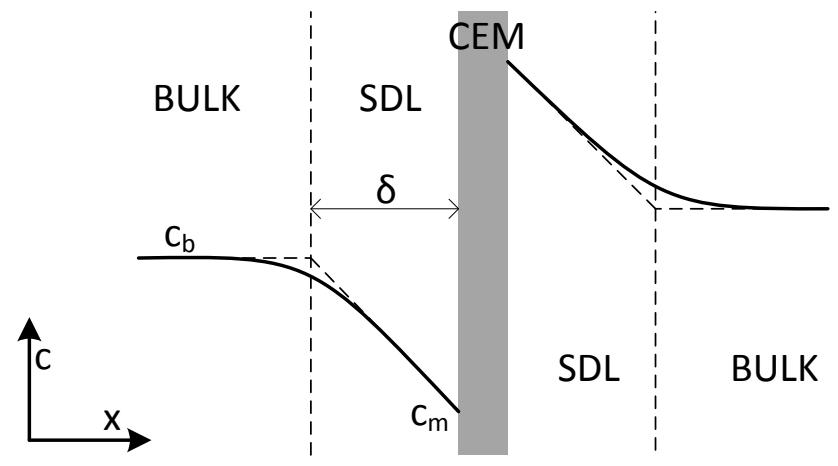

Figure 1.6: Schematic of concentration polarization along the direction of the current, $x$. The dashed lines represent stagnant diffusion layer (SDL) model.

Figure 1.5b shows schematically how diffusional flux of co- and counter-ions to the interface of the depleted side of the membrane can make the fluxes inside and outside the membrane equal. Figure $1.5 \mathrm{c}$ shows that also advection can fulfill this role to bring the system into steady state.

In electrodialysis, the concentration depletes continually along the flow (taken as the $y$ direction) and towards the membranes (taken as the $x$ direction), see figure 1.7. The concentration profile depends on the geometry and operation of the system. In ideal rectangular compartments no change in the $z$ direction is expected. The development of the concentration profile can be calculated directly by solving the Nernst-Planck equation (eq. 1.8 or eq. 1.14), together with the electroneutrality assumption (eq. 1.1) and continuity equation (eq. 1.15) using the proper boundary conditions [40].

The steady state concentration profile in an ideal rectangular compartment with a parabolic flow profile and constant current boundary condition, see figure 1.7b. To achieve a constant current, the voltage drop has to increase along the $y$ axis due to a decrease in concentration and thus an increase in resistance. Similarly a constant voltage boundary condition will result in a decreasing current along the $y$ axis. Numerical solutions of a rectangular compartment with a parabolic laminar flow profile and constant voltage drop boundary conditions are described by Tedesco et al. [41].

As can be observed in figure $1.7 \mathrm{~b}$ the concentration decreases from inlet to outlet achieving the lowest concentration at the membrane. The regions with 
a)

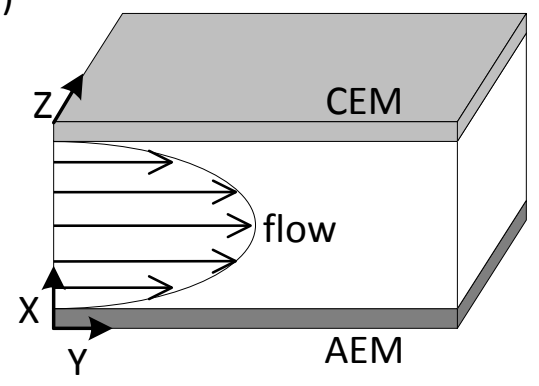

b)

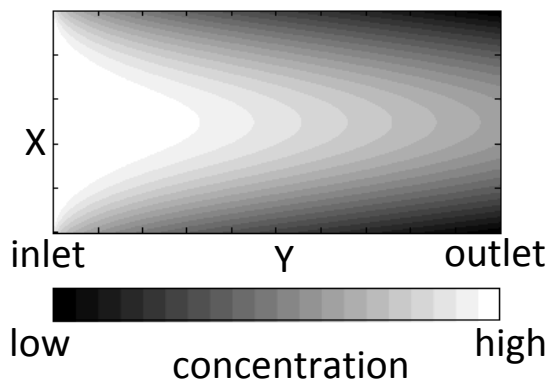

Figure 1.7: a) Model of rectangular flow compartment in electrodialysis with parabolic flow profile. b) Steady state solution of the concentration polarization in two dimensions in the depleted compartment.

low concentration dominate the resistance of the system. When the concentration reaches zero, the resistance of the system increases dramatically as the resistance is inversely proportional to the concentration (eq. 1.5). This is called the limiting current situation and occurs first at the end of the channel, see figure 1.4 for the typical current-voltage response of the system.

To reduce ICP often obstacles to induce flow in the $x$ direction are used. These obstacles are therefore called mixing promoters. Often they serve a second purpose of separating the membranes from each other and are therefore also referred to as spacers. The presence of these obstacles increases the complexity of the system. The occurrence of limiting current (visible from the increase in resistance in a current-voltage plot) can be used to determine the ion transport conditions inside the ED stack, since it gives an indication of the concentration at the membrane interface. Measurements inside and ED stack are difficult since the size of the channels makes it unapproachable for measurement probes, although some systems have been tried $[42,43]$.

The limiting current density can also be increased by increasing the flow rate. If the flow rate increases more ions are brought into the compartment, while a fixed amount, proportional to the current, is extracted through the membrane. The decrease in ion concentration along the $y$ axis is less, therefore the depletion at the end of the compartment occurs at higher current densities. This phenomena is also described by determining the concentration profile development, expressed via the Sherwood number [44, 45]. The dimensionless Sherwood number is the ratio between the actual mass transport rate $\left(k_{\mathrm{m}}[\mathrm{m} / \mathrm{s}]\right)$ and the diffusion rate 
$(D / L[\mathrm{~m} / \mathrm{s}]):$

$$
S h=\frac{k_{\mathrm{m}} L}{D}
$$

where $L$ is the typical length scale of diffusion and often taken as the compartment width, and $D$ is the diffusion constant. The actual mass transfer rate can be related to the current via $k_{\mathrm{m}} \sim i /\left(F \cdot c_{\mathrm{r}}\right)$, where $c_{\mathrm{r}}$ is some appropriate reference value for the ionic concentration $[44,46]$.

As an approximation of the real three dimensional process, a volume averaged Sherwood number is taken. This can be used to determine optimal operational conditions. Although the increase of flow rate and the placement of mixing promoters decrease ICP, thus the electrical resistance of the system (increase $i_{\text {lim }}$ ), they also increase the hydraulic resistance, thus the pumping energy. To find the optimal operational conditions often experiments are conducted with different flow, current and compartment geometry [22, 44, 46, 47].

\subsection{Limiting current density}

Independent of whether mixing promoters are used or not, the friction of the liquid with the membrane reduces the flow speed at the boundary [38]. The electric field drives the concentration change and the diffusion needs to balance the flux imbalance at the interface, see figure 1.5b. Fluxes through the boundary can be described with a one dimensional Nernst-Planck equation (eq. 1.14). The current and the concentration gradient are in the $x$ direction, thus $\mathbf{i}=\left(i_{x}, 0,0\right)$ and $\nabla c=\mathrm{d} c / \mathrm{d} x$.

$$
\begin{aligned}
\left.J_{i}^{l i q}(x)\right|_{x=0} & =-\left.D \frac{\mathrm{d} c(x)}{\mathrm{d} x}\right|_{x=0}+\left.\frac{i t_{i}}{z_{i} F}\right|_{x=0} \\
\left.J_{i}^{m e m}(x)\right|_{x=0} & =\left.\frac{i \overline{t_{i}}}{z_{i} F}\right|_{x=0}
\end{aligned}
$$

In steady state $J_{i}^{l i q}=J_{i}^{m e m}$, the following relation between current and interface concentration is found:

$$
i_{\text {boundary }}=\left.\frac{z_{i} F D}{\left(\overline{t_{i}}-t_{i}\right)} \frac{\partial c(x)}{\partial x}\right|_{x=0}
$$

Higher currents lead to a sharper gradient. The gradient at the boundary can be estimated by a linear extrapolation as seen in figure 1.6. The first order approximation of the gradient becomes $\partial c / \partial x=\left(c_{b}-c_{m}\right) / \delta$. The width of 
the diffusion layer $(\delta)$ depends on the shape of the concentration profile, which depends on the hydraulic resistance of the compartments, the flow speed, the transport numbers, current density and diffusion coefficient of the electrolyte. At the limiting current the concentration at the membrane vanished $\left(c_{m}=0\right)$, see figure 1.8a. This leads to the limiting current equation, also known as Peers equation [48]:

$$
i_{\text {lim }}=\frac{z_{i} F D}{\left(\overline{t_{i}}-t_{i}\right)} \frac{c_{b}-c_{m}}{\delta}=\frac{z_{i} F D}{\left(\overline{t_{i}}-t_{i}\right)} \frac{c_{b}}{\delta}
$$

It is important to realize that current, bulk concentration $\left(c_{b}\right)$ and stagnant diffusion layer (SDL) thickness change along the compartment ( $y$ direction) due to the continuous desalination. The limiting current condition occurs first at the end of the compartment where the bulk concentration is lowest. From current voltage measurements, like in figure 1.4, the limiting current can be determined.
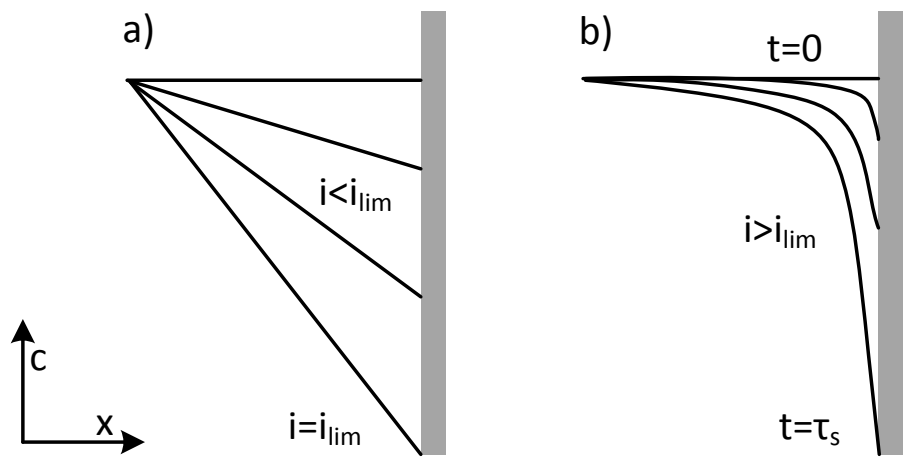

Figure 1.8: a) The steady state linear concentration gradient towards increases with applied current, with a maximum at the $i_{\text {lim }}$. b) If $i>i_{\text {lim }}$ the interfacial concentration will be depleted at the transition time $\tau_{s}$. The concentration profile will not be linear.

\subsection{Time-dependent ion concentration polarization}

This section describes the time-dependent depletion near a cation exchange membrane (CEM) within a stagnant, initially homogeneous electrolyte under constant current conditions. Once a current is forced through the system, the concentration near the interface depletes, forming a concentration gradient. Combining the continuity equation 1.15 and the Nernst-Planck equation 1.14 without flow 
and in one dimension leads to:

$$
\frac{\partial c_{i}}{\partial t}=-\frac{\partial}{\partial x}\left(-D \frac{\partial c_{i}}{\partial x}+\frac{i t_{i}}{z_{i} F}\right)
$$

The current density, $i$, does not depend on $x$ and the transport numbers only change at the interface. Inside the bulk the concentration of anions and cations is the same and the concentration profile is determined by the first term, which is known as Fick's second law of diffusion [10]:

$$
\frac{\partial c(x, t)}{\partial t}=D \frac{\partial^{2} c(x, t)}{\partial x^{2}}
$$

To solve this linear partial differential equation the boundary conditions need to be known. The initial condition is a homogeneous concentration $\left(c_{0}\right)$. The gradient at the boundary is governed by the set current, as derived in eq. 1.19 and the concentration at the end of the SDL $(x=\delta)$ is assumed to be the bulk concentration:

$$
\begin{aligned}
c(x, 0) & =c_{0} \\
\frac{\partial c(0, t)}{\partial x} & =-\frac{i}{z F D}\left(\overline{t_{+}}-t_{+}\right) \\
c(\delta, t) & =c_{0}
\end{aligned}
$$

It is possible to solve this set of equations numerically using a partial differential equation algorithm [49]. An analytical solution also exists and can be found using Laplace transformations $[50,51]$ :

$$
\begin{aligned}
c(x, t)=c_{0} & -\frac{i(\delta-x)\left(\overline{t_{+}}-t_{+}\right)}{z F D}+\frac{8 i\left(\overline{t_{+}}-t_{+}\right) \delta}{z F D \pi^{2}} \\
& \times \sum_{n=0}^{\infty} \frac{(-1)^{n}}{(2 n+1)^{2}} \sin \left[\frac{(2 n+1) \pi(\delta-x)}{2 \delta}\right] \exp \left[\frac{(2 n+1)^{2} \pi^{2} t}{4 \delta^{2}}\right]
\end{aligned}
$$

If the last term is zero the solution is time independent. This concentration profile can be any constant gradient. The steady state limiting current solution, as in eq. 1.19, can be obtained by setting $x=0$ and $c(x=0)=0$. Figure 1.8a shows schematically the steady state solutions and figure $1.8 \mathrm{~b}$ shows schematically the development in time of the concentration profile. When a current above the limiting current is applied the concentration at the membrane reaches zero before a steady state (constant gradient) is reached. 
The solution reduces in complexity if $\delta \rightarrow \infty$. The derivation can be found in [50]:

$$
c(x, t)=c_{0}-\frac{i\left(\overline{t_{+}}-t_{+}\right)}{z F D}\left[2 \sqrt{\frac{D t}{\pi}} \exp \left[\frac{-x^{2}}{4 D t}\right]-x \cdot \operatorname{erfc}\left[\frac{x}{2 \sqrt{D t}}\right]\right]
$$

Only when $i=0$ the equation has a time independent (steady state) solution, $c(x, t)=c_{0}$. Over an infinite diffusion layer no constant gradient solution is possible, since this would give an infinite concentration difference between both sides of the layer (membrane and bulk). From eq. 1.27 it is possible to extract an analytical expression for the time when the interface concentration is fully depleted, $c(x=0, t=\tau)=0$.

$$
c(0, \tau)=0=c_{0}-\frac{i\left(\overline{t_{+}}-t_{+}\right)}{z F D} 2 \sqrt{\frac{D \tau}{\pi}}
$$

which was first derived by Sand and therefore it is called the Sand's transition time [52]:

$$
\tau_{\mathrm{s}}=\frac{\pi D}{4}\left(\frac{c_{0} z F}{\overline{t_{+}}-t_{+}}\right)^{2} \frac{1}{i^{2}}
$$

Sand's equation is valid when no crossflow is present and the diffusion layer is smaller than the stagnant layer, $\delta$. This is valid at high currents $i>2 \cdot i_{\text {lim }}$ [53]. At lower currents the first order approximation of the diffusion equations can be used to get an analytical solution [54].

Sand's formula is used to correctly predict the depletion time in many constant current measurements $[55,56]$. Figure 1.9 shows a typical voltage response after applying a constant current through an initial homogeneous electrolyte and an ion selective surface. Once depletion occurs, the resistance increases drastically. Although the SDL theory predicts a layer fully depleted of ions with infinite resistance, experiments show a start of a new regime. Additional current transport mechanisms start, therefore rendering the model incomplete. The most important mechanisms causing the so-called overlimiting current are discussed in the next section.

\subsection{Overlimiting current}

Theories describing overlimiting current (OLC) can be divided in two different groups [3]. The first group relates the additional transport to physical advection of the salt ions in the boundary layer. Water with a high salt concentration flows 


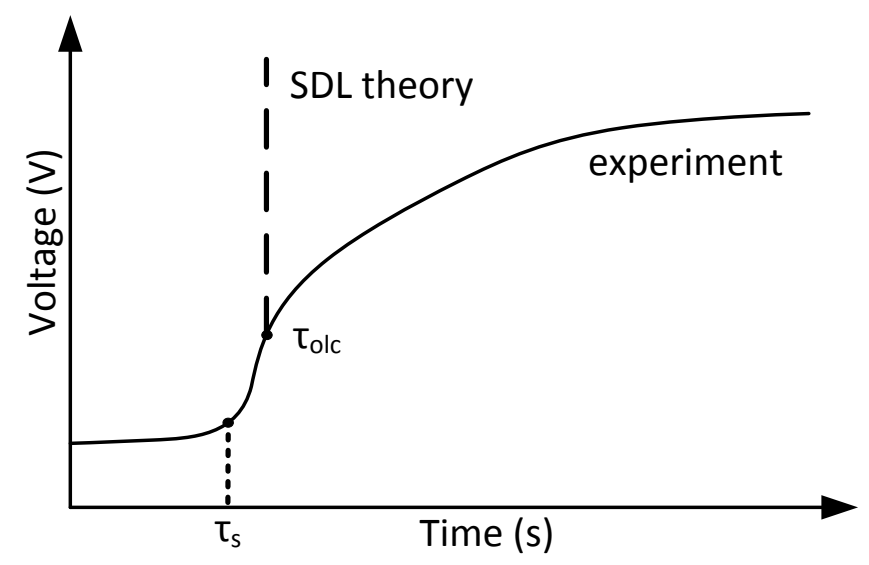

Figure 1.9: Typical voltage response after applying a constant current through a membrane.

towards the membrane where it depletes and flows back. The one dimensional description of the concentration profile therefore breaks down. The advection can either be driven by the electric field, called electroconvection (section 1.7.1) or by the gravitational field (section 1.7.2).

The second group ascribes OLC to chemical processes. For a long time water dissociation at the membrane interface was assumed to be the cause of OLC $[40,57-59]$. At a critical voltage water can dissociate into protons and hydroxyl ions which serve as additional charge carriers with a high mobility, thus causing an increase in current above a threshold.

$$
2 \mathrm{H}_{2} \mathrm{O} \rightleftharpoons \mathrm{H}_{3} \mathrm{O}^{+}+\mathrm{OH}^{-}
$$

Changes in $\mathrm{pH}$ of the treated solution are evidence that water dissociation can occur. Further investigation has shown that this mostly occurs at anion exchange membranes, not at cation exchange membranes, due to the difference in active ionic groups [35, 60, 61]. Many researchers found that water dissociation at cation exchange membranes during overlimiting current conditions is negligible regarding their contribution to OLC [35, 62, 63].

There is also another chemical theory that describes the loss of the membrane selectivity at a threshold voltage [64]. This theory states that at the depleted side the increased electric field could induced a $\mathrm{pH}$ shift in the membrane, thereby reducing its charge selectivity. This remains an unconfirmed theory and many experimentalist have not measured noticeable changes in membrane permselectivity $[35,61,65,66]$. 


\subsubsection{Electroconvection}

An electric potential gradient in an electrolyte causes ion migration, which can lead to fluid motion, as can be seen from the coupling between the NernstPlanck, Poisson and Navier-Stokes equation in section 1.3. In an electroneutral electrolyte the anions and cations have an opposite charge and are forced in opposite directions. Ions in movement collide with the neutral water molecules and part of the momentum is transferred. In general the momentum transfer from the anions and cations is similar and cancel each other out such that the water stays stagnant [67]. Generally the loss of kinetic energy dissipates as heat.

In case the electrolyte is not electroneutral, a net force can be exerted on the water molecules causing fluid motion, therefore called electrohydrodynamics, or electroconvection. Typically this happens at the solid-liquid interface. There an electric double layer (EDL) forms consisting of the fixed charges in the solid interface and a layer of mobile charges in the liquid phase. When the electric force sets the water in motion this is called electro-osmosis. When the solid itself, for example small particles, is set in motion one speaks of electrophoresis. The opposite effect is also possible, the emergence of an electric potential due to the motion of the liquid or solid. For more information in this topic one can read $[10,38]$.

Electro-osmosis happens when the electric field has a parallel component to the surface. In electrodialysis the field is applied perpendicular to the membrane surface. However still electrically induced mixing in the depleted layer has been observed [68-70]. The charge in the interface layer can be unevenly distributed along the membrane because of fluctuations in surface geometry or chemistry $[7,71]$, concentration, temperature or pressure $[37,72,73]$. This can lead to an electric field along the membrane $\left(E_{y} \neq 0\right)$ and is described by Poisson's equation 1.10. This lateral electric field in the charged layer can exert a net force on the liquid. A large amount of theoretical and numerical work has been conducted to investigate when this force is strong enough to cause mixing perpendicular to the applied electric field $[4,37,72,74-76]$ and starts with a threshold voltage as is typically seen (figure 1.4).

Most theories are based on the extension of the non-electroneutral double layer at the membrane surface due to the concentration depletion. This layer is referred to as the extended space charge layer (ESCL). In the ESCL the electro-osmotic force transfer is stronger since the local friction is less compared to closer to the membrane wall. In case the surface is curved with respect to the electric field, the field will have a normal and a tangential component. This tangential component can drive the electro-osmosis. In literature this is referred to as the Dukhin mechanism or induced charge electro-osmosis (ICEO) [74, 76-78]. 
Rubinstein and Zaltzman showed that the ESCL can also drive tangential electro-osmosic at a flat surface [4]. Once an inhomogeneity, e.g. from a locally enriched concentration in the ESCL, causes lateral motion (see figure 1.10), the pressure at that spot drops $(\Delta P)$. This will allow water to flow as can be seen from the Navier-Stokes equation 1.11. The flow directed to the membrane will have higher concentration, therefore enhancing the initial concentration enrichment and electro-osmotic vortex motion. This positive feedback mechanism is referred to as the Rubinstein mechanism or the electrokinetic instability (EKI) $[37,76]$. It is called an instability since the feedback mechanism only works above a certain threshold voltage, needed to overcome the natural resistances.

A combination of these two mode of electroconvection has been predicted to enhance the current efficiency once the membrane undulation has the same order as the mixing layer thickness $[4,76]$. Experimental work has indeed shown a reduction in resistance when structures on the membrane were present, either geometrical [7] or chemical [71].

a)

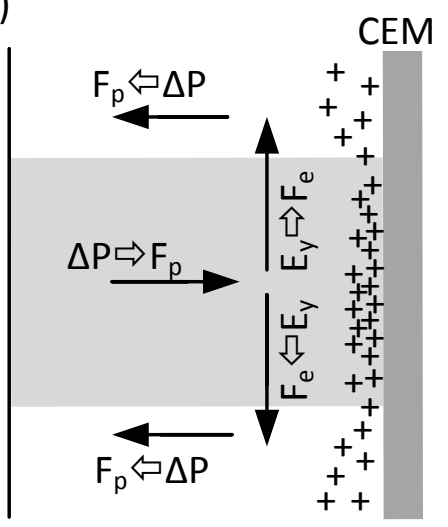

b)

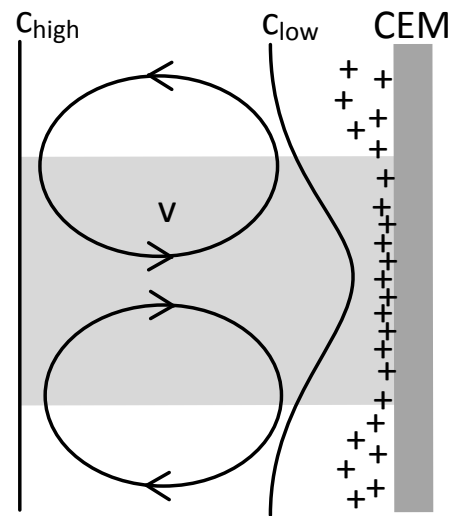

Figure 1.10: A schematic of the coupling between the electric field and fluid. A higher interface concentration at the middle of the picture (gray area) causes a thinned but higher charged electric space charge layer. This results in a lateral electric gradient $\left(E_{\mathrm{y}}\right)$ and electrical body force $\left(F_{\mathrm{e}}\right)$ outwards towards the white region. This in turn causes pressure gradients which start the vortical motion which brings higher concentration liquid to the interface in the gray area and enhances the initial inhomogeneity.

\subsubsection{Gravitational convection}

Within the fluid the local density can vary due to concentration or temperature gradients. If the gradient is perpendicular to the gravitational field, convection 
will always start and this is known as natural convection [79]. If the gradient is along the gravitational field direction, Rayleigh-Bénard (RB) convection occurs if the lighter (ion depleted) fluid lies below a heavier (ion enriched) fluid and a critical gradient has been reached for the buoyancy forces to overcome the viscous forces. Their ratio can be expressed by the dimensionless Rayleigh number [80]:

$$
R a=\frac{M \Delta c g L^{3}}{\mu D}
$$

where $M[\mathrm{~kg} / \mathrm{mol}]$ is the molar mass of the electrolyte, $\Delta c\left[\mathrm{mM}=\mathrm{mol} / \mathrm{m}^{3}\right]$ is the characteristic concentration difference, $g=9.81 \mathrm{~m} / \mathrm{s}^{2}$ is the gravitational constant, $L[\mathrm{~m}]$ is the characteristic length scale of the gradient, $\mu=$ $1.002 \cdot 10^{-9} \mathrm{~kg} / \mathrm{m} / \mathrm{s}$ is the dynamic viscosity, and $D$ is the diffusion coefficient of the electrolyte. To be precise the Rayleigh number is the multiplication of the Grashof number and the Schmidt number [81]:

$$
R a=G r \cdot S c=\frac{\text { buoyancy forces }}{\text { viscous forces }} \cdot \frac{\text { momentum diffusivity }}{\text { mass diffusivity }}
$$

It was found by Chandrasekhar that if $R a>1708$ a gradient between two rigid boundaries becomes unstable [79]. For systems with one rigid and one stress free boundary the instability starts at $R a>1101$ [79]. Similar values have been found through experimental work [82-84].

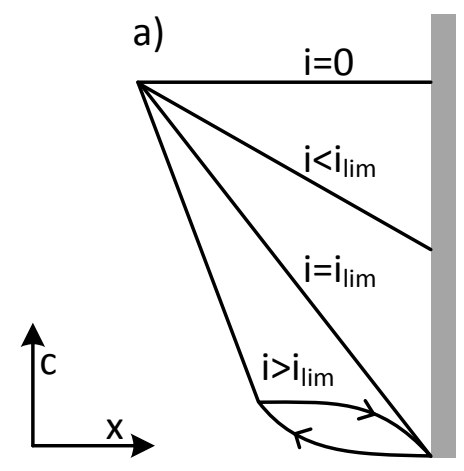

b)

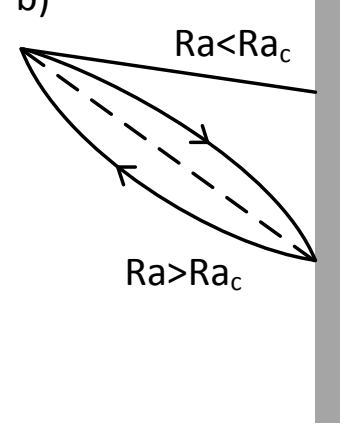

Figure 1.11: Schematic of the concentration profile near the membrane with an electroconvective mixing layer (a) and an gravitational driven mixing layer (b). The line with arrows indicate the vortical motion with high concentrated solution towards the membrane and low concentrated solution away from the membrane. The dashed line shows the initial unstable concentration gradient.

Gravitational convection occurs over the full gradient, while electroconvection only at the membrane interface, see figure 1.11. This difference is explored in the 
visual investigation of convection at an ion exchange membrane.

\subsection{Scope of thesis}

In the previous sections the theoretical basis behind this work is discussed. As explained, overlimiting current is of rising interest to fundamental science as well as to engineering. The enhanced current transport per membrane surface area could improve, for example, the cost effectiveness of electrodialysis systems. The idea of more efficient electroconvective mixing at a structured membrane is worth pursuing. The research presented in this thesis puts great interest in the observation and description of the overlimiting current regime. Moreover, further research is conducted on the study and determination of the performance of an overlimiting current applied across flat and structured membranes.

In Chapter 2, an overview of the experimental methods and their backgrounds used in this thesis are described. The electrochemical method to induce overlimiting current at a cation ion exchange membrane is explained. From the time dependent electrical signal the different current regimes, ohmic, limiting and overlimiting, can be distinguished. Simultaneously optical measurements are conducted to deduce the changing flow or concentration at the depleted side of the membranes. Either a 2D flow velocity field is extracted using particle image velocimetry (PIV), or a $2 \mathrm{D}$ concentration image is determined using fluorescent lifetime image microscopy (FLIM).

In Chapter 3, overlimiting current at a flat membrane due to electroconvection is quantified. A constant current is applied across the membrane and the time-dependent resistance and flow dynamics (PIV) are observed simultaneously. Initially the system displays a steady Ohmic voltage difference $\left(\Delta V_{\text {ohm }}\right)$. The time of depletion is in agreement with theory. Immediately after this depletion time, electroconvective micro-vortices near the depleted side of the membrane set in and grow both in size and speed with time. After this growth, the resultant voltage levels off around a fixed value. The average vortex size and speed stabilize as well, while the individual vortices become unsteady and dynamic. These quantitative results reveal that micro-vortices set in with an excess voltage drop (above $\Delta V_{o h m}+\Delta V_{c}$ ) and sustain an approximately constant electrical conductivity, destroying the initial ICP.

In Chapter 4, the interaction between gravitational convection and electroconvection is investigated. In the counter-orientation (as in Chapter 3) only electroconvection drives OLC. In the co-orientation (reversed orientation compared to the gravitational field) also Rayleigh-Bénard convection occurs which mixes the full compartment and reduces the resistance. The properties of both 
types of convection is determined with electrical, flow (PIV) and concentration (FLIM) measurements. The onset of both instabilities was also predicted with Fick's laws of diffusion. The experimental and numerical data on the onset of electroconvection and RB convection are in agreement.

In Chapter 5, membranes with a designed geometrical structure are shown to enhance the overlimiting ion transport due to electroconvection. Membranes with square wave structures with different periodicity were synthesized. The membrane was placed in the test cell where the flow (PIV), concentration (FLIM) and resistance (constant voltage) were determined. The electroconvective vortex shape stabilizes when the mixing layer height is similar to the structure width. The stable vortices have lower resistance compared to lateral moving vortices that occur at flat membranes. The combination of the quantitative electrical, flow and concentration measurements allow an estimation of the migration, advection and diffusion of ions as described by the Nerst-Planck equation. These estimates confirm that in the mixing layer migration and advection are the dominant ion transport mechanisms in contrast to migration and diffusion on the stagnant layer.

In Chapter 6, a summary out of the work in this thesis is presented as well as an outlook for future experiments and applications. Several ideas for other surface structures as well as chemical membrane structures are discussed. The relation between experimental and numerical investigation of OLC and its striking similarities and differences are reviewed and ideas are presented to bridge the cap between both worlds. Finally the application of overlimiting current in electrodialysis operation is discussed along with a concept in which OLC reduces the resistance to near Ohmic properties. 



\section{Bibliography}

[1] D. E. Lide, CRC Handbook of Chemistry and Physics, 76th ed. (CRC Press, 1996), pp. 5-90. - p.11, 13, 17, 21.

[2] H. Strathmann, Ion exchange membrane separation processes - membrane science and technology series 9, 1st editio ed. (Elsevier, 2004), No. 9. p.11, 12, 14, 15, 18.

[3] V. V. Nikonenko, A. V. Kovalenko, M. K. Urtenov, N. D. Pismenskaya, J. Han, P. Sistat, and G. Pourcelly, Desalination at overlimiting currents: State-of-the-art and perspectives, Desalination 342, 85 (2014). — p.12, 18, 27.

[4] I. Rubinstein and B. Zaltzman, Electro-osmotically induced convection at a permselective membrane, Physical Review E 62, 2238 (2000). — p.12, 13, 29,30 .

[5] J. H. Choi, J. S. Park, and S. H. Moon, Direct measurement of concentration distribution within the boundary layer of an ion-exchange membrane, , Journal of Colloid and Interface Science 251, 311 (2002). — p..

[6] C. Druzgalski and A. Mani, Statistical analysis of electroconvection near an ion-selective membrane in the highly chaotic regime, Physical Review Fluids 1, 073601 (2016). - p.12.

[7] J. Balster, M. H. Yildirim, D. F. Stamatialis, R. Ibanez, R. G. H. Lammertink, V. Jordan, and M. Wessling, Morphology and microtopology of cation-exchange polymers and the origin of the overlimiting current, The journal of physical chemistry. B 111, 2152 (2007). — p.13, 29, 30.

[8] M. Mulder, in Basic Principles of Membrane Technology, edited by 2nd (Kluwer Academic Publishers, Dordrecht, 1996), p. 564. — p.13, 14, 18, 21.

[9] A. Casadellà, O. Schaetzle, and K. Loos, Ammonium across a Selective Polymer Inclusion Membrane: Characterization, Transport, and Selectivity, Macromolecular Rapid Communications 37, 858 (2016). — p.13.

[10] R. F. Probstein, Physicochemical hydrodynamics : an introduction (Wiley, 1994), p. 400. - p.13, 18, 19, 20, 26, 29.

[11] T. Xu, Ion exchange membranes: State of their development and perspective, Journal of Membrane Science 263, 1 (2005). - p.14. 
[12] J. Ran, L. Wu, Y. He, Z. Yang, Y. Wang, C. Jiang, L. Ge, E. Bakangura, and T. Xu, Ion Exchange Membranes: New Developments and Applications, Journal of Membrane Science 522, 267 (2016). — p.14.

[13] S. Porada, R. Zhao, A. Van Der Wal, V. Presser, and P. M. Biesheuvel, Review on the science and technology of water desalination by capacitive deionization, Progress in Materials Science 58, 1388 (2013). — p.14.

[14] H. Strathmann, Electrodialysis, a mature technology with a multitude of new applications, Desalination 264, 268 (2010). — p.14, 17, 18.

[15] J. Veerman, M. Saakes, S. J. Metz, and G. J. Harmsen, Reverse electrodialysis: Performance of a stack with 50 cells on the mixing of sea and river water, Journal of Membrane Science 327, 136 (2009). — p.14, 15.

[16] D. A. Vermaas, M. Saakes, and K. Nijmeijer, Power generation using profiled membranes in reverse electrodialysis, Journal of Membrane Science 385-386, 234 (2011). - p.14.

[17] G. Merle, M. Wessling, and K. Nijmeijer, Anion exchange membranes for alkaline fuel cells: A review, Journal of Membrane Science 377, 1 (2011). - p.14.

[18] S. J. Peighambardoust, S. Rowshanzamir, and M. Amjadi, International journal of hydrogen energy (Elsevier Ltd, 2010), No. 17, pp. 9349-9384. p.14.

[19] S. D. Molenaar, A. R. Mol, T. H. J. A. Sleutels, A. Ter Heijne, and C. J. N. Buisman, Microbial Rechargeable Battery: Energy Storage and Recovery through Acetate, Environmental Science \& Technology Letters 3, 144 (2016). - p.14.

[20] Y. Tanaka, Ion exchange membranes fundamentals and applications, 1st ed. (Elsevier, 2007), p. 546. - p.14.

[21] S. E. Kentish, E. Kloester, G. W. Stevens, C. a. Scholes, and L. F. Dumée, Electrodialysis in Aqueous-Organic Mixtures, Separation \& Purification Reviews 44, 269 (2014). - p.18.

[22] C. Larchet, V. I. Zabolotsky, N. Pismenskaya, V. V. Nikonenko, A. Tskhay, K. Tastanov, and G. Pourcelly, Comparison of different ED stack conceptions when applied for drinking water production from brackish waters, Desalination 222, 489 (2008). - p.14, 24.

[23] J. Veerman, M. Saakes, S. J. Metz, and G. J. Harmsen, Reverse electrodialysis: Evaluation of suitable electrode systems, Journal of Applied Electrochemistry 40, 1461 (2010). - p.14.

[24] A. H. Galama, J. W. Post, H. V. M. Hamelers, V. V. Nikonenko, and P. M. Biesheuvel, On the origin of the membrane potential arising across densely charged ion exchange membranes : How well does the Teorell-Meyer-Sievers theory work ?, Journal of Membrane Science and Research 2, 128 (2015). 
- p.15.

[25] P. Długołęcki, P. Ogonowski, S. J. Metz, M. Saakes, K. Nijmeijer, and M. Wessling, On the resistances of membrane, diffusion boundary layer and double layer in ion exchange membrane transport, Journal of Membrane Science 349, 369 (2010). — p.15, 20.

[26] D. J. Griffiths, Introduction to electrodynamics, 3th ed. (Prentice-Hall International, Inc., New Jersey, 1999), p. 576. - p.15.

[27] M. Elimelech and W. A. Phillip, The future of seawater desalination: energy, technology, and the environment, Science (New York, N.Y.) 333, 712 (2011). - p.18.

[28] M. Fidaleo, M. Moresi, A. Cammaroto, N. Ladrange, and R. Nardi, Soy sauce desalting by electrodialysis, Journal of Food Engineering 110, 175 (2012). - p.18.

[29] S. Abdu, M.-C. Martí-Calatayud, J. E. Wong, M. García-Gabaldón, and M. Wessling, Layer-by-layer modification of cation exchange membranes controls ion selectivity and water splitting, ACS applied materials \& interfaces 6, 1843 (2014). - p.18.

[30] A. H. Galama, G. Daubaras, O. S. Burheim, H. H. M. Rijnaarts, and J. W. Post, Seawater electrodialysis with preferential removal of divalent ions, Journal of Membrane Science 452, 219 (2014). — p.18.

[31] B. Pilat, Practice of water desalination by electrodialysis, Desalination 139, 385 (2001). - p.18.

[32] S. Mikhaylin and L. Bazinet, Fouling on ion-exchange membranes: Classification, characterization and strategies of prevention and control, Advances in Colloid and Interface Science 229, 34 (2016). - p.18.

[33] J. W. Post, H. Huiting, E. R. Cornelissen, and H. V. M. Hamelers, Predesalination with electro-membranes for SWRO, Desalination and Water Treatment 31, 296 (2011). - p.18.

[34] R. S. L. Yee, R. A. Rozendal, K. Zhang, and B. P. Ladewig, Cost effective cation exchange membranes: A review, Chemical Engineering Research and Design 90, 950 (2012). - p.18.

[35] J. J. Krol, M. Wessling, and H. Strathmann, Concentration polarization with monopolar ion exchange membranes: current-voltage curves and water dissociation, Journal of Membrane Science 162, 145 (1999). — p.18, 28.

[36] J. Wesselingh and R. Krishna, Mass transfer in multicomponent mixtures, 1st ed. ed. (Delft University Press, Delft Netherland, 2000), p. 329. — p.19.

[37] C. L. Druzgalski, M. B. Andersen, and a. Mani, Direct numerical simulation of electroconvective instability and hydrodynamic chaos near an ion-selective surface, Physics of Fluids 25, 110804 (2013). - p.19, 20, 29, 30.

[38] H. Bruus, Theoretical microfluidics (Oxford University Press, 2008). 
p.20, 24, 29.

[39] E. A. Demekhin, S. Amiroudine, G. S. Ganchenko, and N. Y. Khasmatulina, Thermoelectroconvection near charge-selective surfaces, Physical Review E Statistical, Nonlinear, and Soft Matter Physics 91, 1 (2015). — p.20.

[40] V. V. Nikonenko, N. D. Pismenskaya, E. I. Belova, P. Sistat, P. Huguet, G. Pourcelly, and C. Larchet, Intensive current transfer in membrane systems: Modelling, mechanisms and application in electrodialysis, Advances in Colloid and Interface Science 160, 101 (2010). - p.22, 28.

[41] M. Tedesco, H. V. M. Hamelers, and P. M. Biesheuvel, Nernst-Planck transport theory for (reverse) electrodialysis: I. Effect of co-ion transport through the membranes, Journal of Membrane Science 510, 370 (2016). — p.22.

[42] S. Pawlowski, P. Sistat, J. G. Crespo, and S. Velizarov, Mass transfer in reverse electrodialysis: Flow entrance effects and diffusion boundary layer thickness, Journal of Membrane Science 471, 72 (2014). — p.23.

[43] R. Kwak, G. Guan, W. K. Peng, and J. Han, Microscale electrodialysis: Concentration profiling and vortex visualization, Desalination 308, 138 (2013). - p.23.

[44] A. A. Sonin and M. S. Isaacson, Optimization of flow design in forced flow electrochemical systems, with special application to electrodialysis, Industrial and Engineering Chemistry: Process and Design Development 13, 241 (1974). - p.23, 24.

[45] B. Kim, S. Choi, V. S. Pham, R. Kwak, and J. Han, Energy efficiency enhancement of electromembrane desalination systems by local flow redistribution optimized for the asymmetry of cation/anion diffusivity, Journal of Membrane Science 524, 280 (2017). — p.23.

[46] M. S. Isaacson and A. a. Sonin, Sherwood Number and Friction Factor Correlations for Electrodialysis Systems, with Application to Process Optimization, Industrial \& Engineering Chemistry Process Design and Development 15, 313 (1976). - p.24.

[47] D. H. Kim, I. H. Kim, and H. N. Chang, Experimental study of mass transfer around a turbulence promoter by the limiting current method, International Journal of Heat and Mass Transfer 26, 1007 (1983). — p.24.

[48] A. M. Peers, No Title, Discuss.Faraday Soc. 21 (1956). — p.25.

[49] E. Kreyszig, Advanced Engineering Mathematics, 9th ed. (Wiley, 2005). p.26.

[50] A. J. Bard and L. R. Faulkner, Methods, 2nd ed. (2001), pp. xxi, 833 p. - p.26, 27.

[51] P. Sistat and G. Pourcelly, Chronopotentiometric response of an ionexchange membrane in the underlimiting current-range. Transport phenomena within the diffusion layers, Journal of Membrane Science 123, 121 
(1997). - p.26.

[52] H. J. Sand, On the concentration at the electrodes in a solution, with special reference to the liberation of hydrogen by electrolysis of a mixture of copper sulphate and sulphuric acid, Philosophical Magazine Series 6 1, 45 (1901). - p.27.

[53] S. Mareev, D. Butylskii, A. Kovalenko, A. Petukhova, N. Pismenskaya, L. Dammak, C. Larchet, and V. Nikonenko, Accounting for the concentration dependence of electrolyte diffusion coefficient in the Sand and the Peers equations, Electrochimica Acta 195, 85 (2016). — p.27.

[54] M. Van Soestbergen, P. M. Biesheuvel, and M. Z. Bazant, Diffuse-charge effects on the transient response of electrochemical cells, Physical Review E - Statistical, Nonlinear, and Soft Matter Physics 81, 1 (2010). — p.27.

[55] J. Krol, M. Wessling, and H. Strathmann, Chronopotentiometry and overlimiting ion transport through monopolar ion exchange membranes, Journal of membrane science 162, 155 (1999). - p.27.

[56] F. Maletzkl, H. Rdsler, E. Staude, F. Maletzki, H.-W. Rösler, and E. Staude, Ion transfer across electrodialysis membranes in the overlimiting current range : stationary voltage current characteristics and current noise power spectra under different conditions of free convection, Journal of Membrane Science 71, 105 (1992). - p.27.

[57] C. Forgacs, N. Ishibashi, J. Leibovitz, J. Sinkovic, and K. S. Spiegler, Polarization at ion-exchange membranes in electrodialysis, Desalination 10, 181 (1972). - p.28.

[58] R. Simons, The origin and elimination of water splitting in ion exchange membranes during water demineralisation by electrodialysis, Desalination 28, 41 (1979). - p..

[59] V. I. Zabolotsky, V. V. Nikonenko, N. D. Pismenskaya, E. V. Laktionov, M. K. Urtenov, H. Strathmann, M. Wessling, and G. H. Koops, Coupled transport phenomena in overlimiting current electrodialysis, Separation and Purification Technology 14, 255 (1998). — p.28.

[60] Y. Tanaka, Water dissociation reaction generated in an ion exchange membrane, Journal of Membrane Science 350, 347 (2010). — p.28.

[61] M. Taky, G. Pourcelly, and C. Gavach, Polarization phenomena at the interfaces between an electrolyte solution and an ion exchange membrane. Part II. Ion transfer with an anion exchange membrane, Journal of Electroanalytical Chemistry 336, 195 (1992). - p.28.

[62] M.-S. Kang, Y.-J. Choi, and S.-H. Moon, Effects of charge density on water splitting at cation-exchange membrane surface in the over-limiting current region, Korean Journal of Chemical Engineering 21, 221 (2004). — p.28.

[63] I. Rubinstein and L. Shtilman, Voltage against current curves of cation ex- 
change membranes, Journal of the Chemical Society, Faraday Transactions 2 75, 231 (1979). - p.28.

[64] M. B. Andersen, M. Van Soestbergen, a. Mani, H. Bruus, P. M. Biesheuvel, and M. Z. Bazant, Current-induced membrane discharge, Physical Review Letters 109, 1 (2012). - p.28.

[65] M. Taky, G. Pourcelly, F. Lebon, and C. Gavach, Polarization phenomena at the interfaces between an electrolyte solution and an ion exchange membrane Part I . Ion transfer with a cation exchange membrane, 336, 171 (1992). p.28.

[66] M. Block and J. a. Kitchener, Polarization Phenomena in Commercial IonExchange Membranes, Journal of The Electrochemical Society 113, 947 (1966). - p.28.

[67] H. C. Chang, E. A. Demekhin, and V. S. Shelistov, Competition between Dukhin's and Rubinstein's electrokinetic modes, Physical Review E 86, (2012). - p.29.

[68] S. Rubinstein, G. Manukyan, A. Staicu, I. Rubinstein, B. Zaltzman, R. Lammertink, F. Mugele, and M. Wessling, Direct Observation of a Nonequilibrium Electro-Osmotic Instability, Physical Review Letters 101, (2008). - p.29.

[69] G. Yossifon and H.-C. Chang, Selection of Nonequilibrium Overlimiting Currents: Universal Depletion Layer Formation Dynamics and Vortex Instability, Physical Review Letters 101, 254501 (2008). — p..

[70] S. Kim, Y.-C. Wang, J. Lee, H. Jang, and J. Han, Concentration Polarization and Nonlinear Electrokinetic Flow near a Nanofluidic Channel, Physical Review Letters 99, 044501 (2007). — p.29.

[71] M. Wessling, L. G. Morcillo, and S. Abdu, Nanometer-thick lateral polyelectrolyte micropatterns induce macrosopic electro-osmotic chaotic fluid instabilities., Scientific reports 4, 4294 (2014). — p.29, 30.

[72] A. S. Khair, Concentration polarization and second-kind electrokinetic instability at an ion-selective surface admitting normal flow, Physics of Fluids 23, 072003 (2011). - p.29.

[73] J. A. Wood, A. M. Benneker, and R. G. Lammertink, Temperature effects on the electrohydrodynamic and electrokinetic behaviour of ion-selective nanochannels, Journal of Physics: Condensed Matter 28, 114002 (2016). p.29.

[74] S. S. Dukhin, Electrokinetic phenomena of the second kind and their applications, Advances in Colloid and Interface Science 35, 173 (1991). — p.29.

[75] V. S. Pham, Z. Li, K. M. Lim, J. K. White, and J. Han, Direct numerical simulation of electroconvective instability and hysteretic current-voltage response of a permselective membrane, Physical Review E 86, (2012). — p.. 
[76] E. a. Demekhin, N. V. Nikitin, and V. S. Shelistov, Direct numerical simulation of electrokinetic instability and transition to chaotic motion, Physics of Fluids 25, 122001 (2013). — p.29, 30.

[77] N. a. Mishchuk, Concentration polarization of interface and non-linear electrokinetic phenomena., Advances in colloid and interface science 160, 16 (2010). - p..

[78] M. Z. Bazant, M. S. Kilic, B. D. Storey, and A. Ajdari, Towards an understanding of induced-charge electrokinetics at large applied voltages in concentrated solutions., Advances in colloid and interface science 152, 48 (2009). - p.29.

[79] E. Cussler, Diffusion, mass transfer in fluid systems (Cambridge University Press, 1984), p. 580. - p.31.

[80] W. J. Ward and O. H. L. Blanc, Rayleigh-Benard Convection in an Electrochemical Redox Cell, No. 4669 in New Series, Science 225, 1471 (1984). - p.31.

[81] V. I. Zabolotsky, V. V. Nikonenko, and N. D. Pismenskaya, On the role of gravitational convection in the transfer enhancement of salt ions in the course of dilute solution electrodialysis, Journal of Membrane Science 119, 171 (1996). - p.31.

[82] B. Baranowski and A. Kawczyński, Experimental determination of the critical rayleigh number in electrolyte solutions with concentration polarization, Electrochimica Acta 17, 695 (1972). - p.31.

[83] E. E. Mocskos, G. González, F. V. Molina, and G. Marshall, Numerical and experimental studies of Electrochemical Deposition quasi-stable growth, Journal of Electroanalytical Chemistry 653, 27 (2011). — p..

[84] E. Hage and a. Tilgner, High Rayleigh number convection with double diffusive fingers, Physics of Fluids 22, 1 (2010). - p.31. 



\section{CHAPTER 2}

\section{Experimental methods}

This chapter describes the experimental methods, along with their theoretical backgrounds used in this thesis to study the ion transport in the boundary layer near an ion exchange membrane (IEM). In particular the methods to measure the influence of electroconvection and gravitational convection on the ion transport during overlimiting current (OLC). To determine the migration, advection and diffusion terms in the Nernst-Planck equation (eq. 1.14) a combination of electrochemical, flow and concentration measurements are carried out. By a controlled electrical potential difference (or current) ion concentration polarization (ICP) is induced and the time dependent resistance is monitored in an electrochemical cell. The cell is transparent to visualize the flow motion induced by the convection in the boundary layer via particle tracking and particle image velocimetry (PIV). Alternatively, a novel approach based on fluorescent lifetime microscopy (FLIM) is used to determine the ion concentration distribution in the boundary layer

\subsection{Electrochemical measurements}

Electrochemical measurements are used to: a) induce the ion migration which causes the ion concentration polarization (ICP) near the membrane surface, b) measure the time-dependent resistance in the system. The electrolyte resistance depends on the ion concentration (see eq. 1.6). It is therefore an indicator of the state of the concentration polarization and of the power needed to transport ions through the system.

The relation between the applied voltage and the resulting current has been an essential tool to understand ICP and to design electrodialysis systems [1]. The sharp increase in resistance due to the ion depletion (the limiting current) is an important operational parameter to understand the concentration polarization inside the stack and find the optimal operational conditions [2-4]. When advective mixing in the channels is enhanced due to obstacles the limiting current increased. The trade-off between pressure loss, electrical resistance, product 
quality and system costs determines the proper operational settings.

Overlimiting current (OLC) is widely observed and can result from numerous mechanisms and is system specific, as discussed in section 1.7. The multitude of phenomena, such as water splitting, electroconvection, gravitational convection and pressure driven convection [5] called for more controlled experimental systems. This led to the development of an electrodialysis cell with reference electrodes placed on both sides of a membrane. Together with the mass balance and flow rate more information could be obtained.

The electrical measurements are preformed by placing a controlled voltage difference between the anode and cathode using a potentiostat. The anode is connected to the working electrode (WE) and gets positively charged. The cathode is connected to the counter electrode (CE) and get negatively charged. Two other electrodes are used to measure the voltage or current, the sense electrode $(\mathrm{SE})$ and reference electrode $(\mathrm{RE})$. Sometimes additional reference electrode are used to gain more information on the voltage drop in the system.

The redox reactions at the electrodes transfer the electric current into ionic current [6]. These reactions depend on the electrode material and electrolyte ion content. In early research the membrane was clamped between two compartments containing the electrolyte solution [7-9]. This approach presented drawbacks since the steady state could not be reached. Later, an approach using a system with 6 compartments to buffer the electrode effects was used [10, 11]. However it also presented drawbacks since the mass balance was difficult to determine (i.e. various electrolyte solutions are used to run the system, the membrane transport number cannot be easily calculated). An approach to overcome these drawbacks consists of a two compartment cell containing $\mathrm{CuSO}_{4}$ solutions as electrolytes and copper electrodes. The fact that both electrolytes and electrodes are based on copper makes a steady state reachable and the mass balance is easily related to the applied current since no other ions are present [12-15]. This thesis includes a further improvement to the latter approach by adding two copper wire electrodes on both side of the membrane that can be used as sense and reference electrode.

The dominant redox reactions at the electrodes are [16]:

$$
\mathrm{Cu}_{0}(\mathrm{~s}) \rightleftharpoons \mathrm{Cu}^{2+}(\mathrm{aq})+2 \mathrm{e}^{-} \quad \Delta V=0.34 \mathrm{~V} \text { vs. SHE }
$$

An overpotential of $0.34 \mathrm{~V}$ is needed to release copper ions from the copper anode into the electrolyte. At the cathode, with an ideal overpotential of $-0.34 \mathrm{~V}$, copper ions are deposited on the copper surface. The electrodeposition process is intensively studied and used for many application like protective coatings or active layers [17-19]. 
a)

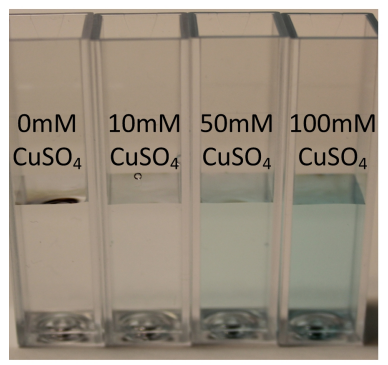

b)

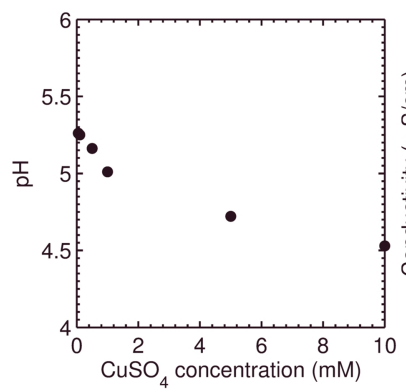

c)

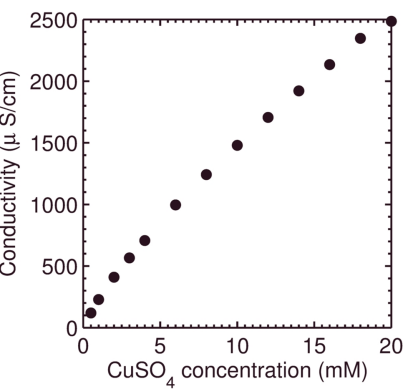

Figure 2.1: a) The color $\mathrm{CuSO}_{4}$ electrolyte depends on the concentration 1:0mM, 2:10mM, 3:50mM 4:100mM. b) The $\mathrm{pH}$ decreases with increasing the $\mathrm{CuSO}_{4}$ concentration. c) Calibration curve of $\mathrm{CuSO}_{4}$ concentration vs conductivity.

\subsection{1 $\mathrm{CuSO}_{4}$ properties}

Most measurements are conducted with a $10 \mathrm{mM} \mathrm{CuSO}_{4}$ electrolyte solution. This is made by the dissolving $\mathrm{CuSO}_{4} \cdot 5 \mathrm{H}_{2} \mathrm{O}[250 \mathrm{~g} / \mathrm{mol}]$ (VWR Chemicals) in deionized water (milliQ). The salt dissociates into two divalent ions: $\mathrm{SO}_{4}{ }^{2-}$ and $\mathrm{Cu}^{2+}$. Part of the $\mathrm{Cu}^{2+}$ cations form a complex with $\mathrm{OH}^{-}$, this shifts the equilibrium of water dissociation, leading to an effective higher concentration of $\mathrm{H}^{+}$, decreasing the $\mathrm{pH}[16]$. Because the solution is in contact with air, it absorbs $\mathrm{CO}_{2}$ from the atmosphere leading to the presence of carbonic acid $\left(\mathrm{H}_{2} \mathrm{CO}_{3} \rightleftharpoons\right.$ $\mathrm{H}^{+}+\mathrm{HCO}_{3}{ }^{-}$), decreasing the $\mathrm{pH}$ further. The $\mathrm{pH}$ is measured to be around 4.5 for a $10 \mathrm{mM}$ solution, see figure 2.1b. The concentration of $\mathrm{H}^{+}, \mathrm{OH}^{-}$and $\mathrm{HCO}_{3}{ }^{-}$ is negligible compared to the $\mathrm{Cu}^{2+}$ and $\mathrm{SO}_{4}{ }^{2-}$ concentration. The current can therefore be primarily connected to these two divalent ions.

The conductivity of the electrolyte is dependent on concentration, see figure 2.1c. At $10 \mathrm{mM}$ the conductivity is $\sigma \approx 1.5 \mathrm{mS} / \mathrm{cm}$. This is in the order of river water $\sigma \approx 0.7 \mathrm{mS} / \mathrm{cm}$. The total dissolved salt at $10 \mathrm{mM} \mathrm{CuSO}_{4}$ is $2.5 \mathrm{~g} / \mathrm{L}$, which is in the order of brackish water $(1-5 \mathrm{~g} / \mathrm{L})$. This is also the range in which ED possesses an economic advantage over reverse osmosis [20, 21].

\subsubsection{Membrane test cell}

The membrane is placed in a transparent polymethyl methacrylate (PMMA) cell designed be able to visualize the flow in the membrane boundary layer during overlimiting current conditions. The cell is filled with a $\mathrm{CuSO}_{4}$ electrolyte between two copper electrodes, see figure 2.2. The cell is constructed from three 
blocks. The middle block has a small opening to place the membrane for it to separate the two reservoirs. The opening consists of a semi-circle of $10 \mathrm{~mm}$ with a height of $\approx 0.2 \mathrm{~mm}$. The open surface area of the membrane is equal to anode area and is $3 \mathrm{~mm} \times 4.5 \mathrm{~mm}\left(A_{\text {mem }}=13.5 \mathrm{~mm}^{2}\right)$. They are placed $2 \mathrm{~mm}$ from each other. In this reservoir the electrolyte will deplete next to the membrane and enrich next to the anode.

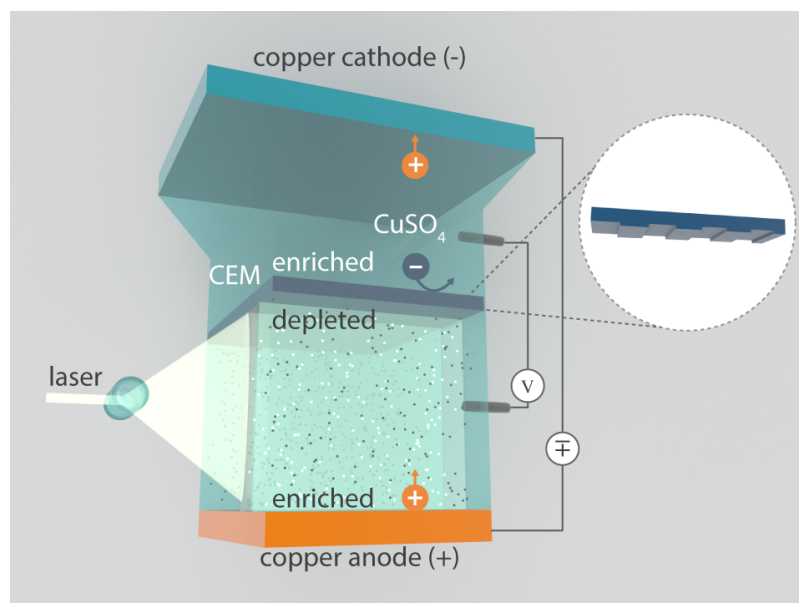

Figure 2.2: Schematic of the copper electrode membrane test cell. Next to the electrical measurements the flow is visualized by tracer particles. Different membranes are investigated.

An optical window seals the channel from the front. It is made from borosilicate based glass (BK7) which has an index of refraction of $n=1.5$ (CRYSTRAN Ltd). The dimensions of the window are $15 \times 15 \times 1 \mathrm{~mm}^{3}$. The window is glued into an aluminium frame that is screwed to the cell with an O-ring behind it (figure 2.3). A second optical window is placed on the left side of the anode compartment to allow the entry of a laser light. The dimensions of this second BK7 window are $3 \times 3 \times 18 \mathrm{~mm}^{3}$. The window is glued into an extrusion in the bottom PMMA block with UV glue.

The cathode has a larger surface area $\left(52.6 \mathrm{~mm}^{2}\right)$ and is placed $20 \mathrm{~mm}$ from the membrane. The larger surface area ensures reduced ICP at the cathode compared to than at the membrane. The cathode is placed further from the membrane with a funnel shaped channel to ensure the electric field near the membrane is perpendicular and homogeneous. In each reservoir a copper wire is inserted to serve as a pseudo-reference electrode (ref 1 and ref 2 in 2.3). In the anode compartment it is placed $0.7 \mathrm{~mm}$ from the membrane and in the cathode 
compartment $7.3 \mathrm{~mm}$. By measuring the voltage difference between the sense electrodes the electrode effect (ICP and deblocking of copper oxide films [15]) can be excluded.

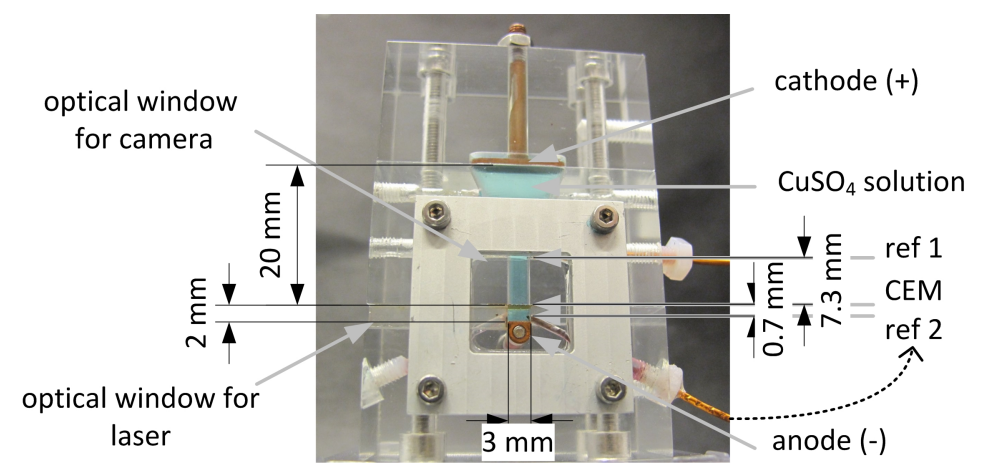

Figure 2.3: A front view of the setup. It consists of three PMMA parts screwed together with membrane and electrodes in between.

\subsubsection{Chronopotentiometry}

In chronopotentiometry $(\mathrm{CP})$, the potential difference to drive a fixed current is measured as a function of time. The current between anode and cathode is continuously measured while applying a potential difference. A feedback loop in the potentiostat adjusts the potential continuously to achieve the requested current. At the same time the voltage drop between sense and reference electrode (ref 1 and ref 2) is measured.

Between the electrodes ( $x$ direction) the electric potential drops continuously due to electrolyte resistance, but the current running through the anode is the same as through the membrane and the cathode. In other words the boundary conditions at the membrane and electrodes are known. This gives the advantage of the existence of an analytical expression for the depletion time with constant current boundary conditions, see eq. 1.29. A disadvantage of this technique is that it can take a long time before the steady state is reached.

If a current below the limiting current is applied the resistance increases slightly over time, due to arising concentration polarization. At the enriched side the resistance decreases, but at the depleted side the resistance increases. Since the resistance is inversely proportional to the concentration the total resistance increases (see Eq. 1.6). When the concentration at the membrane reaches zero the resistance of this layer goes towards infinity. The steady state (linear) gradient at which this happens is called the limiting current condition (see Eq. 1.20). Most 
measurements done in this thesis have an initial concentration of $c_{0}=10 \mathrm{mM}$ and the limiting current will be:

$$
\begin{aligned}
i_{\lim } & =\frac{z F D}{\left(\overline{t_{+}}-t_{+}\right)} \frac{c_{0}}{\delta}=2.7 \mathrm{~A} / \mathrm{m}^{2} \\
I_{\mathrm{lim}} & =i_{\mathrm{lim}} \cdot A_{\mathrm{mem}}=37 \mu \mathrm{A}
\end{aligned}
$$

In an ideal system the anion cannot leave the anode compartment because it is enclosed by two cation selective surfaces, i.e. the membrane and the anode. The limiting current condition will correspond to a linear concentration gradient with $c=0$ at the membrane and $c=2 \cdot c_{0}$ at the anode. The gradient calculated in the half cell with $\Delta x=\delta=1 \mathrm{~mm}$ and $\Delta c=c_{0}=10 \mathrm{mM}$.

The cation diffusivity $D_{C u^{2+}}=0.714 \times 10^{-9} \mathrm{~m}^{2} / \mathrm{s}$ is lower than the $D_{S O_{4}^{2-}}=$ $1.065 \times 10^{-9} \mathrm{~m}^{2} / \mathrm{s}[16]$. The average electrolyte diffusivity is taken as $D_{\mathrm{CuSO}_{4}}=$ $\left(z_{+}+\left|z_{-}\right|\right) D_{+} D_{-} /\left(z_{+} D_{+}+\left|z_{-}\right| D_{-}\right)=0.855 \times 10^{-9} \mathrm{~m}^{2} / \mathrm{s}$ [16]. The valence number $z$ of the solution is $z=2$ and the Faraday constant is $F=9.65 \times$ $10^{4} \mathrm{C} / \mathrm{mol} . \overline{t_{+}}$and $t_{+}$are the cation transport numbers for the membrane and for the bulk solution, respectively. The bulk transport number is defined as the ratio of diffusion coefficients $t_{+}=z_{+} D_{+} /\left(z_{+} D_{+}+\left|z_{-}\right| D_{-}\right)=0.4$, for $\mathrm{CuSO}_{4}$. Initially the membrane transport number is assumed ideal and taken as $\overline{t_{+}}=1$.

If a current above the limiting current is applied, the membrane interface gets depleted, eventually increasing the resistance sharply. To keep the set current,
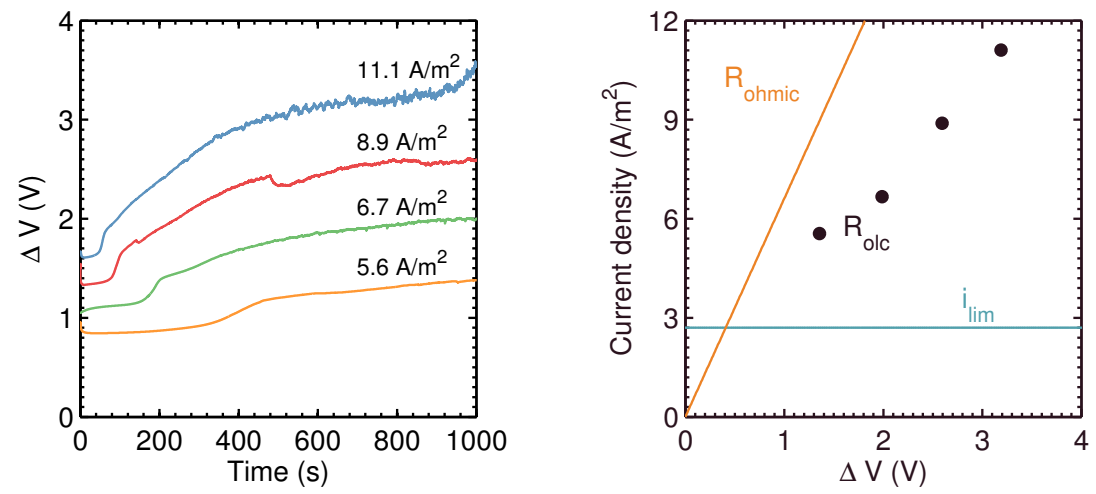

Figure 2.4: Left: Chronopotentiometric response for different current density in $10 \mathrm{mM}$ at flat CMX membrane between anode (WE) and cathode (CE). Right: Constructed IV diagram, where $i_{\text {lim }}$ is the theoretical limiting current density, $R_{\text {ohmic }}$ is the initial system resistance of a homogeneous electrolyte, and $R_{\text {olc }}$ is the resistance with a steady state OLC mixing layer (around $t \approx 800 \mathrm{~s}$ in left figure). 
the voltage is increased and overlimiting current transport mechanisms start. The voltage keeps increasing until a new steady state situation is reached.

From the chronopotentiometric response, one can extract the time, $\tau_{\mathrm{c}}$, when the membrane interface is depleted of ions and the resistance increases, as seen in figure 2.4. The theoretical transition time, $\tau_{\mathrm{s}}$, for an ion selective surface can be estimated using Sand's equation, see Eq. 1.29:

$$
\tau_{\mathrm{s}}=\frac{\pi D}{4}\left(\frac{c_{0} z F}{\overline{t_{+}}-t_{+}}\right)^{2} \frac{A^{2}}{I^{2}}
$$

The applied current $(I)$ in this thesis lies between $1-600 \mu \mathrm{A}$. The current density depends on the surface area $A$ of the ion selective surface (the membrane surface area $A_{\text {mem }}=13.5 \mathrm{~mm}^{2}$, and the cathode surface area of $A_{\text {cat }}=52.6 \mathrm{~mm}^{2}$ ).

Sand's equation is derived with the assumption that the concentration at infinite distance is $c_{0}$. In the cell the concentration in the middle of the cell is $c_{0}$. If the diffusion layer stays smaller than the half cell Sand's equation gives accurate results. Comparison with numerical calculations (solving Eq. 1.22 and Eq. 1.25) showed Sand's equation still matches if $I>1.2 \cdot I_{\text {lim }}$.

Ideally the membrane and electrode transport number is $\overline{t_{+}}=1$. Real membranes do allow a small percentage of co-ions to pass, giving $\overline{t_{+}}<1$, therefore having a higher depletion time than theoretically predicted. Shown in figure 2.5 is the comparison between the experimental and theoretical results of transitional
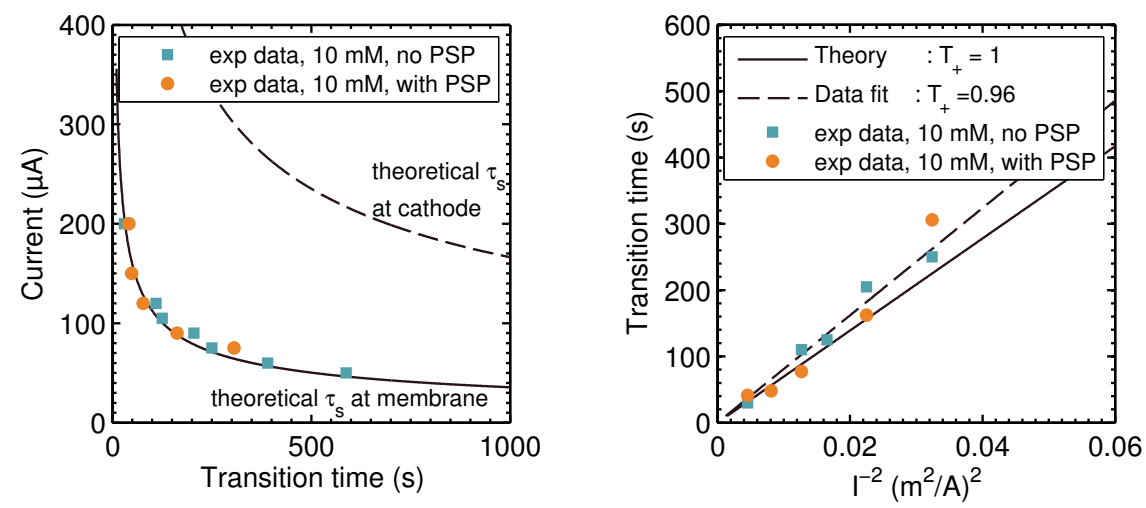

Figure 2.5: Left: Theoretical (black solid line: membrane, black dashed line: cathode) and experimental (circles: with particles, square: without particles) transition times at an CMX membrane. Right: Sand plot of theoretical (black solid line) and experimental (circles: with particles, square: without particles) transition times. The fit of the data (black dashed line) shows the average delay in transition time attributed to a non-ideal membrane transport number, $T_{+}=\overline{t_{+}}=0.96$. 
time $\tau_{\mathrm{s}}$ for the onset of limiting regime. The transition time of the cathode surface is also calculated, shown by the dashed line in figure 2.5-left. Figure 2.5-right shows the transition times for different current densities with and without seeding particles. Both results have similar transition times, with a linear relation to $J^{-2}=\left(I / A_{\text {mem }}\right)^{-2}$. The experimental transition times slightly deviate from the ones with an ideal membrane $\left(\overline{t_{+}}=1\right)$. Fitting the experimental transition times gives $\overline{t_{+}}=0.96$, which is in agreement with values reported for CMX membranes in literature [22].

\subsubsection{Chronoamperometry}

In chronoamperometry (CA), the current as a function of time at fixed potential difference is measured. The voltage between the sense and reference electrodes (ref 1 and ref 2) is continuously measured while applying a potential difference between anode and cathode. A feedback loop in the potentiostat adjusts the potential continuously to keep the voltage drop between ref 1 and ref 2 at the requested level. At the same time the current between anode and cathode is measured.

Once the potential difference is applied concentration polarization forms. When the concentration at the membrane depletes the resistance increases significantly. If the voltage is high enough, overlimiting current transport mechanisms can start and another steady state is found, see figure 2.6.
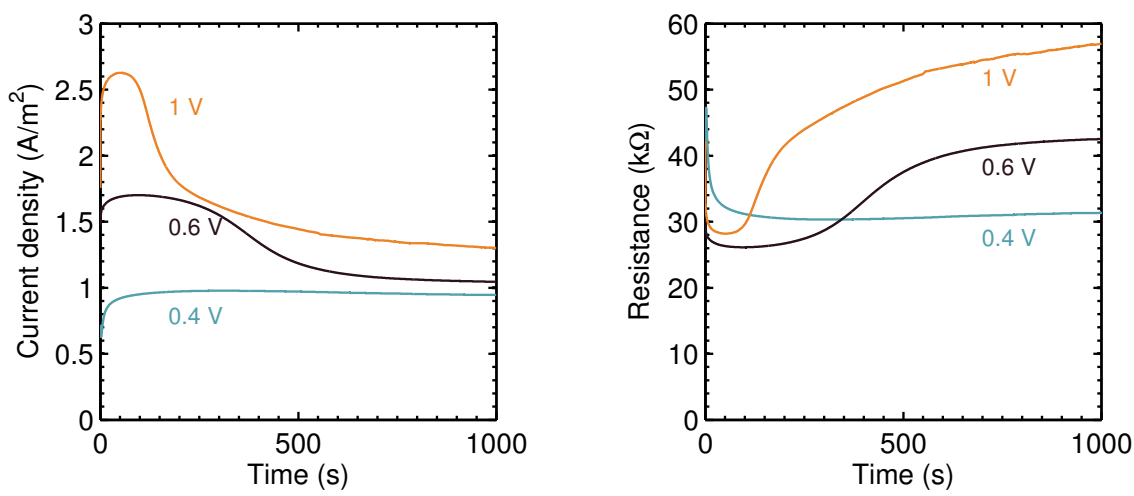

Figure 2.6: Left: Chronoamperometric response in $5 \mathrm{mM} \mathrm{CuSO}_{4}$ at $\mathrm{CMX}_{\text {membrane. }}$ Voltage difference between anode and cathode was kept fixed. Right: Resistance plot of same results, with $R(t)=\Delta V_{\text {applied }} / I(t)$.

An advantage of this technique over chronopotentiometry (CP) is that the process to equilibrium goes faster. In $\mathrm{CP}$ the voltage drop increases in time while 
the current is fixed. In CA the voltage drop is fixed while the current increases. The same current-voltage equilibrium is faster reached in CA since the energy input $(U=V I)$ is initially higher, driving the system faster to equilibrium. Another advantage is that the transient Nernst-Planck equation can be solved without assuming which species carry the current, eq. 1.21 instead of eq. 1.14. Most numerical works solve the Nernst-Planck equation with constant potential boundary conditions, for numerical simplicity (Dirichlet vs. Neumann boundary conditions) [23].

A disadvantage of the constant voltage technique is that no analytical expression for the depletion time exists. Even though depletion occurs faster than with $\mathrm{CP}$ several measurements are needed to scan the full current voltage parameter space and find the limiting current density. Another disadvantage is that the voltage drop between two electrodes also depends on the electrode processes making it difficult to distinguish between them [24].

\subsubsection{Linear voltage sweep}

During a linear voltage sweep the change in current is measured in time while the potential drop changes linearly in time. The voltage between the sense and reference electrode (ref 1 and ref 2 ) is continuously measured while applying a potential sweep between anode and cathode at a fixed scan rate, eg. $10 \mathrm{mV} / \mathrm{s}$. At the same time the current between WE en CE is measured. The step potential indicates the voltage difference between each sample, e.g. $1 \mathrm{mV}$.

In general the voltage is increased from 0 to between 2 and $5 \mathrm{~V}$. Initially the resistance is ohmic and constant. Once the concentration at the membrane becomes depleted, the resistance increases which is visible as the typical current plateau. Increasing the voltage further starts the overlimiting current transport mechanisms allowing the current to increase further [25].

The advantage of a voltage sweep is that one can quickly scan the voltage current parameter space $[26,27]$. A disadvantage is that the outcome of this method depends on the scan rate. At faster scan rates the plateau occurs at a higher current, see figure 2.7. The interface concentration gets depleted faster, so diffusion has less time to expand the concentration gradient. For the IV curve to represent the steady state, the voltage steps should be sufficiently slow for the gradient to become linear via diffusion.

\subsection{Flow measurements}

This section describes the experimental techniques to quantify the fluid velocity in the membrane boundary layer. Knowing the velocity vector field allows one 

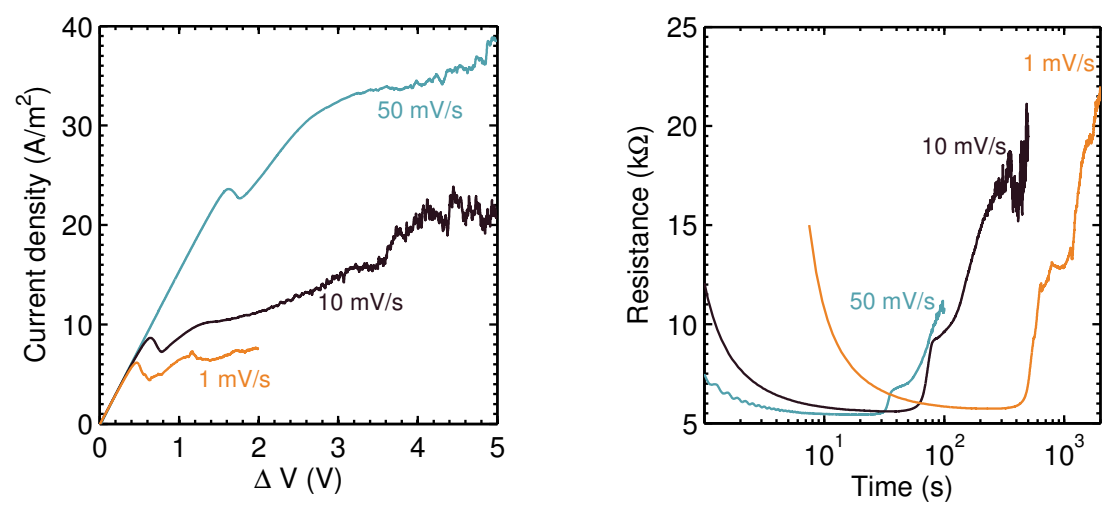

Figure 2.7: Left: Linear voltage sweep at difference scanrates in $10 \mathrm{mM} \mathrm{CuSO}_{4}$ at a CMX membrane. Right: Resistance vs time plots of the same results, with time on a logarithmic scale. Initial resistance drop is associated to electrode effects. Depletion occurs slower at lower scan rate.

to distinguish how and when electroconvection and/or gravitational convection occurs. The fluid velocity is an essential component in the advective mass transfer term in the Nernst-Planck equation (eq. 1.8). Once also the concentration inside the fluid is known, one can estimate the advective ion transport. The electroconvective mixing in the boundary layer without pressure driven flow can have typical velocity of $\mathcal{O}\left(10^{-5} \mathrm{~m} / \mathrm{s}\right)$ in the thin layer of $\mathcal{O}\left(10^{-3} \mathrm{~m}\right)$.

Flow measurements can be separated into three groups:(a) kinematic, (b) dynamic, and (c) physical. The kinematic techniques (a) follow the movement of a marked section of the fluid. This can be particles, bubbles or dyes. When light or sound is used this typically does not disturb the system much, but the immersed particle might [28]. Dynamic measurements (b) record the interaction between the fluid and a measuring probe. The probe can for example be mechanical like a rotor that spins due to the pressure or electromagnetic like the change in a pulsed magnetic field due to the flow [29]. For physical methods (c) the velocity is derived indirectly from physical properties in the liquid. An example is a hot wire probe from which the heat transfer is measured which depends on the convection, thus fluid velocity [29].

Optical methods have always been used throughout history. By following leaves in the water one can estimate the flow velocity. Recording the light reflection from objects emerged in a liquid is the basis of optical kinematic techniques. By adding mica particles to water and flowing the water in a controlled fashion along different shapes allowed Prandtl to investigate fluid properties [28]. With the improvement of optics, light sources (lasers), electronics, video and com- 
puter techniques it became possible to observe in more and more detail the fluid phenomena and quantify the velocities.

The investigation of the microfluidic properties of the boundary layer are difficult due to the scale and its location next to the membrane. The first optical recordings of electroconvection at a membrane were measurements with suspended latex particles $(0.2 \mu \mathrm{m})[30,31]$. A laser beam was send through the boundary layer in a transparent cell onto a photodetector. The detected fluctuation of the scatted light increased in the overlimiting current regime. This indicated motion in the boundary layer proportional to the degree of OLC. With a more sophisticated light scattering technique the flow in the mixing layer was estimated to be $\mathcal{O}\left(10^{-6} \mathrm{~m} / \mathrm{s}\right)[32]$.

Direct observations of microscale flow became possible with the advances in microscopy. The availability of these observations led to the discovery of RayleighBénard convection in an electrochemical cell [33]. This discovery was qualitative, just the observation by eye through the microscope. Only in 2008 the first movie of particle seeded electroconvection near an ion exchange membrane was made and it showed no chaotic mixing [15]. Related phenomena were also investigated in micro-nano channels [34, 35]. Velocity estimates could be made, but no qualitative velocity field could be extracted and the flow dynamics within the vortices remained hidden. This thesis shows quantitative flow fields by employing a particle image velocimetry technique on a microscale. By extending the image acquisition frame rate together with appropriate seeding density it was possible to extract the velocity flow field in more detail then done before.

\subsubsection{Particle Image Velocimetry (PIV)}

When two images of particles are obtained with a known time difference and scale it is possible to extract the velocity. In digital particle image velocimetry (DPIV) such images are taken and analysed with special developed computer algorithms. By careful synchronization of illumination and recording these images can be captured. In convectional PIV the illumination is done with a laser sheet to illuminate only a cross-section of a volume, as shown in figure 2.2. The line of sight of a camera with proper lenses is placed perpendicular to the light sheet and focused within to capture only motion of the two dimensions of the plane. This ensures the illuminated particles are well focused and the background noise from out of focus particles is minimized. For more details on recording techniques the reader is referred to $[28,36]$ and chapter 3,4 and 5 of this thesis.

Once two images are captured they are analysed via a cross-correlation method. The image are divided into several interrogation areas. Inside these areas the average displacement of the group of particles is used to calculate the local velocity, 
see figure 2.8. Every pixel has a intensity value such that the particles have an higher value than the background, typically white particles on a black background The interrogation window is actually a matrix with these values. The window of one of the two pictures is multiplied with the other. The sum of the product of the overlapping pixels is calculated. The shift with the highest values represents the most similar pattern. For this technique to be successful the particle density has to be high enough to have everywhere sufficient particles to extract the velocity $[37,38]$. When the particle density is low the displacement of the individual particles can be traced by particle tracking velocity (PTV) algorithms [38, 39]. When the density becomes too high the particles become indistinguishable and form speckles, which can also be used to extract information [40].

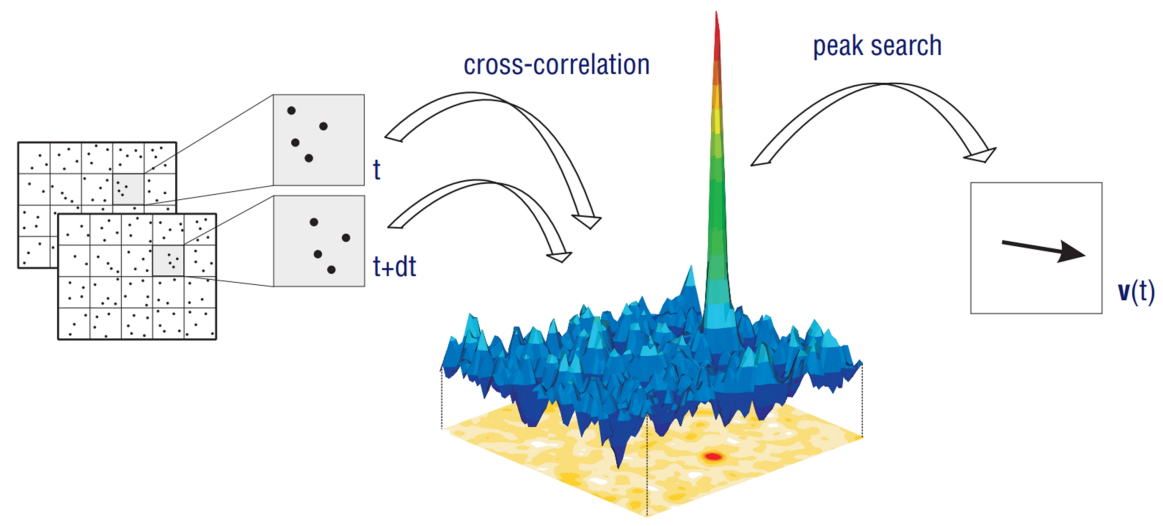

Figure 2.8: Two images are divided in multiple correlation windows. For each window the mean displacement of the particles is taken as the highest peak in the correlation plane. After the complete velocity vector map is determined erroneous vectors are removed using a nearest neighbour algorithm. Image courtesy of LaVision.

When microscale flow is imaged one speaks of $\mu \mathrm{PIV}$. Due to the small scale, difficulties arise. To image the flow with micrometer resolution small particles compared to the wavelength of light are needed and Mie light reflection is not adequate. To solve this fluorescent particles are often used [41]. An additional benefit of fluorescence is the ability to filter out the reflection of objects other than the particles, for example the side walls of a microchannel. With decreasing particle size random Brownian motion of the particle has to be taken into account. It is common to use temporal and/or spacial averaging to filter out the random particle motion [41]. In microfluidic systems it is often difficult or impossible to create a laser sheet with micrometer thickness. Therefore the flow is volume illuminated and the depth of focus of the microscopic objective is used to take 
a planar image [41]. Details on these developments can be found in the several review articles [42-45].

\subsubsection{Flow velocity errors induced by tracer particles}

Particle tracking is often described as a non-invasive technique [28]. This means that the presence of the particle does not influence the flow properties significantly, for example conductivity and viscosity. At low seeding density this is often the case, but the system should be measured with and without particles to see if this is actually the case. A difference in density between particle and fluid gives rise to inertia effects. This can be estimated with the Stokes drag equation [28]:

$$
U_{\mathrm{l}}=U_{\mathrm{p}}-U_{\mathrm{f}}=d_{\mathrm{p}}^{2} \frac{\left(\rho_{\mathrm{p}}-\rho_{\mathrm{f}}\right)}{18 \mu} \mathbf{a}
$$

were $U_{\mathrm{l}}, U_{\mathrm{p}}$ and $U_{\mathrm{f}}$ are the lag, particle and fluid velocity, $d_{\mathrm{d}}$ is the particle diameter, $\rho_{\mathrm{p}}$ and $\rho_{\mathrm{f}}$ are the density of the particle and fluid, respectively, and $\mu$ is the dynamic viscosity. The same equation can be used to calculate the velocity lag when the fluid is accelerating with $\mathbf{a}\left[\mathrm{m} / \mathrm{s}^{2}\right]$, or to calculate the gravitational settling speed due to the gravitation force with acceleration g. Particles with similar density to the fluid are preferred since they display less lag and less gravitational settling. Also reducing the particle size reduces the effect of these body forces.

With reducing particle size surface related forces have a larger influence on particle motion. A wide range of particle motion phenomena have been observed, caused by gradients in temperature, electric potential, pressure and concentration [29, 46-48]. These phenomena can give a discrepancy between the particle motion and fluid velocity. The most important in this work are motion driven by the electric field, (di)electrophoresis. The electrophoretic drift velocity of a nonconducting particle is described by the Helmholtz-Smoluchowki equation [29]:

$$
U_{\mathrm{e}}=\frac{\varepsilon \zeta E_{\|}}{\mu}
$$

were $U_{\mathrm{e}}$ is the electrophoretic velocity, $\varepsilon$ is the electric permittivity of the liquid, $\zeta$ is the zeta potential of the particle, $E_{\|}$is the electric field applied along the surface charge and $\mu$ is the dynamic viscosity of the liquid.

When a charged particle is immersed in an electrolyte the charged surface attracts ions of opposite charge, which form an electric double layer and neutralize the surface charge, which is called electric shielding or screening. Due to thermal fluctuations the shielding layer extends outwards. The distance from which the 
ions can flow ('slip') along the particle is defined as the shear plane. The potential difference between this shear plane and the electrolyte solution is called the zeta potential $(\zeta)$. Particles with low zeta potential are close to neutral and undergo a lower electrophoretic motion. The zeta potential does depend on the ion concentration of solution. At low electrolyte concentrations less ions are present to screen the fixed charge of the particle. Therefore, the particle has more effective charge and is more susceptible for electrophoresis.

Next to the particle inertia, the size also effects the particle visibility. The reflection of light from a particle is described by Mie scattering theory [28]. The reflected light intensity is proportional to $d_{p}^{2}$. The intensity also depends on the angle of the particle towards the light source and the particle shape [28, 49]. To minimize the intensity fluctuation due to internal rotation spherical particles are preferred and particle aggregates should be avoided. In summary, the light scattering and body forces decrease with decreasing particle diameter, while the surface effects become more present. In PIV no ideal particle exists, so a suitable size has to be determined depending on the experimental conditions and the mismatch between flow and particle motion has to be estimated.

\subsubsection{Flow velocity errors induced by vector field analysis}

This section provides a description of the systematic errors in the PIV vector field. These can be categorized into two groups: (a) mismatch between particle displacement and fluid velocity, and (b) falsely correlated interrogation area displacement [28]. As discussed in the previous section the particles can undergo additional transport and therefore do not represent the fluid velocity accurately. In this thesis particle lag, due to the gravitational field, and electrophoretic motion are the dominant effects.

A side-effect on particle lag and forces is that the seeding density becomes heterogeneous. While initially the particles are aimed to be homogeneously dispersed, these forces can create particle voids. This hinders the extraction of information out of these regions $[50,51]$. A common example is the void formation due to the gravitational settling or rising of particles. Also in vortices when the streamlines bent strongly and velocity gradients are present a small deviation can give rise to particle void volumes, see figure 2.9. When an area is void of particles the cross-correlation does not give a strong peak. The image shift in all directions gives a similar result, in contrast what is shown in figure 2.8.

The particle density can also be too high at a certain region for a clear correlation peak to be observed. When strong gradients are present in the interrogation window no linear displacement can capture the motion. This happens for example in vortex centres and the boundary between the solid and liquid (flow) region 

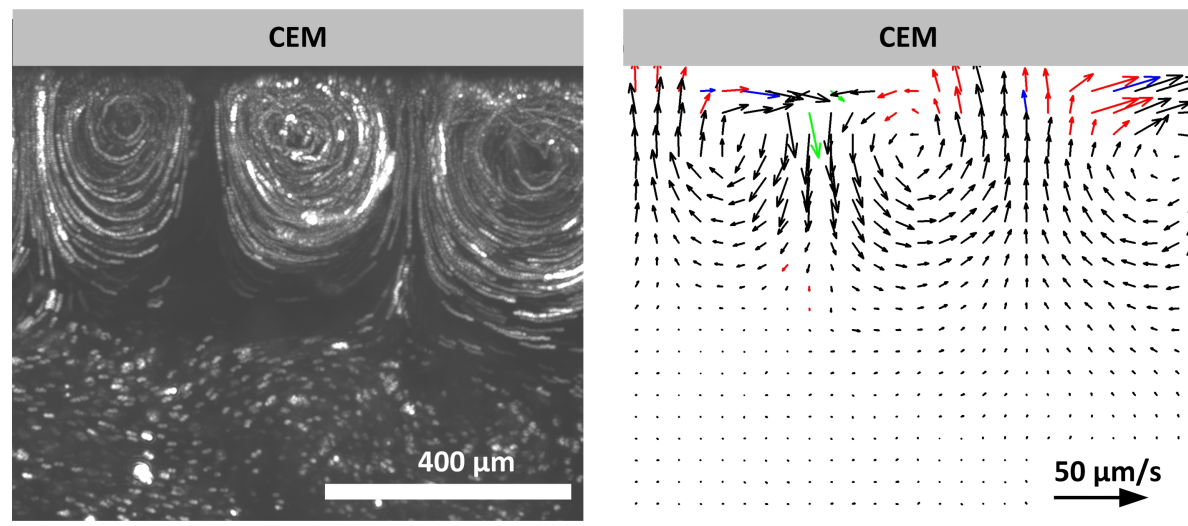

Figure 2.9: Left: an overlay of 100 images showing the particle pathlines over 5s. Areas void of particles are present. Right: a vector velocity field derived from two images. The black arrows are value that passed the post-processing test. The green arrows are derived from the second highest correlation peak. The red arrows are interpolated values from the nearest neigbors. Image shows electroconvective vortices at a CMX membrane with $i=8.9 \mathrm{~A} / \mathrm{m}^{2}=3.3 \cdot i_{\text {lim }}, \mathrm{t}=300 \mathrm{~s}$.

[52]. Another reason why boundaries are hard to quantify is that only part of the interrogation window is filled with moving particles and contains high velocity gradients. This gives rise to false linear correlations inside the interrogation window. Light reflection from the solid interface can be bright and dominant in the correlation calculation.

To overcome the false correlations at the interface the regions without flow are commonly excluded from the correlation calculation, by masking. The area does not induce false vectors and these areas are also excluded from calculations of the average. Another way to increase the particle signal to background noise ration is by subtracting the background to enhance the particle contrast. There is a wide range of image preprocessing algorithms designed to enhance the contrast [53]. Improving the signal to noise ratio may lead to loss of information. By enhancing the particle contrast blurry and out of focus particles can be either neglected or wrongly considered. The displacement of these particles might not represent the movement within the cross-section targeted by the laser sheet or optical focus. But even if image preprocessing is done optimally regions without particle keep existing and flow shapes smaller then the interrogation window cannot be resolved and erroneous velocity vectors will emerge.

To check for outliers (erroneous vectors) the most common test is to compare the vector with the median value of its nearest neighbours [54]. This value 
is less sensitive to neighbouring outliers compared to a normal average. More sophisticated techniques look also at the quality of the highest correlation peak [28]. Once a vector does not pass such a test an alternative vector can be found, either by taking another correlation peak or by interpolation from the nearest neighbours, see figure 2.9-right. Notice that the validity of the boundary vectors is more difficult to check because they have less direct neighbours. Often the boundary vectors are extrapolated from the values of the vectors in the central regions [52].

Another way to reduce the influence of erroneous vectors is by using the average of several vector fields. In steady flows this is frequently employed [28]. In microfluidics it also helps to smooth out the Brownian motion of the very small seeding particles, $\mathcal{O}\left(10^{-7} \mathrm{~m}\right)[41,45]$. Large erroneous vectors still can have a big influence. Averaging the correlation peak can give a more accurate vector, although this is a computational costly task [55].

The image correlation in conventional PIV gives a $2 \mathrm{D}$ velocity field, while the real velocity field is always $3 \mathrm{D}$. Also the setup used in this thesis is a planar PIV setup equipped with one camera perpendicular to a laser sheet, see figure 2.2. It traces the in-plane motion $\left(v_{x}\right.$ and $v_{y}$ ), while particle motion can a significant $v_{z}$ component. This fact is observed if the particle intensity changes in time, indicating a particle movement perpendicular to the laser sheet. Different systems have been proposed to measure the third velocity component. In the case of stable flows one can make images at different depth [28]. By using two cameras it is possible the extract the third velocity component in the light sheet, stereo PIV [28]. With four cameras the motion in a illuminated volume can be determined, tomographic PIV $[56,57]$. Recent advances are made with a single camera equipped with an cylindrical objective. The particles shape becomes ellipsoidal depending on their position along the $z$ axis with respect to the focal plane, which can be used to determine $v_{z}[44,56]$.

\subsection{Concentration measurements}

The previous sections described how to extract electric resistance and fluid flow information. This section completes the picture with concentration measurements such that all terms in the Nernst-Planck equation (eq. 1.21) can be experimentally investigated. Concentration and velocity field allow to quantify the advective and diffusive transport. The migration term can be calculated from the local electric potential gradient and concentration, or back calculated from the current.

In electrodialysis it is common to measure the concentration before and after 
the separation [1]. Conductivity probes relate the electrical resistance of the electrolyte to the concentration, see eq. 1.6. To determine the ion composition in more detail, samples can be taken and used for destructive measurements, like ion coupled plasma or ion chromatography [58]. To determine the concentration next to the membrane, conductivity measurements with microprobes were used by Choi et al. [59]. They varied the micro-electrode position with steps of $10 \mu \mathrm{m}$ and the change in resistance was mapped to the local concentration. They showed the concentration boundary layer was approximately $300 \mu \mathrm{m}$ thick. When overlimiting current was applied the resistance fluctuated but also showed a higher interface concentration than in the limiting case, indicating some mixing of the boundary layer.

To get a full field view of the concentration profile in a membrane channel Shaposhnik et al. used a laser-interferometric method [60, 61]. A laser beam was send through the electrolyte channel and the difference in optical path as a function of location is measured. The optical path depends on the average density along the path. This method can quantify gradients, but not absolute concentration.

Another way to determine the concentration is by looking at the light absorption and reflection of dye molecules that are dependent on the ion concentration. In spectrometry the absorption of a specific wavelength is measured and compared to a calibration curve [62]. Special dyes react with the compound of interest and change color depending on the compound's concentration. To visualize ion concentration gradients charged dyes can be added, e.g. Alexa Fluor ${ }^{\circledR}$ 488. The dye is initially homogeneously dispersed in the electrolyte. When the electric field is turned on electromigration starts and at the charge selective surfaces a dye concentration gradient forms. The dye concentration profile can be representative for the electrolyte concentration profile. By adding a strong light absorbing colored charged dye the ion depletion near copper electrodes was visualized [63].

Nowadays it is preferred to use charged fluorescent dyes, since this gives allows to filter out the background light $[64,65]$. The fluorescent molecules absorb photons with a specific wavelength. The energy of the incoming photon excites an electron of the molecule to a higher orbital where is stays for a typical lifetime of 1 $10 \mathrm{~ns}$. When the electron returns to the ground-state orbital a photon is emitted, although with a lower energy (higher wavelength). Some of the incoming energy is used to effect the structure of the molecule or is lost due to interaction with the environment [66]. With the use of special optical crystals the fluorescent light can be distinguished from the incoming light. In figure 2.10 a typical fluorescent setup is shown in which blue light $(\sim 490 \mathrm{~nm})$ is used to excite a dye (e.g. Alexa Fluor $\left.{ }^{\circledR} 488\right)$ and the emitted green light $(\sim 510 \mathrm{~nm})$ is recorded on a camera.

In ICP measurements the absence of emitted light in the boundary layer is 




Figure 2.10: Left: Jablonski diagram indication the fluorescent process [66]. Right: schematic setup to detect the fluorescent light coming from the sample using a filter set.

frequently associated to the absence of ions $[34,64,65]$. It has to be noticed the diffusion coefficient of dye is typically different than that of the ions, therefore the dye concentration profile cannot be linked one to one with the ion concentration profile [67]. However, the fluorescent light intensity depends on more factors. A well known effect is the influence of the local $\mathrm{pH}$ to the fluorescent efficiency ,also called quantum yield, of the dye molecule [68]. The decrease of efficiency, also known as quenching, can occur due to the presence of molecules like protons, oxygen and proteins [66]. In time, the efficiency of the dye can also decrease by photobleaching; the light can alter the molecule permanently, thereby decreasing the amount of active molecules [68]. Another reason why it is hard to quantify the light intensity to ion concentration is due to a varying light absorption at different points in the optical plane. The optical access to the electrolyte can be inhomogeneous due to system geometry. An example is a cylindrical channel where the optical path to the middle of the channel has a different fluid/solid ratio compared to the edge. The light absorption in the electrolyte can also dependent on the ion concentration. A good example is the blue color of $\mathrm{CuSO}_{4}$ as seen in figure 2.1. In case of concentration polarization the higher ion concentration increases light absorption, while a higher dye concentration increases the fluorescence. These effects prevented the quantification of ICP inside a $\mathrm{CuSO}_{4}$ solution with a fluorescent light intensity method and allowed for only qualitative information as to the nature of the depletion zone. 


\subsubsection{Fluorescence Lifetime Imaging Microscopy (FLIM)}

To overcome the limitations of the light intensity analysis, as discussed in the previous section, a fluorescent lifetime methodology was chosen. Fluorescent emission of a molecule is a stochastic process [66]. The probability of the emission follows a decaying exponential form. The lifetime is the average delay between absorption and emission and depends on interaction with the local surrounding. If the ion concentration varies, the lifetime can be calibrated to the concentration. By measuring the lifetime, effects like photostability and medium transparency can be excluded. The lifetime also does not depend on the dye concentration. To measure the lifetime only a minimum quantity of light is needed to produce a signal [66]. These properties have made fluorescence lifetime imaging microscopy (FLIM) a popular tool to determine local ion concentration (e.g. $\mathrm{Ca}^{2+}, \mathrm{Cu}^{2+}$, $\left.\mathrm{Cl}^{-}, \mathrm{pH}\right)$ in cellular processes [66, 69-71]. In microfluidics it has also been used to study ion transport in confined systems (e.g. oxygen transport in water or diffusion in nanopores) $[72,73]$.
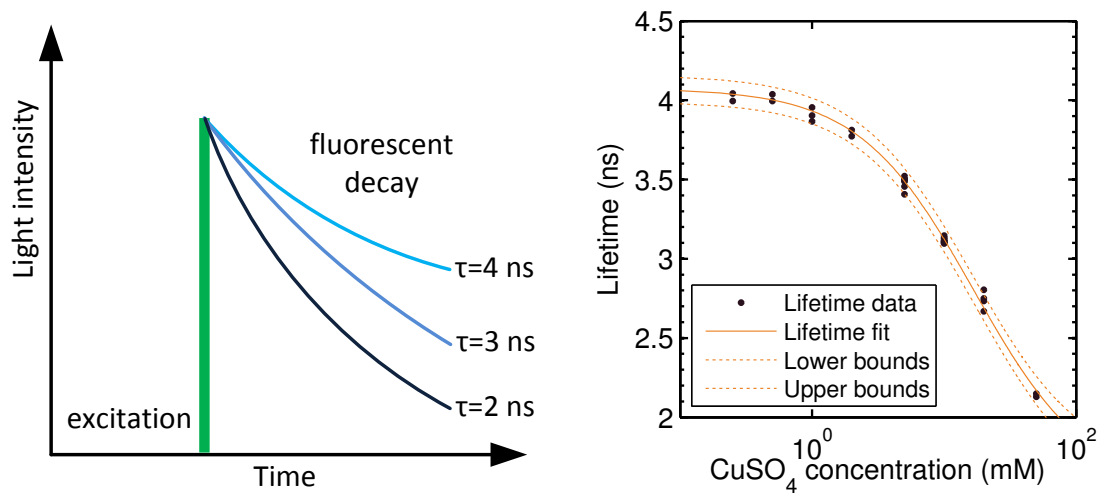

Figure 2.11: Left: After a sample gets excited with with a pulse light source the fluorescent decay rate depends on the lifetime (adopted from [66]). Right: Lifetime vs concentration calibration curve on semi-log scale (own work).

The fluorescence lifetime can be measured by modulating the intensity of the exciting light source. The intensity follows a sinusoidal wave with a period in the order of the lifetime. This will give the emitted fluorescent light also a periodic intensity, but with a phase shift and a different modulation depth due to the lifetime of the fluorescent molecules. The phase shift is chosen such that small changes in the lifetime of the sample result in large changes in emitted phase. 
The optimal frequency can be calculated via:

$$
f=\frac{1}{2 \pi \tau}
$$

where $f$ is the modulation frequency and $\tau$ is the fluorescent lifetime. A typical lifetime of $4 \mathrm{~ns}$ should be illuminated with a $40 \mathrm{MHz}$ frequency [74]. The fluorescent light is directed to an intensified CCD camera with a signal amplifier. The signal amplifier is modulated in the same frequency as the light source. The voltage is modulated around a cutoff voltage such that the amplification is periodically on and off. This way the camera can collect photons with the same delay over multiple periods till it has enough counts to have a good signal to noise ratio.

By making multiple images with a different phase difference between the light source modulation and the camera modulation the different in phase and modulation depth of every pixel can be fitted. This shift is compared to a reference sample with a known shift, from this the average lifetime of the volume represented by the pixel is calculated. From a lifetime versus concentration calibration curve each pixel value can be converted to concentration, see figure 2.11-right for an example calibration curve. The obvious benefit of this technique is that the lifetime is independent on intensity. A drawback is that multiple images are needed to fit the lifetime. This limits the temporal resolution of the system. To make one image only a part of the modulated light is used. To have enough photon counts the dye concentration can be increased or the recording time can be increased. More details on this technique can be found in $[66,69,72,75]$.

\subsection{Experimental techniques used in each chapter}

In Chapter 3 two point chronopotentiometric measurements using only the anode and cathode are carried out. The anode serves as a working electrode (WE) and a sense electrode (SE). The cathode serves as the counter electrode (CE) and the reference electrode (RE). Simultaneously to the electrical measurements, the fluid velocity is extracted via particle image velocimetry (PIV).

In Chapter 4 the setup is extended to a three electrode system with a reference electrode in the cathode reservoir to exclude the resistance increase due to ICP at the cathode. Again chronopotentiometric measurements are performed with the anode as a WE and SE. The cathode serves as a the CE while the copper wire acts as RE. Again simultaneous PIV measurements are conducted. In separate experiments the resistance change was recorded while the concentration profile was imaged using FLIM with the Alexa Fluor ${ }^{\circledR} 488$ dye. The dye has a measurable change in lifetime in the studied range of $\mathrm{CuSO}_{4}$ concentration. 
In Chapter 5 the setup is extended to a four electrode system with also a $\mathrm{SE}$ in the anode reservoir to exclude both the anode and cathode resistance. Here chronoamperometric measurements are presented. The anode is the WE, the cathode is the $\mathrm{CE}$, the anode compartment copper wire is the SE and the cathode compartment with copper wire is the RE. The flow is imaged with PIV or the concentration with FLIM. 



\section{Bibliography}

[1] H. Strathmann, Ion exchange membrane separation processes - membrane science and technology series 9, 1st editio ed. (Elsevier, 2004), No. 9. p.43, 59 .

[2] C. W. Tobias, M. Eisenberg, and C. R. Wilke, Diffusion and convection in electrodialysis - A theoretical review, Journal of the Electrochemical Society 99, 359 (1952). - p.43.

[3] N. W. Rosenberg and C. E. Tirrell, Limiting Currents in Membrane Cells, Industrial \& Engineering Chemistry 49, 780 (1957). - p..

[4] A. A. Sonin and M. S. Isaacson, Optimization of flow design in forced flow electrochemical systems, with special application to electrodialysis, Industrial and Engineering Chemistry: Process and Design Development 13, 241 (1974). - p.43.

[5] V. V. Nikonenko, N. D. Pismenskaya, E. I. Belova, P. Sistat, P. Huguet, G. Pourcelly, and C. Larchet, Intensive current transfer in membrane systems: Modelling, mechanisms and application in electrodialysis, Advances in Colloid and Interface Science 160, 101 (2010). — p.44.

[6] J. Veerman, M. Saakes, S. J. Metz, and G. J. Harmsen, Reverse electrodialysis: Evaluation of suitable electrode systems, Journal of Applied Electrochemistry 40, 1461 (2010). - p.44.

[7] B. Gavish and S. Lifson, Membrane polarisation at high current densities, Journal of the Chemical Society, Faraday .. 75, (1979). — p.44.

[8] I. Rubinstein and L. Shtilman, Voltage against current curves of cation exchange membranes, Journal of the Chemical Society, Faraday Transactions 2 75, 231 (1979). - p..

[9] I. Rubinstein, A. Warshawsky, L. Schechtman, and O. Kedem, Elimination of acid-base generation ('water-splitting') in electrodialysis, Desalination 51, 55 (1984). - p.44.

[10] K. S. Spiegler, Polarization at ion exchange membrane-solution interfaces, Desalination 9, 367 (1971). — p.44.

[11] J. J. Krol, M. Wessling, and H. Strathmann, Concentration polarization with monopolar ion exchange membranes: current-voltage curves and water dissociation, Journal of Membrane Science 162, 145 (1999). — p.44. 
[12] I. Rubinstein, E. Staude, and O. Kedem, Role of the membrane surface in concentration polarization at ion-exchange membrane, Desalination 69, 101 (1988). - p.44.

[13] F. Maletzkl, H. Rdsler, E. Staude, F. Maletzki, H.-W. Rösler, and E. Staude, Ion transfer across electrodialysis membranes in the overlimiting current range: stationary voltage current characteristics and current noise power spectra under different conditions of free convection, Journal of Membrane Science 71, 105 (1992). - p..

[14] H. Rösler, F. Maletzki, and E. Staude, Ion transfer across electrodialysis membranes in the overlimiting current range: chronopotentiometric studies, Journal of membrane science $\mathbf{7 2}, 171$ (1992). - p..

[15] S. Rubinstein, G. Manukyan, A. Staicu, I. Rubinstein, B. Zaltzman, R. Lammertink, F. Mugele, and M. Wessling, Direct Observation of a Nonequilibrium Electro-Osmotic Instability, Physical Review Letters 101, (2008). - p.44, 47, 53 .

[16] D. E. Lide, CRC Handbook of Chemistry and Physics, 76th ed. (CRC Press, 1996), pp. 5-90. - p.44, 45, 48.

[17] M. Rosso, Electrodeposition from a binary electrolyte: new developments and applications, Electrochimica Acta 53, 250 (2007). — p.44.

[18] J. West, Electrodeposition and corrosion processes - John Michael West Google Boeken, 2nd ed. (Van nostrand reinhold company ltd, 1970), p. 206. - p..

[19] J. W. Dini, Electrodeposition : the materials science of coatings and substrates (Noyes Publications, 1993), p. 367. - p.44.

[20] H. Strathmann, Electrodialysis, a mature technology with a multitude of new applications, Desalination 264, 268 (2010). - p.45.

[21] C. Larchet, G. Eigenberger, A. Tskhay, K. Tastanov, and V. Nikonenko, Application of electromembrane technology for providing drinking water for the population of the Aral region, Desalination 149, 383 (2002). — p.45.

[22] P. Długołęcki, B. Anet, S. J. Metz, K. Nijmeijer, and M. Wessling, Transport limitations in ion exchange membranes at low salt concentrations, Journal of Membrane Science 346, 163 (2010). - p.50.

[23] C. L. Druzgalski, M. B. Andersen, and a. Mani, Direct numerical simulation of electroconvective instability and hydrodynamic chaos near an ion-selective surface, Physics of Fluids 25, 110804 (2013). - p.51.

[24] V. Fleury, J.-N. Chazalviel, and M. Rosso, Theory and experimental evidence of electroconvection around electrochemical deposits, Phys. Rev. Lett. 68, 2492 (1992). - p.51.

[25] V. V. Nikonenko, A. V. Kovalenko, M. K. Urtenov, N. D. Pismenskaya, J. Han, P. Sistat, and G. Pourcelly, Desalination at overlimiting currents: 
State-of-the-art and perspectives, Desalination 342, 85 (2014). — p.51.

[26] G. Yossifon and H.-C. Chang, Changing nanoslot ion flux with a dynamic nanocolloid ion-selective filter: Secondary overlimiting currents due to nanocolloid-nanoslot interaction, Physical Review E 81, (2010). — p.51.

[27] D. Deng, E. V. Dydek, J.-H. Han, S. Schlumpberger, A. Mani, B. Zaltzman, and M. Z. Bazant, Overlimiting current and shock electrodialysis in porous media., Langmuir : the ACS journal of surfaces and colloids 29, 16167 (2013). - p.51.

[28] M. Raffel, C. Willert, and J. Kompenhans, Particle image velocimetry: a practical guide, 2nd ed. (Springer, 2007), p. 448. - p.52, 53, 55, 56, 58.

[29] R. F. Probstein, Physicochemical hydrodynamics : an introduction (Wiley, 1994), p. 400 . - p.52, 55.

[30] S. Reich, B. Gavish, and S. Lifson, Visualization of hydrodynamic phenomena in the vicinity of a semipermeable membrane, Desalination 24, 295 (1977). - p.53.

[31] S. Lifson, B. Gavish, and S. Reich, Flicker noise of ion-selective membranes and turbulent convection in the depleted layer, Biophysics of structure and mechanism 4, 53 (1978). — p.53.

[32] Q. Li, Y. Fang, and M. Green, Turbulent light scattering fluctuation spectra near a cation electrodialysis membrane, Journal of Colloid and Interface Science 91, 412 (1983). - p.53.

[33] W. J. Ward and O. H. L. Blanc, Rayleigh-Benard Convection in an Electrochemical Redox Cell, No. 4669 in New Series, Science 225, 1471 (1984). - p.53.

[34] S. Kim, Y.-C. Wang, J. Lee, H. Jang, and J. Han, Concentration Polarization and Nonlinear Electrokinetic Flow near a Nanofluidic Channel, Physical Review Letters 99, 044501 (2007). — p.53, 60.

[35] R. Kwak, G. Guan, W. K. Peng, and J. Han, Microscale electrodialysis: Concentration profiling and vortex visualization, Desalination 308, 138 (2013). - p.53.

[36] P. Willems, N. G. Deen, A. J. B. Kemperman, R. G. H. Lammertink, M. Wessling, M. van Sint Annaland, J. A. M. Kuipers, and W. G. J. van der Meer, Use of Particle Imaging Velocimetry to measure liquid velocity profiles in liquid and liquid/gas flows through spacer filled channels, Journal of Membrane Science 362, 143 (2010). — p.53.

[37] J. Westerweel, Fundamentals of digital particle image velocimetry, Measurement Science and Technology 8, 1379 (1997). — p.54.

[38] C. J. Kähler, S. Scharnowski, and C. Cierpka, On the resolution limit of digital particle image velocimetry, Experiments in Fluids 52, 1629 (2012). - p.54. 
[39] C. Cierpka, M. Rossi, R. Segura, and C. J. Kähler, On the calibration of astigmatism particle tracking velocimetry for microflows, Measurement Science and Technology 22, 015401 (2010). - p.54.

[40] C. E. Willert and M. Gharib, Digital particle image velocimetry, Experiments in Fluids 10, 181 (1991). - p.54.

[41] J. G. Santiago, S. T. Wereley, C. D. Meinhart, D. J. Beebe, and R. J. Adrian, A particle image velocimetry system for microfluidics, Experiments in Fluids 25, 316 (1998). - p.54, 55, 58.

[42] R. Lindken, M. Rossi, S. Große, and J. Westerweel, Micro-Particle Image Velocimetry (PIV): Recent developments, applications, and guidelines, Lab on a Chip 9, 2551 (2009). — p.55.

[43] S. T. Wereley and C. D. Meinhart, Recent Advances in Micro-Particle Image Velocimetry, Annual Review of Fluid Mechanics 42, 557 (2010). — p..

[44] C. Cierpka and C. J. Kähler, Particle imaging techniques for volumetric three-component (3D3C) velocity measurements in microfluidics, Journal of Visualization 15, 1 (2012). - p.58.

[45] D. Sinton, Microscale flow visualization, Microfluidics and Nanofluidics 1, 2 (2004). - p.55, 58 .

[46] H. Bruus, Theoretical microfluidics (Oxford University Press, 2008). p.55.

[47] T. M. Squires and S. R. Quake, Microfluidics: Fluid physics at the nanoliter scale, Reviews of Modern Physics 77, 977 (2005). - p..

[48] M. Z. Bazant, M. S. Kilic, B. D. Storey, and A. Ajdari, Towards an understanding of induced-charge electrokinetics at large applied voltages in concentrated solutions., Advances in colloid and interface science 152, 48 (2009). - p.55.

[49] a. Melling, Tracer particles and seeding for particle image velocimetry, Measurement Science and Technology 8, 1406 (1997). — p.56.

[50] S.-J. Liu, H.-H. Wei, S.-H. Hwang, and H.-C. Chang, Dynamic particle trapping, release, and sorting by microvortices on a substrate, Physical Review E 82, 026308 (2010). - p.56.

[51] Y. Green and G. Yossifon, Dynamical trapping of colloids at the stagnation points of electro-osmotic vortices of the second kind, Physical Review E 87, 033005 (2013). - p.56.

[52] C. J. Kähler, S. Scharnowski, and C. Cierpka, On the uncertainty of digital PIV and PTV near walls, Experiments in Fluids 52, 1641 (2012). — p.57, 58.

[53] M. Rossi, R. Segura, C. Cierpka, and C. J. Kähler, On the effect of particle image intensity and image preprocessing on the depth of correlation in microPIV, Experiments in Fluids 52, 1063 (2012). - p.57. 
[54] J. Westerweel and F. Scarano, Universal outlier detection for PIV data, Experiments in Fluids 39, 1096 (2005). - p.57.

[55] C. D. Meinhart, S. T. Wereley, and J. G. Santiago, A PIV Algorithm for Estimating Time-Averaged Velocity Fields, Journal of Fluids Engineering 122, 285 (2000). - p.58.

[56] F. Scarano, Tomographic PIV: principles and practice, Measurement Science and Technology 24, 012001 (2012). - p.58.

[57] G. E. Elsinga, F. Scarano, B. Wieneke, and B. W. Van Oudheusden, Tomographic particle image velocimetry, Experiments in Fluids 41, 933 (2006). - p.58.

[58] A. Casadellà, O. Schaetzle, K. Nijmeijer, and K. Loos, Polymer inclusion membranes (PIM) for the recovery of potassium in the presence of competitive cations, Polymers 8, 1 (2016). - p.59.

[59] J. H. Choi, J. S. Park, and S. H. Moon, Direct measurement of concentration distribution within the boundary layer of an ion-exchange membrane, , Journal of Colloid and Interface Science 251, 311 (2002). — p.59.

[60] V. Shaposhnik, V. Vasil'eva, and D. Praslov, Concentration fields of solutions under electrodialysis with ion-exchange membranes, Journal of Membrane Science 101, 23 (1995). - p.59.

[61] V. A. Shaposhnik, V. I. Vasil'eva, and O. V. Grigorchuk, The interferometric investigations of electromembrane processes., Advances in colloid and interface science 139, 74 (2008). - p.59.

[62] F. A. Settle, Handbook of Instrumental Techniques for Analytical Chemistry (Prentice Hall, 1997), p. 1024. — p.59.

[63] V. Fleury, J.-N. Chazalviel, and M. Rosso, Coupling of drift, diffusion, and electroconvection, in the vicinity of growing electrodeposits, Physical Review E 48, 1279 (1993). - p.59.

[64] F. C. Leinweber and U. Tallarek, Nonequilibrium electrokinetic effects in beds of ion-permselective particles, Langmuir : the ACS journal of surfaces and colloids 20, 11637 (2004). - p.59, 60.

[65] G. Yossifon and H.-C. Chang, Selection of Nonequilibrium Overlimiting Currents: Universal Depletion Layer Formation Dynamics and Vortex Instability, Physical Review Letters 101, 254501 (2008). — p.59, 60.

[66] J. R. Lakowicz, Principles of fluorescence spectroscopy, 3rd ed. (Springer, 2013). — p.59, 60, 61, 62 .

[67] J. Choi, K. Huh, D. J. Moon, H. Lee, S. Y. Son, K. Kim, H.-Y. H. C. Kim, J.-H. Chae, G. Y. Sung, H.-Y. H. C. Kim, J. W. Hong, and S. J. Kim, Selective preconcentration and online collection of charged molecules using ion concentration polarization, RSC Adv. 5, 66178 (2015). — p.60.

[68] N. Panchuk-Voloshina, R. P. Haugland, J. Bishop-Stewart, M. K. Bhalgat, 
P. J. Millard, F. Mao, W. Y. Leung, and R. P. Haugland, Alexa dyes, a series of new fluorescent dyes that yield exceptionally bright, photostable conjugates., The journal of histochemistry and cytochemistry : official journal of the Histochemistry Society 47, 1179 (1999). — p.60.

[69] P. Roudot, C. Kervrann, and F. Waharte, Lifetime estimation of moving vesicles in frequency-domain fluorescence lifetime imaging microscopy, 2012 9th IEEE International Symposium on Biomedical Imaging (ISBI) 2012, 668 (2012). - p.61, 62.

[70] B. Hötzer, R. Ivanov, P. Bauer, and G. Jung, Investigation of copper homeostasis in plant cells by fluorescence lifetime imaging microscopy, Plant Signaling \& Behavior 7, 521 (2012). - p..

[71] C. D. Geddes, K. Apperson, J. Karolin, and D. J. S. Birch, Chloride sensitive probes for biological applications, Dyes and Pigments 48, 227 (2001). p.61.

[72] E. Karatay, P. A. Tsai, and R. G. H. Lammertink, Rate of gas absorption on a slippery bubble mattress, Soft Matter 9, 11098 (2013). — p.61, 62.

[73] J. Hohlbein, M. Steinhart, C. Schiene-Fischer, A. Benda, M. Hof, and C. G. Hübner, Confined diffusion in ordered nanoporous alumina membranes, Small 3, 380 (2007). — p.61.

[74] FLIM, LI-FLIM, LIFA Software Manual (Lambert Instruments, Roden, 2014), p. 93. - p.62.

[75] G. Scalia and F. Scheffold, Lifetime of fluorescent dye molecules in dense aqueous suspensions of polystyrene nanoparticles, Optics Express 23, 29342 (2015). - p.62. 


\section{CHAPTER 3}

\section{Dynamics of Micro-vortices Induced by lon Concentration Polarization}

This chapter describes the coupled dynamics of the local hydrodynamics and global electric response of an electrodialysis system, which consists of an electrolyte solution adjacent to a charge selective membrane under electric forcing. Under a DC electric current, counter-ions transport through the charged membrane while the passage of co-ions is restricted, thereby developing ion concentration polarization (ICP) or gradients. At sufficiently large currents, simultaneous measurements of voltage drop and flow field reveal several distinct dynamic regimes. Initially, the electrodialysis system displays a steady Ohmic voltage difference $\left(\Delta V_{\text {ohm }}\right)$, followed by a constant voltage jump $\left(\Delta V_{c}\right)$. Immediately after this voltage increase, micro-vortices set in and grow both in size and speed with time. After this growth, the resultant voltage levels off around a fixed value. The average vortex size and speed stabilize as well, while the individual vortices become unsteady and dynamic. These quantitative results reveal that microvortices set in with an excess voltage drop (above $\Delta V_{o h m}+\Delta V_{c}$ ) and sustain an approximately constant electrical conductivity, destroying the initial ICP and having significantly low viscous dissipation.

This chapter is based on:

Dynamics of micro-vortices induced by ion concentration polarization

Joeri C. de Valença, R. Martijn Wagterveld, Rob G. H. Lammertink, Peichun Amy Tsai, Physical Review E Rapid Communications 92, 031003(R) (2015) 


\subsection{Introduction}

Ion concentration gradients emerge during a separation process involving a charge selective surface (electrode or membrane) in an electrolyte solution, inducing a decreasing ion concentration towards the interface and thereby hampering ion transport. For example, for water purification using electrodialysis under electric forcing, the charge selectivity of an ion exchange membrane causes an enrichment of counter-ions on the permeated side of membrane and a depletion of co-ions on the feed side. This so-called ion concentration polarization (ICP), with a decreasing ion concentration on the (feed side) interface, is a common theme in electrochemical applications that influence the performance of ion separation and transport. The diversity of ICP-associated applications has recently motivated numerous studies, ranging from micro-and-nano-junctions [1-8], electrodialysis [9-13], desalination [14, 15], electrodeposition [16-19], and fuel cells [20].

In electrodialysis, theoretical analyses of low dimensions reveal that ICP can drive hydrodynamic instability through an equilibrium [21] or non-equilibrium (electro-osmotic or bulk electro-convective) mechanism [9, 22-27], suggesting an additional charge transport due to ICP-induced fluid motion under a sufficiently large DC voltage. Recent advances have been made with direct numerical simulations (DNS) [28-30], providing insights into ion concentrations and flow velocity adjacent to a charged membrane. Experimentally, under a pressure-driven microchannel flow, the advection and height selection of the unidirectional sheared vortices were characterized along the membrane [11], but the internal vortex structure was not probed. Furthermore, quantitative experiments of the flow field in electrodialysis without shear flow are still missing, in particular under high electrical forcing [9]. This Chapter presents quantitative measurements of the coupled hydrodynamics and electrical response of an ionic solution in the vicinity of a charge selective membrane, under a constant DC electrical current without an external imposed shear flow.

\subsection{Material and methods}

Figure 3.1 shows the experimental setup and the resulting fluid dynamics at high electric forcing in electrodialysis. A cation exchange membrane, CEM (Neosepta CMX, surface area of $3 \mathrm{~mm} \times 4 \mathrm{~mm}$ with thickness of $170 \mu \mathrm{m}$ ), is placed horizontally in a transparent PMMA cell filled with a $10 \mathrm{mM} \mathrm{CuSO}_{4}$ electrolyte between two copper electrodes. Chronopotentiometric measurements are performed between the top cathode and the bottom anode with an potentiostat (Autolab PGSTAT30 Potentiostat). This method consists of forcing a constant DC electric current, $I$, through the ionic solution across the membrane and measuring 


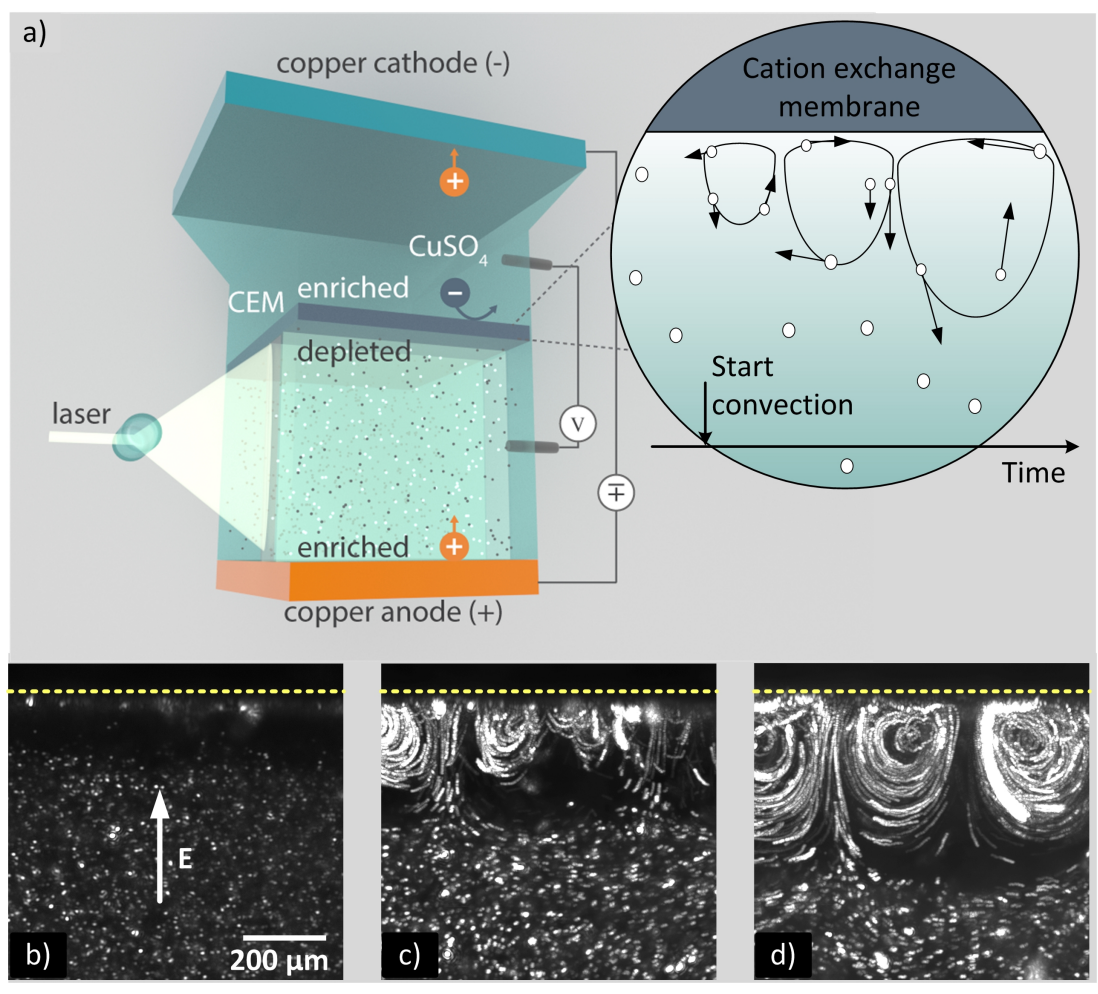

Figure 3.1: (a) Schematic diagram of the experimental setup of a charge selective (cation exchange) membrane, which allows cations to pass the membrane while rejecting anions, immersed in an ion solution of $\mathrm{CuSO}_{4}$ under an electric DC current between two copper electrodes. We simultaneously measure the global voltage drop, $\Delta V$, under a constant applied current, $I$, and local hydrodynamics of the ionic solution. (b-d) Representative motions of the seeding micro-particles reveal different hydrodynamic states in time: (b) Electrical conduction regime without hydrodynamic convection (at $100 \mathrm{~s}$ ). This transforms to a convection regime with micro-vortices growing in size with time, (c-d) $220 \mathrm{~s}$ and $420 \mathrm{~s}$, respectively. The applied DC current density, $i$, was $10 \mathrm{~A} / \mathrm{m}^{2}$. The dashed yellow lines indicate the bottom boundary of the membrane.

the time-dependent voltage difference, $\Delta V(t)$ between the electrodes. The bottom anode, where copper oxidises, serves as a cation source; the top cathode, where copper reduces, acts as a cation sink. This configuration of the electrodes can suppress the occurrence of gravitational convection caused by a variation of fluid density due to ion-concentration since a heavier $\mathrm{Cu}^{2+}$-rich solution is present close to the anode at the bottom of the fluid cell [31]. It was noticed that $\mathrm{Cu}$ dendrites form on the cathode after long experimental runs ( $\geq 1000 \mathrm{~s})$ and 
at a high current density. Thus, electrodes are cleaned before each experiment and the the time-series data has a focus on the time before the maximal ICP condition for the cathode

In addition to the electrical measurements $\Delta V(t)$, simultaneously, the flow motion is observed close to the membrane interface under different DC currents (see figure 3.1b-d). The flow motions are observed by seeding $0.1 \mathrm{wt} \% 5 \mu \mathrm{m}$ polystyrene tracer particles (Microparticles $\mathrm{GmbH}$, with the particle density of $1.05 \mathrm{~g} / \mathrm{cm}^{3}$ ) to the solution. For these nearly buoyancy-neutral micro-particles, the theoretical sedimentation speed is small $(\approx 0.7 \mu \mathrm{m} / \mathrm{s}$, estimated by the Stokes drag equation [32]). The zeta potential of these micro-particles is measured to be $\approx-1 \mathrm{mV}$ for $1 \mathrm{mM} \mathrm{CuSO}_{4}$ (Zetasizer Nano ZS, Malvern). The added microparticles do not influence the electrical response of the electrolyte solution, as shown by similar $\Delta V(t)$ data obtained with and without the micro-particles. To avoid particle aggregation, a non-ionic surfactant, Tween $80,(0.1 w t \%)$ is added to the solution. Flow fields are obtained employing a particle image velocimetry (PIV) technique $[32,33]$. The micro-particles are illuminated by a thin laser sheet (Firefly laser, $808 \mathrm{~nm}$, Oxford Lasers) with a pulse duration of $20 \mu s$ and a pulse power of $0.3 \mathrm{~mJ} /$ pulse (illuminating $3 \mathrm{~mm} \times 2 \mathrm{~mm} \times 200 \mu \mathrm{m}$ ). The scattered light is captured at $20 \mathrm{~Hz}$ by a CCD camera (Sony XCG-H280E, $1920 \times 1080 \mathrm{px}^{2}$ ), with a magnifying lens (Navitar, 2-14 $\times$ ) placed perpendicular to the laser sheet. Sets of 50-200 images are analysed using ImageJ software (NIH) to visualize and measure the vortex motions and sizes.

\subsubsection{Particle Image Velocimetry Analysis}

The vortex speed and size is also determined using PIV analysis. Each particle movie needs its own parameters to get reliable vector fields. The most important are the correlation window size and the vector post-processing. The steps to get accurate vector fields for the different data sets used in this thesis are discussed below. Unless stated otherwise the time difference between the images is $\Delta t=$ $0.1 \mathrm{~s}$.

Recording of images The flow is visualized by capturing images of seeding micro-particles, with a frame rate of $20 \mathrm{~Hz}$ for about 1000-2000 s. Depending on the particle velocity and magnification, an appropriate $\Delta t$ for the PIV calculation is chosen, ranging from $0.1 \mathrm{~s}$ to $0.5 \mathrm{~s}$. To image the full anode compartment a magnification of $M=2.5$ is used, this has a resolution of 1 pixel $=2.0 \mu \mathrm{m}$. To get more detail, like in figure 3.3, a higher magnification is used, $M=5.7$, with resolution of 1 pixel $=0.87 \mu \mathrm{m}$. The focal depth of the optical system at this magnification is measured to be $\approx 200 \mu \mathrm{m}$. Particles outside this depth of field are larger, have a lower light intensity, and are systematically filtered in the PIV 
analysis.

Image preprocessing Image preprocessing was used to increase the signalto-noise ratio in the PIV calculations. The particle to background contrast in the raw images is enhanced with a build-in feature in the DaVis software, called the local min/max filter. First the local background intensity of each pixel is removed by subtracting the local minimum in a window of $5 \times 5$ pixels (sliding minimum subtraction). From the raw images, the ratio between the local and global maximum is determined, by dividing the global sliding maximum (50 pixel window) by the local sliding maximum ( 5 pixel window). Finally these two images are multiplied (background subtraction $\times$ ratio global to local maximum) to get the normalized particle intensity. With this method, particle intensity variations between images are smoothed, and bright aggregates become less dominant for the cross correlation method. A geometrical mask covers the membrane and/or channel wall so no vector field is calculated in that region.

Multigrid vector calculation To calculate the flow field, a multigrid cross correlation method with decreasing window size is used. The first, larger, interrogation window determines a reference vector field. This field is then used to calculate a window shift of the next correlation with smaller interrogation window $[34,35]$. This shift ensures the same particles are correlated with each other. The first interrogation window is $128 \times 128$ pixel $^{2}$ and the second $96 \times 96$ pixel $^{2}$. The vectors are displayed with a $50 \%$ overlap, meaning each vector represents an area of $48 \times 48$ pixels $\left(42 \times 42 \mu \mathrm{m}^{2}\right.$ for the current density of $\left.9 \mathrm{~A} / \mathrm{m}^{2}\right)$.

Vector post-processing To eliminate erroneous vector a LaVision vector post-processing algorithm is used. Outlier detection is used, based on the median value of the nearest neighbours. This value is less sensitive to neighbouring outliers with large values compared to a normal average. If appropriate, a new vector is calculated, and groups of erroneous vectors can also be excluded. This scheme consists of the four following steps. First, vectors outside the allowed deviation from the neighbours are eliminated, with the criterion below:

$$
\begin{aligned}
& U_{\text {median }}-a \cdot U_{r m s} \leq U \leq U_{\text {median }}+a \cdot U_{r m s} \\
& U_{r m s}=\sqrt{\frac{1}{n} \sum_{i=1}^{n}\left(U_{i}-U_{\text {median }}\right)^{2}}
\end{aligned}
$$

where $a=2$. This criterion is used for both $x$ and $y$ components of a planar velocity vector $(u, v)$ to check the validity of the velocity vector. In the second step, all vectors with less then three neighbour vectors are also removed. In the third step, new vectors for the empty vector positions are found with an iterative process. For vectors with three or more neighbours, the median criteria is checked 
with $a=3$. If the vector is identified as false, a lower correlation peak is checked for fitting up to the 4 th lowest peak. Once no more vectors can be added, the final step effectively detects and subsequently removes small groups of spurious vectors (with less than four vectors) which have not been detected in the steps 1 and 2 .

If no good vectors are found in the integration windows, the vectors in the empty spaces are interpolated or extrapolated. The average value of the $x$ and $y$ vector components of the non zero nearest neighbours is determined and subsequently used. This is done iteratively to fill up the whole grid.

Temporal averaging The dynamics are slow, so for five consecutive vector fields the orthogonal components are averaged separately (sliding average). Final vector fields thus represent the average motion during $\Delta t=0.5 \mathrm{~s}$, case of $10 \mathrm{~A} / \mathrm{m}^{2}$. For lower current densities the dynamics is slower so one vector field is calculated from two images with $\Delta t=0.5 \mathrm{~s}$. The sliding average will represent the motion during $\Delta t=2.5 \mathrm{~s}$. In addition, the vorticity of the vector field $\left(\partial v_{y} / \partial x-\partial v_{x} / \partial y\right)$ is calculated using a finite central difference with the nearest neighbours. Subsequently, this field is smoothed and displayed as a colour gradient, see figure 3.3.

Determining the vortex size and speed The vortex size (or mixing layer thickness), $L_{m i x}$, and speed (root mean square velocity in the vortex area) are determined from the final vector fields. The vortex boundary is characterized by the sharp decay of the vertical velocity component, $v_{y}$, away from the membrane. From a horizontal row (40 vectors) the average value is taken. These average values are interpolated with a cubic spline to get a smooth function representing the change in vertical velocity. The vortex region is quantified by the distance between the membrane position (determined with ImageJ) and position where the vertical velocity drops below a fixed threshold value. For each current density, the threshold value is chosen so the PIV vortex size matches the ones observed in the stacked images. The absolute value of the vectors in this vortex region is averaged to obtain the vortex speed.

\subsection{Results and discussion}

Each experiment starts with a uniform concentration of $\mathrm{CuSO}_{4}$ at both sides of the membrane. As a DC electric current is forced through the charged membrane, the counter-ions $\left(\mathrm{Cu}^{2+}\right.$ cations), which can easily pass through the membrane, migrate upwards, whereas co-ions $\left(\mathrm{SO}_{4}{ }^{2-}\right.$ anions) migrate downwards. Because the co-ions are retained by the charged membrane, the co-ion concentration enriches at the upper (cathode) side and depletes at the bottom (anode) side of 
the membrane. At a critical condition, a maximum ion-depletion occurs, with a vanishing co-ion concentration at the membrane surface. The maximum linear gradient in which this is possible is called the limiting current condition. The limiting current density can be estimated by balancing electromigration with diffusion of the co-ions $[10,36]$ :

$$
i_{\text {lim }}=\frac{z_{+} F D}{\left(\overline{t_{+}}-t_{+}\right)} \frac{c_{\delta}}{\delta}
$$

The anions cannot leave the system, because both the membrane and anode are cation selective. The maximum linear gradient will therefore have $c_{m}=0$ at the membrane and $c_{\delta}=2 \cdot c_{0}$ at the anode. The distance between anode and membrane of $\delta=2 \mathrm{~mm}$.

The membrane transport number is estimated from chronopotentiometry, see figure 2.5. It was found that $\overline{t_{+}}=0.96$, while $t_{+}=0.4$ [37]. Using $D=$ $8.55 \cdot 10^{-10} \mathrm{~m}^{2} / \mathrm{s}[38], F=96480 \mathrm{C} / \mathrm{mol}$ and $z_{+}=2$ results in:

$$
i_{l i m}=2.9 \mathrm{~A} / \mathrm{m}^{2}
$$

This value was also experimentally confirmed using five consecutive voltage sweeps with $10 \mathrm{mV} / \mathrm{s}$ from $0-3 \mathrm{~V}$.

\subsubsection{Chronopotentiometric response of overlimiting current}

Figure 3.3 shows the experimental results of the global electrical responses of the electrodialysis system. In time, the redox reactions at the electrodes start immediately, and ion concentration polarization is gradually developed. The initial constant voltages $\left(\Delta V_{\text {ohm }}\right)$ reflects a constant Ohmic resistance of the electrodialysis system: $R=11.2 \mathrm{k} \Omega$, obtained from a linear fit of $\Delta V_{o h m}$ for different $I$. This value corresponds to the expected resistance based on the conductivity of $10 \mathrm{mM} \mathrm{CuSO}_{4}$ in the geometry of the system, see section 2.1.2 and 2.1.1.

Subsequently, ions are depleted close to the anode side of the membrane. The depletion grows until the critical limiting condition, which leads to an increased electrical resistance. This critical condition is manifested by a sharp increase in measured voltage, $\Delta V_{c}$, after a transitional time $\tau_{c}$. By the same token for $i_{\text {lim }}$ described above, $\tau_{c}$ can be estimated using Fick's second law with a vanishing co-ion concentration $\left(c_{-}\right)$at the membrane surface, i.e., Sand's equation $[10,36]$ :

$$
\tau_{\mathrm{s}}=\frac{\pi D}{4}\left(\frac{c_{0} z F}{\overline{t_{+}}-t_{+}}\right)^{2} \frac{1}{i^{2}}
$$

In agreement with the theory, our $\tau_{c}$ has a linear relationship with $1 / I^{2}$, with a fitted cation transport number in the membrane of 0.96 , see figure 2.5 . This is 
consistent with the previous experimental results [10, 36, 39]. The voltage jump $\Delta V_{c}$ corresponds to the (electrical current) plateau region in the $I V$ curve under a DC voltage (e.g. figure 4 in Ref. [36]) and depends on the type of membrane. We measured $\Delta V_{c}=0.3 \pm 0.03 V$ for different $i$, ranging from 5.6 to $15.7 \mathrm{~A} / \mathrm{m}^{2}$. The jump is followed by a further voltage increase, $\Delta V_{\text {olc }}$, until $\Delta V$ reaches a saturated value at a later time.

The first two characteristic electric responses, $\Delta V_{\text {ohm }}$ and the onset of $\Delta V_{c}$, are well understood, however the later-time $\Delta V_{\text {olc }}$ and the transport mechanisms causing overlimiting conductance, beyond $\Delta V_{c}$, have been extensively debated [15]. This phenomena is manifested in the additional voltage drop $\Delta V_{\text {olc }}$ observed under a constant $I$ or the increasing currents in the conventional currentvoltage curves under DC voltages by other studies [36, 40]. Several mechanisms have been proposed, including water dissociation, hydrodynamic convection, and charge-induced membrane discharge [10, 15, 21, 22, 26, 41, 42]. Previous work has shown that water dissociation for our type of CMX membrane is insufficient to account for the observed overlimiting conductance $[15,36,40]$. To gain insight, the coupled hydrodynamics is analysed from the captured images, using particle pathlines (figure $3.1 \mathrm{~b}-\mathrm{d}$ ) and PIV analysis (figure $3.3 \mathrm{~b}-\mathrm{d}$ ).

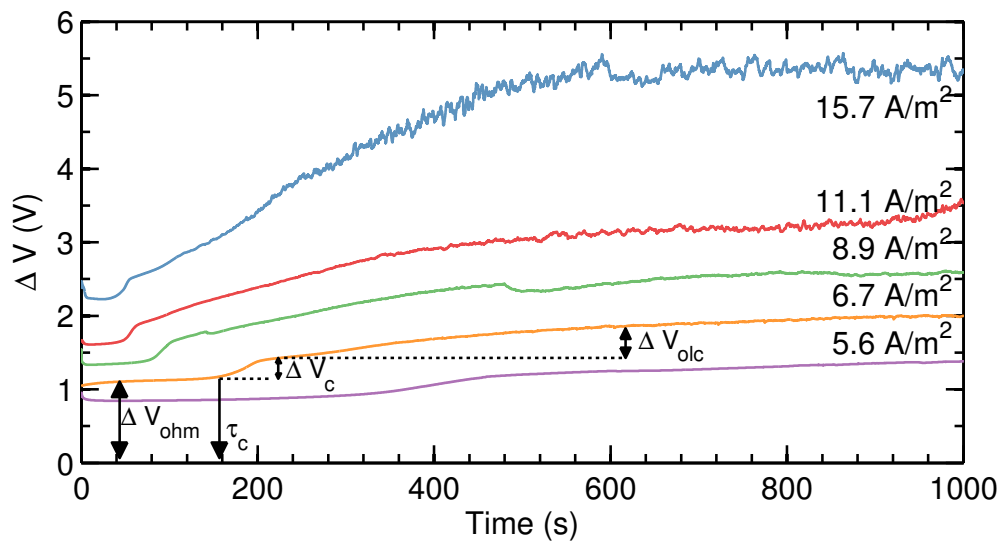

Figure 3.2: Chronopotentiometric measurements of the total electric voltage drop, $\Delta V(t)$ changing in time across the cell, under different applied DC current densities, $i$, through the charge selective membrane. The total potential drop reveals three distinct responses: (1) The initial constant Ohmic resistance of the whole cell $\Delta V_{\text {ohm }}=I R_{\text {ohm }}$, (2) The critical voltage jump, $\Delta V_{c}$ that starts at the transitional time, $\tau_{c}$, and (3) The overlimiting voltage drop over the vortex mixing region, $\Delta V_{\text {olc }}$. 


\subsubsection{Dynamics of micro-vortices during overlimiting current}

In figure 3.3, a representative set of the coupled dynamics of electric response $\Delta V(t)$ and vertical vortex size $L_{m i x}$ (measured from the membrane surface) is shown. The error bars represent the variation of the individual vortex sizes. Four distinct regimes are delineated in figure 3.3. The initial Regime I is electric Ohmic conductive, with no hydrodynamic convection observed from the motion of the micro-particles. In Regime II, voltage jump $\Delta V_{c}$ occurs, with an increasing electrical resistance of the electrodialysis cell. This starts at the transition time (e.g., $\tau_{c} \approx 77 \mathrm{~s}$ for $i=9 \mathrm{~A} / \mathrm{m}^{2}$ ). In Regime III, small counter-rotating vortex pairs ap-

(a)

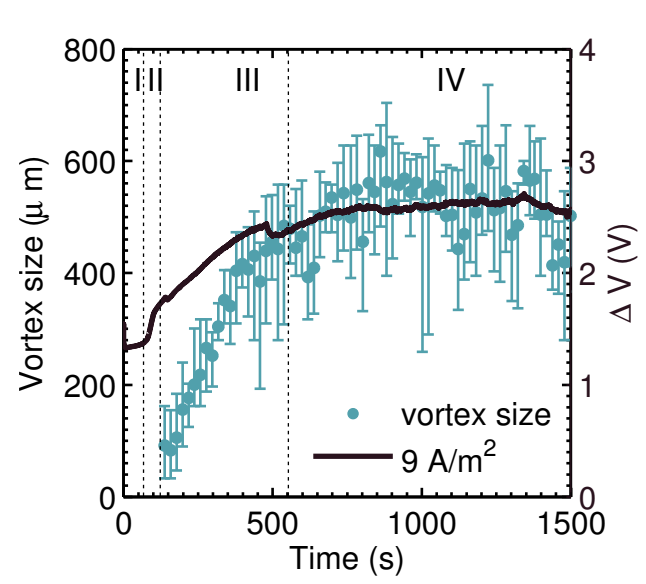

(b)
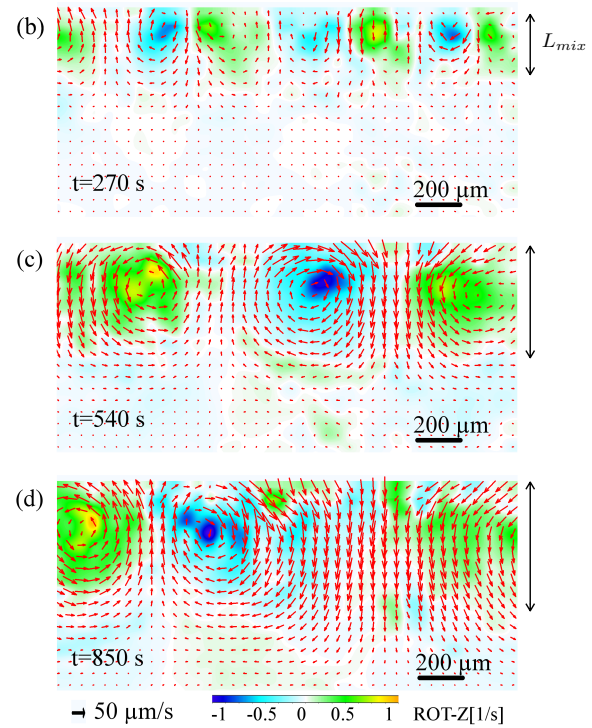

Figure 3.3: (a) Simultaneous measurements of the dynamics of vertical micro-vortex size $(\bullet)$ and voltage response $\Delta V(-)$, under a constant current density of $9 \mathrm{~A} / \mathrm{m}^{2}$ across the charge selective membrane. Four characteristic transport regimes are delineated by the dashed lines. I. electric Ohmic conduction without hydrodynamic convection, II. a potential jump with the development of ICP, III. a hydrodynamic convection regime with linearly growing electric resistance and vortex size in time, and IV. a saturated regime with saturated values of both vortex size and electric resistance. (b-d) The corresponding flow field, velocity vectors and vorticity $(\nabla \times \vec{u})$ obtained with a PIV technique at different times; (b) and (c) show the growth and (d) the unsteady nature of the micro-vortices. The vertical arrow in (b-d) indicates the length scale of vortex mixing layer, $L_{m i x}$, which initially increases with time. $L_{m i x}$ is measured to be 260 , 480 , and $550 \mu \mathrm{m}$ from (b) to (d), respectively, at different times $(t)$ indicated. 
pear along the membrane surface. The thickness of this mixing vortex layer $L_{m i x}$ grows in time (e.g. figure $3.3 \mathrm{~b}$ ). Simultaneously, $\Delta V(t)$ gradually grows in this regime. Finally, in Regime IV, both $\Delta V$ and vortex size saturate and fluctuate at fixed values. The vortices are observed to move laterally and merge together, showing unsteady dynamics (see the supporting videos [43]). Consistent with these experimental findings, the unsteady and chaotic movements of saturated vortexes have been observed in recent direct numerical simulations, where instead of a constant current, a constant voltage drop is the control parameter and the current fluctuates around a saturated value [29, 30].

In this experimental data micro-vortices only set in from Regime III, slowly growing in size and speed with time, accompanied by a linear increase of $\Delta V$ in time (e.g. $150-450 \mathrm{~s}$ in figure 3.3a). In addition, the experimental result of rms vortex velocity is quantitatively consistent with that found in $2 \mathrm{D}$ simulations of electro-osmotic instability under similar electrical forcing (shown in figure 4c in Ref. [9]), albeit different electrical boundary conditions (constant current vs. constant voltage). Based on these observations, the convective transport carried by swirling micro-vortexes is very likely the main cause of the overlimiting conductance observed. However, the fundamental origin of the convective instability, which can be induced via an equilibrium or non-equilibrium mechanism as suggested by different theories $[15,21,22,26]$, remains elusive. This data reveals the quantitative growth of $\Delta V_{\text {olc }}$ and vortex speed and size and can motivate future theoretical investigations under constant currents to identify the primary origin of the convective instability.

Figure 3.4 shows the dependence of electroconvective dynamics on the forced current density. In Regime III, both the voltage $\Delta V_{\text {olc }}$ and the mixing layer thickness of vortex region $L_{m i x}$ initially grow linearly (see figure 3.3a). These growth rates versus applied current densities are shown in Figure 3.4a. Figure 3.4b displays the average vortex speed and size in the saturated, overlimiting Regime IV, where unsteady dynamics are observed. Both vortex size and speed increase with current density, but the rms velocity increases stronger than the vortex size for the increasing current, underlining the importance of convective transport in this regime. The large error bars at the higher current densities reflect the increasing fluctuations and chaotic motions of the individual vortices and not the reproducibility of the experiments.

From the data of growth rates in figure $3.4 \mathrm{a}$, the voltage difference required to grow to a vortex region $L_{m i x}$ can be calculated using Regime III data: $\Delta V_{\text {olc }}^{I I I}=$ $(\delta(\Delta V) / \delta t)\left(\delta L_{m i x} / \delta t\right)^{-1} L_{m i x}$. Revealed in figure $3.4 \mathrm{c}$, this voltage difference estimated for the final saturated vortex region agrees well with the voltage drop $\Delta V_{\text {olc }}$ over the saturated mixing layers observed in Regime IV: $\Delta V_{\text {olc }}^{I V}=$ $\Delta V-I R_{o h m}-\Delta V_{c}$, indicating that micro-vortices only set in with an excess volt- 

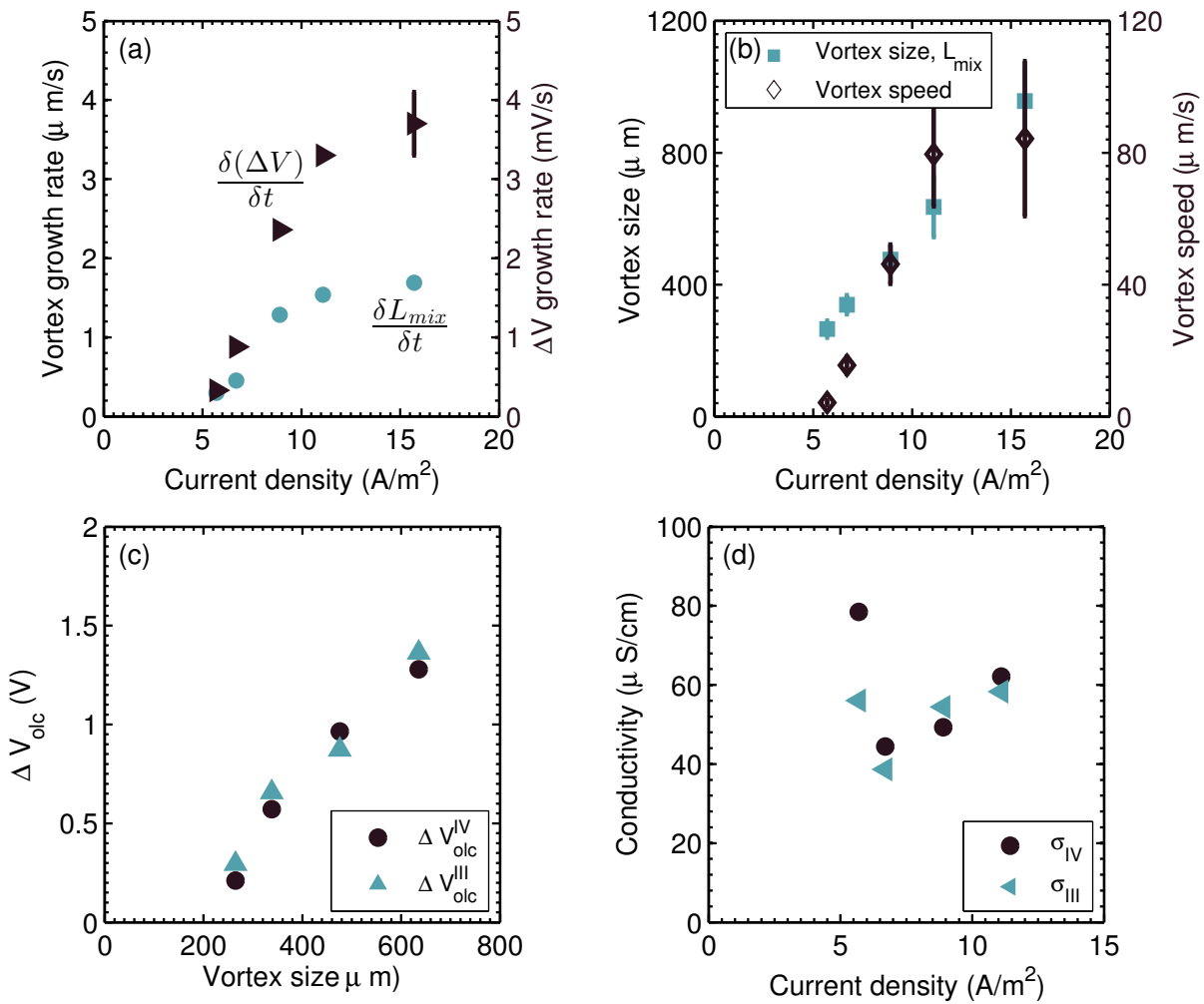

Figure 3.4: (a) The growth rate of vortex size $(\bullet)$ and voltage drop $\Delta V(\bullet)$ during the linear convective Regime III indicated in figure 3, under different current densities. (b) The dependence of the average vortex size $\left(L_{m i x}\right)(\square)$ and speed $(\diamond)$ on the applied current density across the membrane in the saturated convective regime IV. The error bar shows the standard deviation of the time averaged values, revealing more fluctuations in vortex dynamics at higher currents. (c) The average voltage drops, $\Delta V_{\text {olc }}$, over the saturated vortex size in Regime IV,$(\bullet)$ obtained from the growth rate data of Regime III ( $\Delta$ ) and from the data of saturated $\Delta V_{\text {olc }}^{I V}$ in Regime IV $(\bullet)$. (d) The average conductivity in the mixing layer in the growth regime, $\sigma_{\text {III }}(\triangleleft)$, and in the saturated regime $\sigma_{\mathrm{IV}}(\bullet)$.

age drop, $\Delta V_{\text {olc }}$, across the mixing layer of vortex region. Furthermore, one could estimate the electrical conductivity $\sigma$ in this mixing region due to the presence of swirling vortices: $\sigma_{\mathrm{IV}}=L_{m i x} i / \Delta V_{o l c}^{I V}$, for each $i$. This conductivity is approximately constant for different current densities, and similar to the conductivity of the mixing layer in the growth regime III: $\sigma_{\text {III }}=i\left(\delta L_{m i x} / \delta t\right)(\delta(\Delta V) / \delta t)^{-1}$, revealed in figure $3.4 \mathrm{~d}$. We found that the conductivity in the mixing layer is sim- 
ilar for all the experiments: $\sigma=55 \pm 12 \mu \mathrm{S} / \mathrm{cm}$. In contrast, in the under-limiting and limiting regimes, without vortices, ion concentrations are slowly depleted due to electrical migration and concentration diffusion, as well as the charge selectivity of the membrane. In the overlimiting regime, micro-vortices are initiated, continue to grow, and finally are saturated, right below the membrane.

\subsubsection{Viscous dissipation within micro-vortices}

The vector velocity field quantifies the shear stresses in the fluid. Through friction energy can be dissipated, called viscous dissipation. This can be calculated from the velocity gradients via [44]:

$$
P_{v i s c}=\mu \int\left[\left(\frac{\partial v_{x}}{\partial x}\right)^{2}+\left(\frac{\partial v_{x}}{\partial y}\right)^{2}+\left(\frac{\partial v_{y}}{\partial x}\right)^{2}+\left(\frac{\partial v_{y}}{\partial y}\right)^{2}\right] d V
$$

where $v_{x}=d x / d t$ and $v_{y}=d y / d t$, volume element $d V$, and $\mu$ the liquid dynamic viscosity $(1 \mathrm{mPa} \cdot \mathrm{s})$.

The planar velocity field has four different gradients at each vector points. The gradients are calculated with a nearest neighbour approach. At the boundary only the inner neighbour is used to calculate the gradient. The four gradients are squared and added to get the viscous dissipation for each vector (grid) point. The shear perpendicular to the observation plane (in the $\mathrm{z}$ direction) cannot be calculated and is not included in the volume integral of viscous dissipation.

To show the dependence of viscous dissipation on the distance away from the membrane surface the viscous dissipation is averaged in a horizontal layer. This value is assumed to be the dissipation in the whole horizontal layer (of $42 \mu \mathrm{m}$ thickness) with the membrane area of $13.5 \mathrm{~mm}^{2}$. Figure 3.5 shows the estimated viscous dissipations of micro-vortices (between 200 and $500 \mathrm{~s}$ ) under different dc currents. The error bar displays the standard deviation from the horizontal averaging in one vector field and the time averaging of regime IV (200-500 s). The total viscous dissipation in the anode compartment is given in Table 3.1.

Since the voltage drop over the mixing region is measured the electrical input power can be calculated via:

$$
P_{\text {elec }}=i A_{\text {mem }} \Delta V_{\text {olc }}
$$

The current density, $i$, is fixed and the membrane area, $A_{m e m}=13.5 \mathrm{~mm}^{2}$. From the chronopotentiometric responses (figure 3.3) the overlimiting voltage drop over the mixing layer is determined: $\Delta V_{\text {olc }}=\Delta V_{\text {total }}-I R_{\text {ohmic }}-\Delta V_{c}$.

The ratio viscous dissipation to electrical input power $\left(P_{v i s c} / P_{i n}\right)$ is estimated to be $10^{-8}-10^{-9}$. Increasing the current, increases the voltage over, size of and 


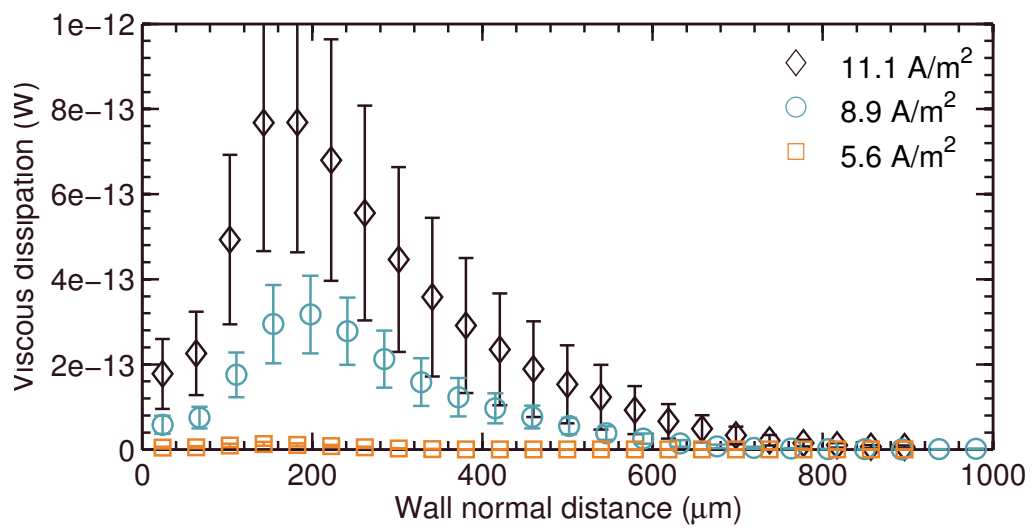

Figure 3.5: Viscous dissipations of micro-vortices, depending on the vertical distance away from the membrane surface, for three different dc electrical currents.

speed inside the vortices and the ratio $P_{v i s c} / P_{i n}$. Note that this viscous ratio is very likely to be underestimated since a no-shear condition is used to estimate the viscous dissipation in $\mathrm{z}$ direction. Still this indicates extremely low power of energy dissipation by convective vortices, and thus $\Delta V_{\text {olc }}$ represents the electrical resistance of the mixing layer of low ion concentration. The advection flow modifies the anion concentration gradient by bringing anions towards the membrane, which impairs the early-time ICP (in Regime II) and sustains overlimiting conductivity.

\begin{tabular}{|l|l|l|l|l|}
\hline & $P_{\text {visc }}[\mathrm{W}]$ & $P_{\text {in }}[\mathrm{W}]$ & $P_{\text {visc }} / P_{\text {in }}$ & $\Delta V_{\text {olc }}(\mathrm{V})$ \\
\hline $5.6 \mathrm{~A} / \mathrm{m}^{2}$ & $6.2 \cdot 10^{-14}$ & $1.9 \cdot 10^{-5}$ & $3.3 \cdot 10^{-9}$ & 0.21 \\
\hline $8.9 \mathrm{~A} / \mathrm{m}^{2}$ & $2.0 \cdot 10^{-12}$ & $1.1 \cdot 10^{-4}$ & $1.8 \cdot 10^{-8}$ & 0.96 \\
\hline $11.1 \mathrm{~A} / \mathrm{m}^{2}$ & $5.7 \cdot 10^{-12}$ & $2.0 \cdot 10^{-4}$ & $2.9 \cdot 10^{-8}$ & 1.28 \\
\hline
\end{tabular}

Table 3.1: Results of viscous dissipation $\left(P_{v i s c}\right)$, electrical input power $\left(P_{i n}\right)$, viscous ratio $\left(P_{\text {visc }} / P_{i n}\right)$ and overlimiting voltage $\left(\Delta V_{\text {olc }}\right)$ at three different dc currents.

\subsection{Conclusion}

In summary, the electrodialysis system involving a charge selective membrane for charge separation in general presents four distinct dynamic regimes observed in chronopotentiometric measurements: (I) a linear Ohmic electric response as charge diffusion and migration takes place; (II) a jump in electrical response 
$(\Delta V)$ during the development of ICP due to charge-selectivity of the membrane; (III) a linear growth regime where micro-vortices grow in both size and speed with time; and finally (IV) a saturated electro-convective regime having saturated values of vortex speed/size and voltage response $\Delta V$. Our quantitative results of the growth rates and saturated electro-convective responses elucidate that micro-vortices only set in with an excess voltage $\Delta V_{\text {olc }}$, have negligible viscous dissipation, and moreover sustain a nearly constant conductivity in the mixing region. 


\section{A Extra experimental details}

The whole setup is fixed on an optical table for vibration isolation. The laser is fixed with a vertical laser sheet. The cell is placed on a six-axis stage with three traverse $(x, y, z)$ and three rotational $(\theta, \varphi, \zeta)$ axis of motion, see figure 3.6. The anode compartment is placed at the height of the laser centre and $\approx 10 \mathrm{~cm}$ from the laser shutter. The alignment is such that cross-section is parallel to the front window and $\approx 1 \mathrm{~mm}$ behind it.
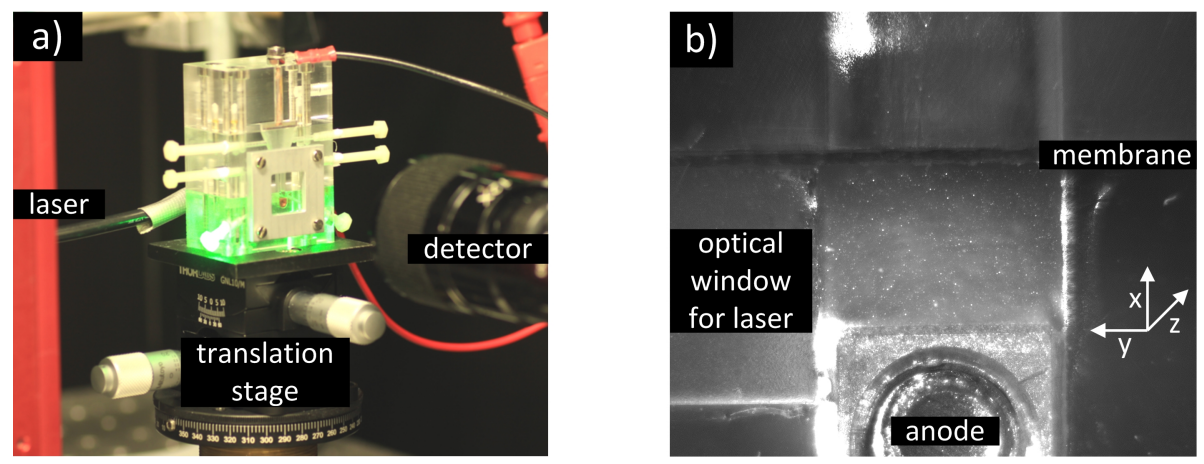

Figure 3.6: A vertical laser sheet illuminates a cross-section of the membrane test cell. The detector (camera with lens) is placed perpendicular to the beam and records the illuminated particles reflection. (a) A picture of the setup. (b) A recorded particle image of the compartment with. The horizontal direction is taken as the $x$ axis since the concentration gradient occurs in this direction.

The camera is placed on a $x y z$ translation stage with the line of sight perpendicular to the laser sheet. The point of focus is aimed to be inside the laser sheet in the illuminated volume. The image recording frame rate is triggered by the internal clock of the laser. The shutter opens before the laser pulse and closes after. Directly afterwards the camera writes the image data to a computer using Streampix software, with a maximum frequency is 32 frames per second. This is the upper limit of the camera.

The electric and optic measurements are synchronized with a LED light regulated by the potentiostat. The lamp is placed behind the cell and indicates when the electrical measurements start and end. This is visible in the optical recordings, allowing accurate synchronization between measurement techniques. 


\section{B Discussion on the error in the vector field analysis}

In the PIV algorithm erroneous vectors are determined and interpolated. Here the nature of the main errors is described and their influence on the final velocity. First the vector evaluations close to the membrane interface are described, second, the resolution limit of the system, and finally, the errors induced by out of plane motion $\left(v_{z}\right)$.

Velocity error at the boundary The membrane interface marks the boundary between the solid and liquid (flow) region. Only part of the interrogation window is filled with moving particles and the motion is often highly non-linear, due to high velocity gradients. This gives falsely give rise to false linear correlations inside the correlation window. Light reflection from the membrane or from sticking particles can also bright and dominant in the correlation calculation. The validity of these boundary vector is also more difficult to check because they have less direct neighbours. A strong filter is used to remove spurious vectors and interpolate the values from the vectors from the central regions. Therefore the high velocity particles at the interface are often neglected.

Optical resolution limit Particles of $d=5 \mu \mathrm{m}$ are used to have a low electrophoretic mobility and strong light scattering. This limits the seeding density and therefore the resolution of the system. Our final interrogation window is $42 \times 42 \mu \mathrm{m}^{2}$. Non-linear motion within this window, e.g. small vortices, cannot be detected with the current PIV algorithm. Therefore image stacks are used to detect small vortices in the initial vortex growth region (region III).

Out of plane motion $\left(v_{z}\right)$ The planar PIV setup traces the in-plane motion $\left(v_{x}\right.$ and $\left.v_{y}\right)$, but sometimes the particle motion clearly has a significant $v_{z}$ component. This is observed if the particle intensity changing in time, indicating particle movement perpendicular to the laser sheet. In addition, particles moving out of the focal plane (with thickness estimated as $\approx 200 \mu \mathrm{m}$ ) with $v_{z}$ produce blurred particle images. Our current experimental setup is for a planar PIV, accurately measuring particle in-plane motions. Evidently the nature of the vortex induced by ICP is three-dimensional. Precise and accurate $z$-component of velocity fields require new experimental equipments and future research effort.

In short, these three different types of errors lead to an underestimation of the real fluid velocities. 


\section{C Supporting movies}

Two supporting movies of figure 3 (imposed dc current density of $9 \mathrm{~A} / \mathrm{m}^{2}$ ) can be downloaded from the website of the journal this work is published in:

http://journals.aps.org/pre/abstract/10.1103/PhysRevE.92.031003

Movie 1 shows the vortex motions within $5 \mathrm{~s}$, made of an overlay of 100 images (ImageJ, Z project, max intensity). The in-plane flow speed can be estimated with the length of the particle pathlines. From the measurements, the initial growth of the vortices is linearly correlated with the resulting voltage difference $\Delta V$. The corresponding voltage response is simultaneously shown in a plot below the overlays, as well as the analysed vortex size from the overlaid images.

Movie 2 displays the flow field (every 5 s) obtained with the PIV analysis. The background colour represents the calculated vorticity. Together with the time evolving velocity vectors, the voltage response and the measured velocities in the vortex area are shown. 



\section{Bibliography}

[1] S. J. Kim, Y. C. Wang, J. H. Lee, H. Jang, and J. Han, Concentration Polarization and Nonlinear Electrokinetic Flow near a Nanofluidic Channel, Phys. Rev. Lett. 99, 044501 (2007). - p.72.

[2] G. Yossifon and H.-C. Chang, Selection of Nonequilibrium Overlimiting Currents: Universal Depletion Layer Formation Dynamics and Vortex Instability, Phys. Rev. Lett. 101, 254501 (2008). - p..

[3] S. J. Kim, S. H. Ko, K. H. Kang, and J. Han, Direct seawater desalination by ion concentration polarization, Nature Nanotechnology 5, 297 (2010). — p..

[4] S. J. Kim, S. H. Ko, R. Kwak, J. D. Posner, K. H. Kang, and J. Han, Multi-vortical flow inducing electrokinetic instability in ion concentration polarization layer, Nanoscale 4, 7406 (2012). - p..

[5] H.-C. Chang, G. Yossifon, and E. A. Demekhin, Nanoscale Electrokinetics and Microvortices: How Microhydrodynamics Affects Nanofluidic Ion Flux, Annu. Rev. Fluid Mech. 44, 401 (2012). - p..

[6] J. Schiffbauer, U. Liel, and G. Yossifon, Concentration dependence of nanochannel impedance and the determination of surface charge, Phys. Rev. E 89, 033017 (2014). - p..

[7] C. P. Nielsen and H. Bruus, Concentration polarization, surface currents, and bulk advection in a microchannel, Phys. Rev. E 90, 043020 (2014). p..

[8] Y. Green, S. Park, and G. Yossifon, Bridging the gap between an isolated nanochannel and a communicating multipore heterogeneous membrane, Phys. Rev. E 91, 011002(R) (2015). - p.72.

[9] S. M. Rubinstein, G. Manukyan, A. Staicu, I. Rubinstein, B. Zaltzman, R. Lammertink, F. Mugele, and M. Wessling, Direct Observation of a Nonequilibrium Electro-Osmotic Instability, Phys. Rev. Lett. 101, 236101 (2008). - p.72, 80 .

[10] V. V. Nikonenko, N. D. Pismenskaya, E. I. Belova, P. Sistat, P. Huguet, G. Pourcelly, and C. Larchet, Intensive current transfer in membrane systems: Modelling, mechanisms and application in electrodialysis, Adv. Colloid Interface Sci. 160, 101 (2010). — p.77, 78.

[11] R. Kwak, V. S. Pham, K. M. Lim, and J. Han, Shear Flow of an Elec- 
trically Charged Fluid by Ion Concentration Polarization: Scaling Laws for Electroconvective Vortices, Phys. Rev. Lett. 110, 114501 (2013). — p.72.

[12] M. Wessling, L. G. Morcillo, and S. Abdu, Nanometer-thick lateral polyelectrolyte micropatterns induce macrosopic electro-osmotic chaotic fluid instabilities, Scientific Reports 4, (2014). - p..

[13] R. abu-Rjal, V. Chinaryan, M. Z. Bazant, I. Rubinstein, and B. Zaltzman, Effect of concentration polarization on permselectivity, Phys. Rev. E 89, 012302 (2014). - p.72.

[14] A. Mani and M. Z. Bazant, Deionization shocks in microstructures, Phys. Rev. E 84, 061504 (2011). - p.72.

[15] V. V. Nikonenko, A. V. Kovalenko, M. K. Urtenov, N. D. Pismenskaya, J. Han, P. Sistat, and G. Pourcelly, Desalination at overlimiting currents: State-of-the-art and perspectives, Desalination 342, 85 (2014). — p.72, 78, 80.

[16] V. Fleury, M. Rosso, J.-N. Chazalviel, and B. Sapoval, Experimental aspects of dense morphology in copper electrodeposition, Phys. Rev. A 44, 6693 (1991). - p.72.

[17] J. M. Huth, H. L. Swinney, W. D. McCormick, A. Kuhn, and F. Argoul, Role of convection in thin-layer electrodeposition., Phys. Rev. E 51, 3444 (1995). - p..

[18] M. Rosso, Electrodeposition from a binary electrolyte: new developments and applications, Electrochim. Acta 53, 250 (2007). - p..

[19] G. González, M. Rosso, E. Chassaing, and J.-N. Chazalviel, Experimental and theoretical study of the onset of the growth of an irregular metal electrodeposit, Electrochimica Acta 53, 141 (2007). — p.72.

[20] K. Scott, W. Taama, S. Kramer, P. Argyropoulos, and K. Sundmacher, Limiting current behaviour of the direct methanol fuel cell, Electrochimica Acta 45, 945 (1999). - p.72.

[21] I. Rubinstein and B. Zaltzman, Equilibrium Electroconvective Instability, Phys. Rev. Lett. 114, 114502 (2015). - p.72, 78, 80.

[22] S. S. Dukhin, Electrokinetic phenomena of the second kind and their applications, Adv. Colloid Interface Sci. 35, 173 (1991). - p.72, 78, 80.

[23] I. Rubinstein, E. Staude, and O. Kedem, Role of the membrane surface in concentration polarization at ion-exchange membrane, Desalination 69, 101 (1988). - p..

[24] I. Rubinstein and B. Zaltzman, Electro-osmotically induced convection at a permselective membrane, Phys. Rev. E 62, 2238 (2000). — p..

[25] F. C. Leinweber and U. Tallarek, Nonequilibrium electrokinetic effects in beds of ion-permselective particles, Langmuir 20, 11637 (2004). - p..

[26] I. Rubinstein, B. Zaltzman, and I. Lerman, Electroconvective instability 
in concentration polarization and nonequilibrium electro-osmotic slip, Phys. Rev. E 72, 011505 (2005). - p.78, 80.

[27] I. I. Rubinstein and B. B. Zaltzman, Extended space charge in concentration polarization, Adv. Colloid Interface Sci. 159, 13 (2010). — p.72.

[28] V. S. Pham, Z. Li, K. M. Lim, J. K. White, and J. Han, Direct numerical simulation of electroconvective instability and hysteretic current-voltage response of a permselective membrane, Phys. Rev. E 86, 046310 (2012). - p.72.

[29] C. L. Druzgalski, M. B. Andersen, and A. Mani, Direct numerical simulation of electroconvective instability and hydrodynamic chaos near an ion-selective surface, Phys. of Fluids 25, 110804 (2013). — p.80.

[30] E. A. Demekhin, N. V. Nikitin, and V. S. Shelistov, Direct numerical simulation of electrokinetic instability and transition to chaotic motion, Phys. of Fluids 25, 122001 (2013). — p.72, 80.

[31] E. Hage and A. Tilgner, High Rayleigh number convection with double diffusive fingers, Phys. Fluids 22, 076603 (2010). — p.73.

[32] J. Raffel, M., Willert, C.E., Wereley, S.T., Kompenhans, Particle Image Velocimetry, A Practical Guide, 2nd ed. (Springer, 2007), p. 448 . — p.74.

[33] P. Tsai, A. M. Peters, C. Pirat, M. Wessling, R. G. H. Lammertink, and D. Lohse, Quantifying effective slip length over micropatterned hydrophobic surfaces, Phys. Fluids 21, 112002 (2009). — p.74.

[34] E. Karatay, A. S. Haase, C. W. Visser, C. Sun, D. Lohse, P. A. Tsai, and R. G. H. Lammertink, Control of slippage with tunable bubble mattresses, Proc. Natl. Acad. Sci. USA 110, 8422 (2013). — p.75.

[35] LaVision, Davis 8.2 Imaging software, 2014. — p.75.

[36] J. J. Krol, M. Wessling, and H. Strathmann, Chronopotentiometry and overlimiting ion transport through monopolar ion exchange membranes, J. Membr. Sci. 162, 155 (1999). — p.77, 78.

[37] V. V. Nikonenko, N. D. Pismenskaya, E. I. Belova, P. Sistat, P. Huguet, G. Pourcelly, and C. Larchet, Intensive current transfer in membrane systems: Modelling, mechanisms and application in electrodialysis, Advances in Colloid and Interface Science 160, 101 (2010). — p.77.

[38] D. R. Lide, Crc handbook of chemistry and physics, 76th ed. (CRC Press, 1996), pp. 5-90. - p.77.

[39] P. Długołecki, B. Anet, S. J. Metz, K. Nijmeijer, and M. Wessling, Transport limitations in ion exchange membranes at low salt concentrations, J. of Membr. Sci. 346, 163 (2010). — p.78.

[40] J. Balster, M. H. Yildirim, D. F. Stamatialis, R. Ibanez, R. G. H. Lammertink, V. Jordan, and M. Wessling, Morphology and microtopology of cation-exchange polymers and the origin of the overlimiting current., J Phys. 
Chem. B 111, 2152 (2007). — p.78.

[41] E. V. Dydek, B. Zaltzman, I. Rubinstein, D. S. Deng, A. Mani, and M. Z. Bazant, Overlimiting Current in a Microchannel, Phys. Rev. Lett. 107, 118301 (2011). - p.78.

[42] M. B. Andersen, M. van Soestbergen, A. Mani, H. Bruus, P. M. Biesheuvel, and M. Z. Bazant, Current-Induced Membrane Discharge, Phys. Rev. Lett. 109, 108301 (2012). - p.78.

[43] Supplementary material can be found online at http://prola.aps.org. p. 80 .

[44] C. L. Druzgalski, M. B. Andersen, and A. Mani, Direct numerical simulation of electroconvective instability and hydrodynamic chaos near an ion-selective surface, Physics of Fluids 25, 110804 (2013). - p.82. 


\section{CHAPTER 4}

\section{Effect of the gravitational orientation on the overlimiting current}

This chapter describes the influence of buoyancy on electroconvection at an ion exchange membrane in an aqueous electrolyte solution. Electrokinetic instabilities (EKI) and Rayleigh-Bernard convection (RB) are both known to mix the appearing concentration gradient layer and overcome the limiting current arising from diffusional limitations. The different physics, as well as the interplay between them, are investigated by resistance, flow and concentration characterization. In the buoyancy stable orientation, an EKI mixing layer, having a low concentration, grows till saturated size. In the buoyancy unstable orientation, $\mathrm{RB}$ occurs and dominates the advective transport due to the large system size. When $i<5 \cdot i_{\text {lim }} \mathrm{RB}$ mixes the system and EKI does not arise. If $i>5 \cdot i_{\text {lim }}$ EKI starts before RB and hastens the onset of RB. Upon onset of RB, EKI is suppressed while the overall resistance is still decreased. The onset times of EKI and $\mathrm{RB}$ are well predicted using a simple diffusion-migration model based on Fick's second law.

This chapter has been accepted for publication as:

Influence of Rayleigh-Bénard convection on electrokinetic instability in overlimiting current conditions, Joeri C. de Valença, Aziz Kurniawan, Jeffery A. Wood, R. Martijn Wagterveld, Rob G. H. Lammertink, Physical Review Fluids (2017) 


\subsection{Introduction}

For charge-selective interfaces such as ion-selective membranes or micro-nanochannel systems with Debye layer overlap, the existence of the so-called "limiting current", $i_{\lim }$, is a well-known theoretical and experimentally established phenomena (see $[1,2]$ for example). Currents above $i_{\text {lim }}$, called overlimiting current (OLC), have been observed experimentally and are related to additional transport mechanisms or water dissociation $[1,3,4]$. Electrically induced advection through electro-osmotic forces at the membrane interface in an extended space charge is a known mechanism behind OLC. The scientific path of theoretical prediction $[5,6]$ to experimental visualization [7-9] and more recent direct numerical simulations [10-12] has brought an understanding to the set of criteria that is needed to start this electrokinetic instability (EKI). Further research has been done on the 3D nature of EKI $[13,14]$ as well as the coupling with pressure driven flow $[14,15]$ and the modification of the membrane surface to reduce the resistance [16, 17]. Previously, we have investigated the coupling of electrokinetic instabilities to observed electrical signals, characterizing the transient dynamics of fluid vortices that arise [18].

Advective mixing can also be driven by buoyancy. When a less denser (ion depleted) fluid lies below a denser (ion enriched) fluid, gravitational forces can start mixing within the fluid. If the density gradient is parallel to the gravitational field the mixing only occurs above a critical gradient, identified with the Rayleigh number, called Rayleigh-Bernard (RB) convection [19-21]. At electrodes the morphology of the metal deposit is known to change due to RB convection [2224]. Buoyancy mixing of the boundary layer near a membrane is known to decrease the mass tranfer resistance [25-27]. Only recently a direct numerical simulation to study the coupling of RB and EKI near a membranes has been done [28]. The estimated currents were greatly influenced by the appearance of $\mathrm{RB}$ convection. No universal relation could be obtained for the current, as the $\mathrm{RB}$ and EKI were predicted to be coupled in highly nonlinear fashion.

This chapter reports the results of the experimental investigations on the influence of Rayleigh-Bernard convection, the electrokinetic instability, and coupling between these phenomena in a cation exchange membrane system. The changing concentration profile, flow and electrical response of the setup for different orientations of electric field with respect to the gravitational field is experimentally quantified. This is accomplished using fluorescence lifetime imaging microscopy (FLIM), particle image velocimetry (PIV) and potentiostatic measurements From the dynamic measurements the convection onset times are compared to a simple model based on Fick's laws of diffusion. 


\subsection{Material and methods}

\subsubsection{Electrochemical measurements (Chronopotentiometry)}

Figure 4.1 shows the experimental setup, comprising of a cation exchange membrane, (Neosepta CMX), with surface area $A_{\text {mem }}=3 \times 4.5 \mathrm{~mm}^{2}$, and thickness of $170 \mu \mathrm{m})$, placed between two reservoirs filled with a $10 \mathrm{mM} \mathrm{CuSO}_{4}$ electrolyte solution, both closed with copper electrodes. Chronopotentiometric measurements are performed by forcing a constant DC electric current, $I$, through the setup (Autolab PGSTAT 30). The anode, where copper oxidizes, serves as a $\mathrm{Cu}^{2+}$ cation source, and the cathode, where copper reduces, acts as a $\mathrm{Cu}^{2+}$ cation sink. The time-dependent voltage difference, $\Delta V(t)$, was measured between the anode and a copper wire placed on the other side of the membrane. The current density was set between $i=4.4-44.4 \mathrm{~A} / \mathrm{m}^{2}$.

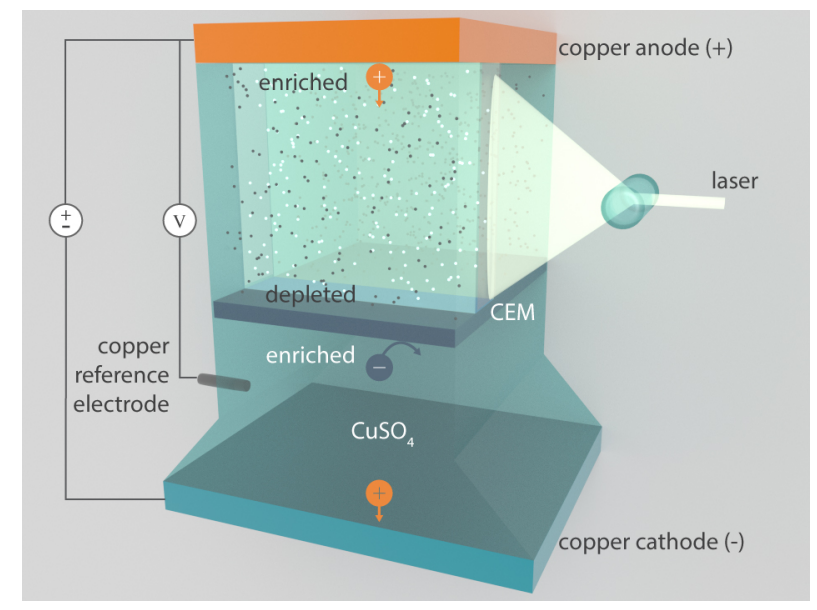

Figure 4.1: Experimental setup in co-orientation, the buoyancy unstable situation, where the electric and gravitational field point downwards, noted as $\mathrm{g} \downarrow \mathrm{E} \downarrow$. The voltage drop, $\Delta V$, under a constant applied current, $I$, is measured simultaneously with the local hydrodynamics of the ionic solution inside the anode compartment.

The dynamics is investigated for two different orientations: 1. The membrane is placed horizontal with anode compartment above it, figure 4.1. This called the co-orientation, since the electric field direction (anode to cathode) is aligned with the gravitational field (buoyancy unstable). 2. The cell is rotated $180^{\circ}$ which we call the counter-orientation (buoyancy stable). 


\subsubsection{Flow measurements (PIV)}

Flow dynamics in the anode compartment are measured by seeding the solution with $0.1 \mathrm{wt} \% 2 \mu \mathrm{m}$ red polystyrene tracer particles (Microparticles $\mathrm{GmbH}$, with a density of $1.05 \mathrm{~g} / \mathrm{cm}^{3}$ ). The particles are illuminated with a thin laser sheet (Firefly, Oxford lasers), see figure 1, and the reflected light is captured through a long distance magnifying lens (Navitar) on a camera at 10 or 32 frames per second. From the recorded particle displacement we determine the vector field using particle image velocimetry (PIV) analysis (DaVis), figure 4.3-middle.

Recording of images Depending on the particle velocity and magnification an appropriate recording frequency is chosen. The movie and PIV analysis in of the mixing layer in the counter-orientation at $29.6 \mathrm{~A} / \mathrm{m}^{2}$ was done with $\Delta t=$ $0.03 \mathrm{~s}$. All other movies and PIV analysis is done with $\Delta t=0.1 \mathrm{~s}$. The typical magnification of the magnifying lenses attached to the camera is $M=2.5$ (with the resolution of 1 pixel $=2 \mu \mathrm{m}$ ). The entire cell, $2 \times 3 \mathrm{~mm}$, is in view.

Masking A geometrical mask covers the membrane, anode and side wall so no vector field is calculated in that region.

Multigrid vector calculation To calculate the flow field, a multigrid cross correlation method with decreasing window size is used. First a $98 \times 98$ pixel interrogation window is used to determine a reference vector field. This field is then used to calculate a window shift of the next correlation. This shift ensures the same particles are correlated with each other. To get a higher resolution the second calculation is done with windows of $48 \times 48 \mathrm{pixel}^{2}$.

Vector postprocessing To eliminate erroneous vector a LaVision vector post-processing algorithm is used. The same median bases outlier detection algoritms as described in Chapter 3 is used.

Temporal averaging The dynamics are relatively slow, so for five consecutive vector fields the orthogonal components are averaged separately (sliding average) to reduce the influence of erroneous vectors further. Final vector fields thus represent the average motion during $\Delta t=0.5 \mathrm{~s}$, or $\Delta t=0.16 \mathrm{~s}$ in case of $29.6 \mathrm{~A} / \mathrm{m}^{2}$ in the counter-orientation.

Determining the vortex size and speed In the co-orientation the motion occurs in the full cell and root mean square of all vectors of the final vector field is taken as the mean velocity of the mixing layer. In the counter-orientation first the mixing layer thickness $\left(L_{m i x}\right.$ or vortex size) needs to be determined from the final vector field before the vortex speed (root mean square velocity in the vortex area) can be calculated. The vortex boundary is characterized by the sharp decay of the vertical velocity component, $v_{y}$, away from the membrane. From a horizontal row (40 vectors) the average value is taken. These average values are interpolated with a cubic spline to get a smooth function representing 
the change in vertical velocity. The vortex region is quantified by the distance between the membrane position (determined with ImageJ) and position where the vertical velocity drops below a fixed threshold value. The threshold value is taken as $10 \%$ of the maximum value inside the vortex region. The absolute value of the vectors in this vortex region is averaged to obtain the vortex speed. A similar technique was used in our previous paper [18].

\subsubsection{Concentration measurements (FLIM)}

The concentration polarization is determined using a fluorescence lifetime image microscopy (FLIM) system (LaFLIM, Lambert instruments, Groningen, The Netherlands). The fluorescence decay of Alexa Fluor 488 dye depends on the $\mathrm{CuSO}_{4}$ concentration in the range between 1 and $100 \mathrm{mM}$, as seen in the calibration curve and error estimation, see figure 4.2. A modulated blue LED light is sent through a $5 \mathrm{x}$ magnifying objective (Zeiss) and the fluorescence is captured on a $696 \times 520$ pixels CCD camera. Each pixel represents an area of $4.17 \times 4.17 \mu \mathrm{m}^{2}$. To have sufficient light intensity each image was made with a shuttertime of $\Delta t=300 \mathrm{~ms}$. From 12 phase images the shift of fluorescent lifetime of each pixel is determined compared to a reference images. The reference is demineralized water (milliQ) with $2.5 \mu \mathrm{M}$ Alexa Fluor ${ }^{\circledR} 488$ dye. The lifetime of that sample is set to $4.1 \mathrm{~ns}$, according to literature $[29,30]$.
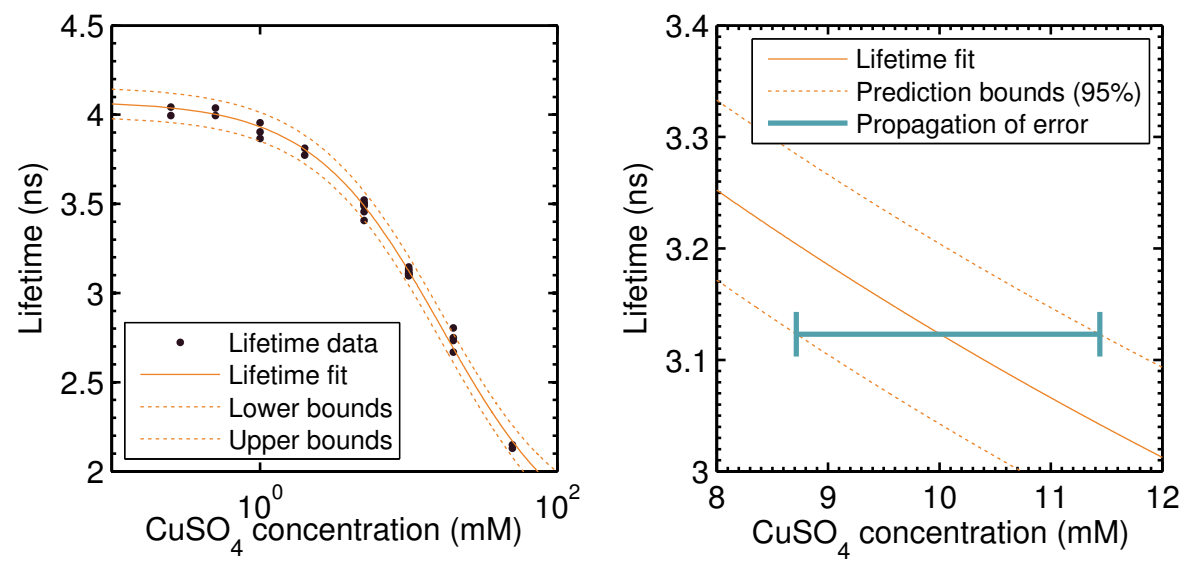

Figure 4.2: Left: FLIM calibration curve on semi-log scale. Right: From the prediction bounds, an estimate on the inverse-error of determining concentration from lifetime can be determined

The fluorescence decay of Alexa Fluor 488 dye is concentration dependent 
in the range between 1 and $100 \mathrm{mM}$, see figure 4.2a. A modified Stern-Volmer equation is used to fit the data [31]:

$$
\tau=A\left(1-\frac{1}{\frac{1}{f K x}+\frac{1}{f}}\right)
$$

Which fitted best with $A=4.0761 \pm 0.0263, K=0.0598 \pm 0.0058$ and $f=$ $0.6243 \pm 0.0250$. $A$ corresponds (within error) to the expected lifetime of Alexa dye in water $(\sim 4.1 \mathrm{~ns})$ [30]. From the confidence intervals the prediction bound of $95 \%$ is determined. Once the lifetime is measured the errorbar in concentration is derived via this prediction bound, see figure $4.2 \mathrm{~b}$.

\subsubsection{Numerical model of concentration dynamics}

Finally, the degree of concentration polarization in the anode compartment is predicted using a transient 1D formulation of Fick's second law. This follows from combining the Nernst-Planck equation with the continuity relation and electroneutrality assumption [32]. The membrane and anode are assumed to be ideally cation selective. The initial condition is a homogeneous concentration and the boundary conditions are set by the applied constant current. At the anode there is a constant inflow of cations and at the membrane a constant outflow. The development of the concentration gradients are solved via a PDE solver (MATLAB), an analytical solution can also be found in [32]. This allows for prediction of concentration gradient and onset times of EKI and RB in the cell. The equations read:

$$
\frac{\partial c(x, t)}{\partial t}=D \frac{\partial^{2} c(x, t)}{\partial x^{2}}
$$

with the initial and boundary conditions:

$$
\begin{aligned}
c(x, 0) & =c_{0} \\
\frac{\partial c(0, t)}{\partial x} & =-\frac{i}{z F D}\left(\overline{t_{+}}-t_{+}\right) \\
\frac{\partial c(L, t)}{\partial x} & =-\frac{i}{z F D}\left(t_{+}-\hat{t_{+}}\right)
\end{aligned}
$$

where $c(x, t)$ is the concentration, $\mathrm{z}$ is the valency of the (symmetric) electrolyte $(z=2), F$ is Faraday constant $\left(F=9.65 \cdot 10^{4} \mathrm{C} / \mathrm{mol}\right)$ and $\mathrm{D}$ is the diffusion coefficient $\left(D_{\mathrm{CuSO} 4}=0.855 \cdot 10^{9} \mathrm{~m}^{2} / \mathrm{s}[33]\right)$. We assume an ideal membrane and electrode that only allow cations to move through with transport number, 
$\overline{t_{+}}=\hat{t_{+}}=1$. The bulk transport number reflects the mobility of $\mathrm{Cu}^{2+}$ compared to $\mathrm{SO}_{4}{ }^{2-}, t_{+}=D_{+} /\left(D_{+}+D_{-}\right)=0.4$ [34]. This is the part of the current that is carried by cations in the bulk. Due to the slab symmetry the concentration only changes perpendicular to the membrane, where $x=0$ at the membrane and $x=L$ at the anode.

The steady state condition of the model is a constant gradient throughout the whole cell. The gradient is linear proportional to the applied current. The limiting current arises if $c_{\text {mem }}=0$. The value of the limiting current density can also be predicted with Peers equation [34]:

$$
i_{\lim }=\frac{z F D}{\left(\overline{t_{+}}-t_{+}\right)} \frac{c_{\delta}}{\delta}
$$

where $\delta$ represents the length of the stagnant diffusion layer and $c_{\delta}$ the concentration at the end of this layer. For $\delta=2 \mathrm{~mm}$ and $c_{\delta}=2 \cdot c_{0}=20 \mathrm{mM}$ and ideal boundaries a limiting current density of $2.7 \mathrm{~A} / \mathrm{m}^{2}$ is obtained.

\subsection{Results and discussion}

The result and discussion section is separated in three subsections. Firstly, the difference in steady state dynamics is discussed. Only in the co-orientation Rayleigh-Bénard convection (RB) occurs. It reduces the ion concentration polarization (ICP) and avoids or diminishes electroconvection that occurs via the electrokinetic instability (EKI), thereby decreasing the electrolyte resistance compared to the co-orientation. Secondly, the transition time of both types of convection is determined and shows the agreement between experiment (electrical and flow) and the theory that predicts when EKI can occur. Thirdly, concentration measurements verify the theory further and a numerical $1 \mathrm{D}$ framework is constructed that predicts the onset of RB. Different current regimes are identified in which the interplay between the two types of convection is fundamentally different.

\subsubsection{Steady state}

In counter-orientation, currents above $i_{\text {lim }}$ result in EKI and no RB convection was observed as expected, since the ion depleted fluid lies above the ion enriched fluid $[19,35]$. In figure 4.3-top the typical flow field next to the membrane is shown while applying $29.6 \mathrm{~A} / \mathrm{m}^{2} \approx 11 \cdot i_{\text {lim }}$ when the mixing layer is fully developed $(t=300 \mathrm{~s})$. The top half of the cell shows electroconvective mixing, while the bottom half is relatively stagnant, although clearly showing secondary vortex motion [36]. The boundary of the mixing layer is taken as the distance 
from the membranes where the mean rms velocity is $10 \%$ of the maximum, see figure 4.3-right. In figure 4.4 the the mean rms velocity within the mixing layer and the applied voltage difference are shown with respect to time, which reflect the strong chaotic fluctuations in the velocity and applied voltage. The fluctuations are not a result of measurement error, since the temporal resolution of both electrical and flow measurements was sufficient. Careful analysis did not reveal a correlation between the signals. It has to be noted that the voltage is applied over the full volume, while the velocity field is extracted from a cross-section. Combining the average additional voltage, $\Delta V_{\text {olc }}=3.4 \mathrm{~V}$ with the average mixing size, $L_{\text {mix }} \approx 1 \mathrm{~mm}$, estimates the conductivity of the mixing layer, $\sigma=L_{\text {mix }} i / \Delta V_{\text {olc }} \approx 9 \cdot 10^{1} \mu \mathrm{S} / \mathrm{cm}$. The conductivity of the mixing layer has a similar value as found in smaller less chaotic vortices as observed in the previous Chapter.
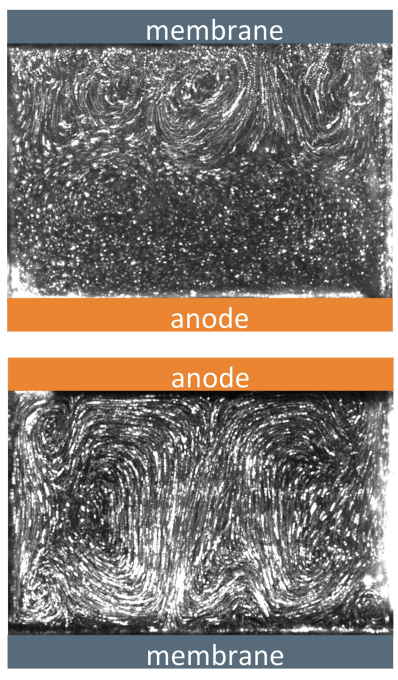
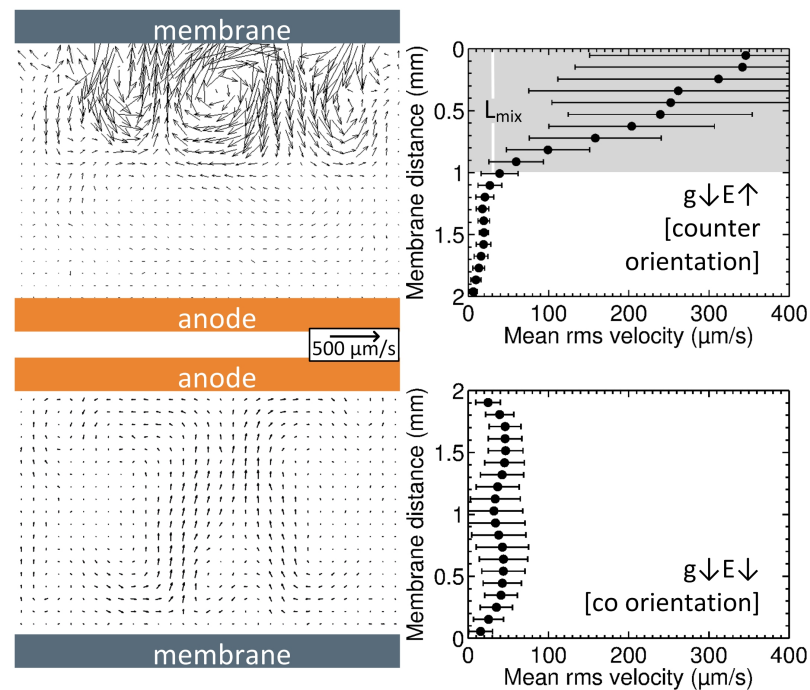

Figure 4.3: Left column: Particle pathlines over 5 s. Middle column: Aaverage velocity field over $0.1 \mathrm{~s}$. Right column: Distribution of the mean rms velocity of a row of 31 vectors with standard deviation as a function of the height in the reservoir. Data from $29.6 \mathrm{~A} / \mathrm{m}^{2} \approx 11 \cdot i_{\mathrm{lim}}, 10 \mathrm{mM} \mathrm{CuSO}_{4}, \mathrm{t}=300 \mathrm{~s}$ in counter orientation (top row) and co orientation (bottom row).

When the system is rotated 180 degrees (to co-orientation, i.e. buoyancy unstable) substantially different behaviour is observed. Flow occurs in the whole cell, see figure 4.3-bottom. Fluid motion mixes the high concentration at the anode with the low concentration at the membrane. The ICP diminishes and the 
resistance decreases towards the initial ohmic resistance, as shown in figure 4.4top. Gravitational or Rayleigh-Bérnard convection occurs if the destabilizing buoyancy forces overcome stabilizing viscous forces [21,37,38]. This ratio is expressed by the non-dimensional Rayleigh number:

$$
R a=\frac{M \Delta c g L^{3}}{\mu D}
$$

where $M=0.1567 \mathrm{~kg} / \mathrm{mol}$ is the molar mass of the $\mathrm{CuSO}_{4}, g=9.81 \mathrm{~m} / \mathrm{s}^{2}$ is the gravitational constant, $\mu=1.002 \cdot 10^{-9} \mathrm{~kg} / \mathrm{m} / \mathrm{s}$ is the dynamic viscosity of water, and $D=D_{\mathrm{CuSO}_{4}}=8.55 \cdot 10^{-10} \mathrm{~m}^{2} / \mathrm{s}$ is the diffusion coefficient of copper sulphate.

The RB instability is found to occur if $R a>R a_{\mathrm{c}}$. The critical Rayleigh number depends on the system parameters but is on the order of $R a_{\mathrm{c}}=1000$, as in [19], which concerned a similar electrolyte system with flat sheet copper electrodes in $\mathrm{CuSO}_{4}$ solution. This value is also in agreement to other numerical work on the effect of nonlinear gradients between two rigid boundaries (no-slip) with constant flux and constant concentration boundary values [39]. For the limiting current condition $(L=2 \mathrm{~mm}, c=20 \mathrm{mM})$ the Rayleigh number is $R a=2.9 \cdot 10^{5}$. The experimental observation of RB convection is therefore expected above the limiting current and agrees with other work [26, 38]. Rayleigh-Bénard convection is also observed at underlimiting currents. RB flow brings denser (ion rich) fluid down to the membrane, while less dense (ion depleted) fluid moves away. This balances the anion migration away from the membrane and diminishes the ion depletion.

At $i=8 \cdot i_{\mathrm{lim}}$ and higher a small layer with EKI is present at the membrane while RB convection dominates the rest of the cell. The EKI layer size $O(100 \mu \mathrm{m})$ is larger at higher currents. The enhanced ion transport caused by RB is not sufficient to sustain the high current demand and avoid depletion of the interface. These observations confirm the numerical predictions of Karatay [28]. It could not be determined in our case if the EKI vortices stayed counter-rotating [8] or the RB flow caused co-rotating [40] or even helical [14] vortices.

\subsubsection{Transition time}

The onset of the instabilities gives an understanding in the coupling of both types of convection. RB mixing starts when the concentration gradient becomes buoyancy unstable and mixes the whole volume [20,21]. EKI mixing starts at the membrane and occurs after the membrane surface becomes depleted and an overvoltage is applied $[5,6,10]$. The time for $c_{\mathrm{mem}}=0$ can be determined 

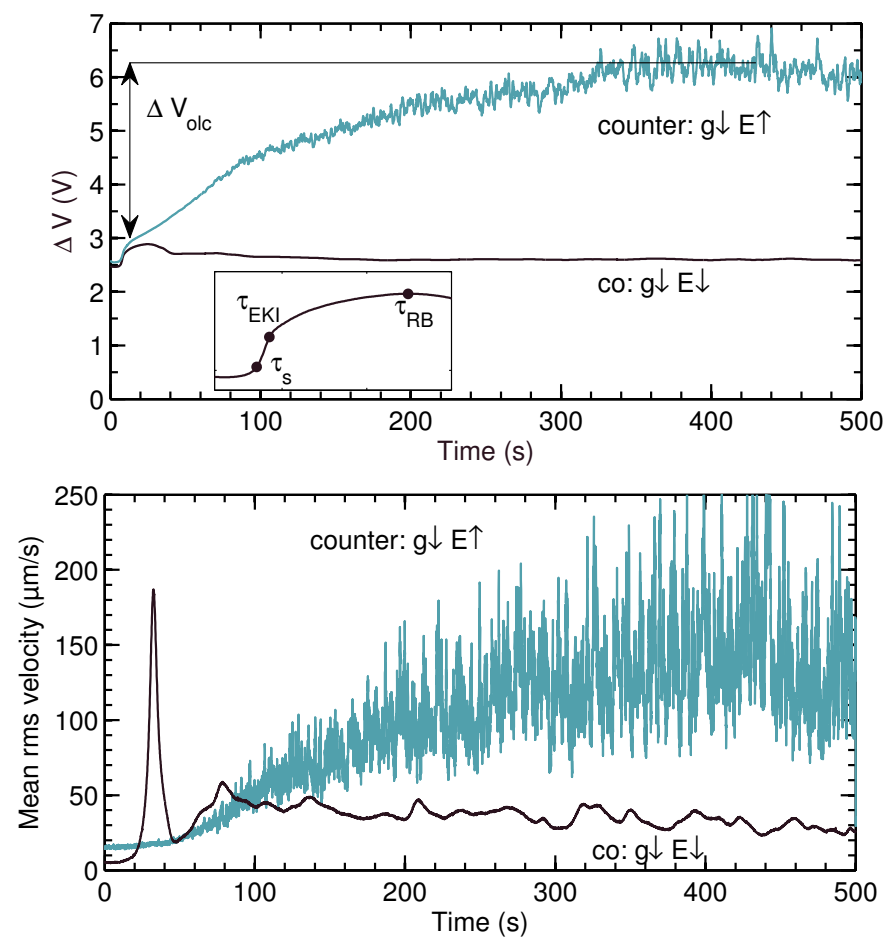

Figure 4.4: The voltage drop, $\Delta V$, (top) and mean rms velocity of the PIV vectors in the full cell (bottom) versus time for $29.6 \mathrm{~A} / \mathrm{m}^{2}$ in $10 \mathrm{mM} \mathrm{CuSO}_{4}$ in the co and counter orientation. The inset in the top figure shows the electrical transition times in the co-orientation.

from the diffusion-migration model. For an infinitely large system, $L \rightarrow \infty$, an analytical solution can be derived known as Sand's transition time [41]:

$$
\tau_{s}=\frac{\pi D}{4}\left(\frac{c_{0} z F}{\overline{t_{+}}-t_{+}}\right)^{2} \frac{1}{i^{2}}
$$

For $c(L=1 \mathrm{~mm})=c_{0}$ this agrees with Sand's equation when the diffusion layer is smaller than $L$. It was verified that at the lowest current $\left(4.4 \mathrm{~A} / \mathrm{m}^{2}=1.6 \cdot i_{\text {lim }}\right)$ the numerical depletion time is similar to $\tau_{\mathrm{s}}$. An analytical function for the transition time that is also valid at even lower currents can be found in [42].

From the chronopotentiometric measurements response, the time for ion depletion at the membrane interface is extracted, since it causes a jump in resistance $[18,43]$. The inset in figure 4.4-top shows the electrical signature of the transitions. The depletion time is usually taken as the base of voltage jump [43], or 
the point with maximal gradient [44]. To extract the value of the voltage jump in a consistent way the depletion time, $\tau_{\mathrm{s}}$, is taken as the first point where the rate of change of the gradient is maximum $\left(\partial_{t}^{3} V(t)=0\right)$. The end of the voltage jump and start of EKI, $\tau_{\mathrm{EKI}}$, is taken as the second point where $\partial_{t}^{3} V(t)=0$. The electrical RB transition time, $\tau_{\mathrm{RB}}$, is taken as the moment the RB mixing decreases ICP and overall resistance $\left(\partial_{t} V(t)=0\right)$.

From the simultaneous flow measurements the start of EKI, $\tau_{\text {EKI }}$, is taken when the first particle movement next to the membrane appears. The flow signature of $\mathrm{RB}$ convection, $\tau_{\mathrm{RB}}$, is taken as the instant the full cell velocity suddenly jumps up. Figure 4.5-left shows the root mean square (rms) velocity of the vector field of the whole membrane compartment as measured with PIV. After the concentration gradient becomes unstable RB convection starts and the rms velocity increases steeply. The base of the velocity peak is taken as the moment the increase in speed is highest $\left(\partial_{t}^{3} v_{\mathrm{rms}}(t)=0\right)$. This is the inflection point of the first derivative and local maximum of the second derivative.
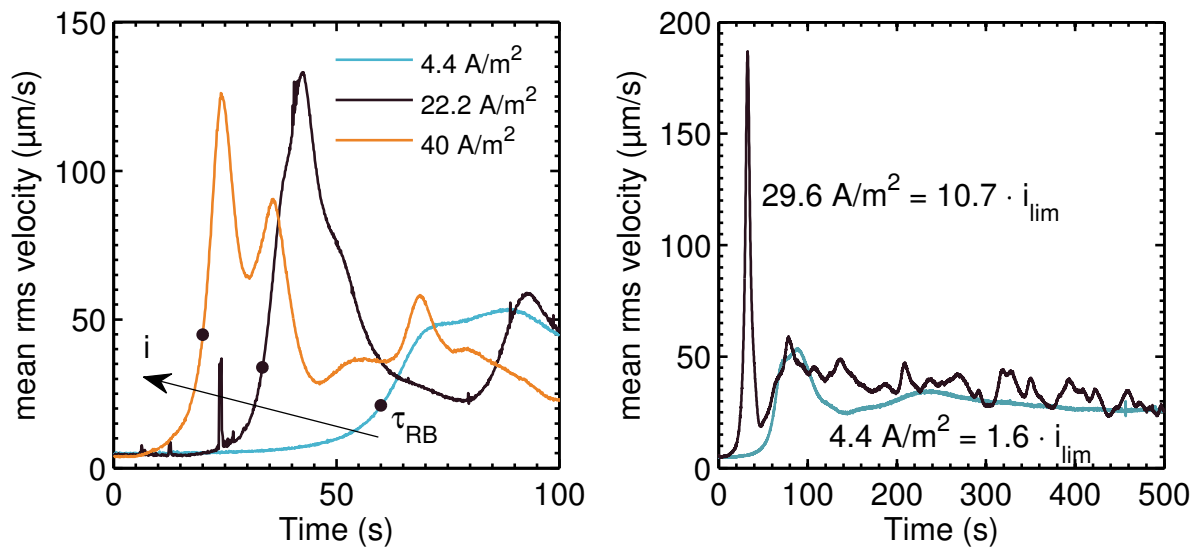

Figure 4.5: Left: The growth of the mean rms velocity occurs earlier $\left(\tau_{\mathrm{RB}}\right)$ and faster at higher currents. The black dot $(\bullet)$ represents the determined base of the peak. Right: The peak velocity increases with increased current, but final average velocity seems independent on current. At higher currents there is more fluctuation.

The velocity fluctuation at the beginning at higher currents, $i>6.4 \cdot i_{\text {lim }}=$ $18 \mathrm{~A} / \mathrm{m}^{2}$, is caused by mixing via EKI at the membrane. Although the velocity inside the EKI mixing layer is high, the full cell rms velocity is low, because the layer is initially small. A higher current gives a higher peak velocity and an earlier onset. The gradients build up faster and the concentration difference between the membrane and the anode becomes higher. After the mixing starts the gradients 
are reduced and the velocity drops. Even after $500 \mathrm{~s}$, figure 4.5-right, the velocity still decreases.

\subsubsection{Concentration profile development}

In the counter-orientation the concentration profile development was determined using fluorescence lifetime imaging microscopy (FLIM). In figure 4.6 two images of the concentration distribution in the anode compartment as derived from FLIM are displayed. They show the concentration polarization at $t=45-60 \mathrm{~s}$ and $t=825-840 \mathrm{~s}$ while applying $i=3.3 \cdot i_{\text {lim }}=8.9 \mathrm{~A} / \mathrm{m}^{2}$ inside a $10 \mathrm{mM}$ $\mathrm{CuSO}_{4}$ solution with $2.5 \mu \mathrm{M}$ Alexa dye. From these images a representative 1D concentration profile is calculated as shown in figure 4.7-top-left. Each data point is obtained from averaging the lifetime over 100 pixels (along membrane in the center) by 20 pixels (normal to membrane).
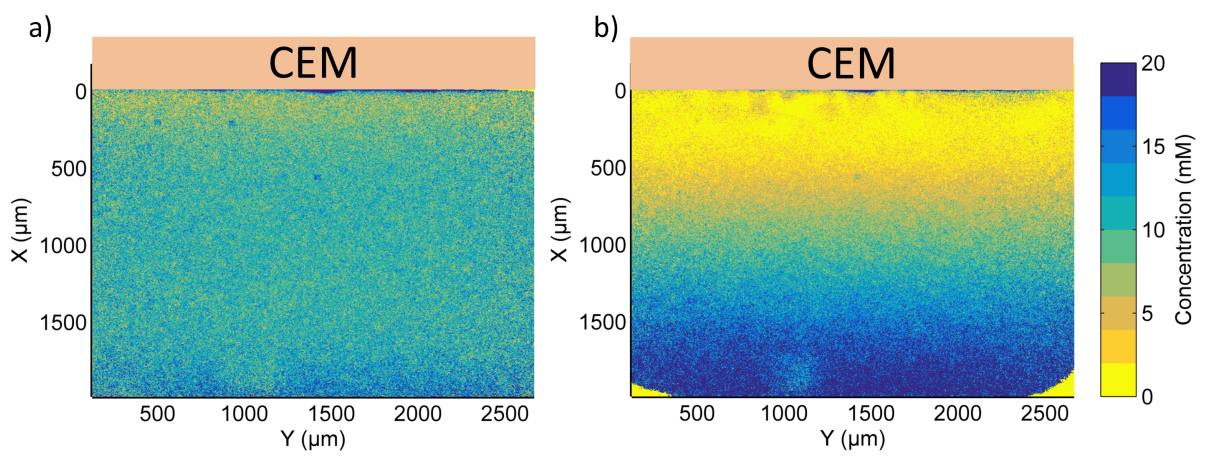

Figure 4.6: Images of the FLIM lifetime of each pixel colour coded from blue $2.5 \mathrm{~ns}$ to red $4.1 \mathrm{~ns}$. The cation exchange membrane is at the top and $i=3.3 \cdot i_{\text {lim }}=8.9 \mathrm{~A} / \mathrm{m}^{2}$. Left: t=45-60 s. Right: $825-840 \mathrm{~s}$

With the numerical model the concentration profile between the membrane and anode is predicted, which is shown in figure 4.7-top-left. The experimental system displays a diffusion layer and a mixing layer with a concentration, $c_{\text {mix }}=0.1 \cdot c_{0}=1 \pm 0.2 \mathrm{mM}$. This is in line with a previous postulate that the overlimiting resistance, $R_{\text {olc }}=\Delta V_{\text {olc }} /\left(i A_{\text {mem }}\right)$ is mainly caused by a layer with low concentration (chapter $3,[18]$ ).

In co-orientation, the model can be used to predict when the boundary layer becomes unstable. The flow observations show the start of half cell RB vortices, most pronounced at the anode, with much faster full cell motion starting quickly after. To estimate when the half cell becomes unstable, $R a>R a_{\mathrm{c}}$, a characteristic length is chosen from the nonlinear concentration gradient. For this, the 

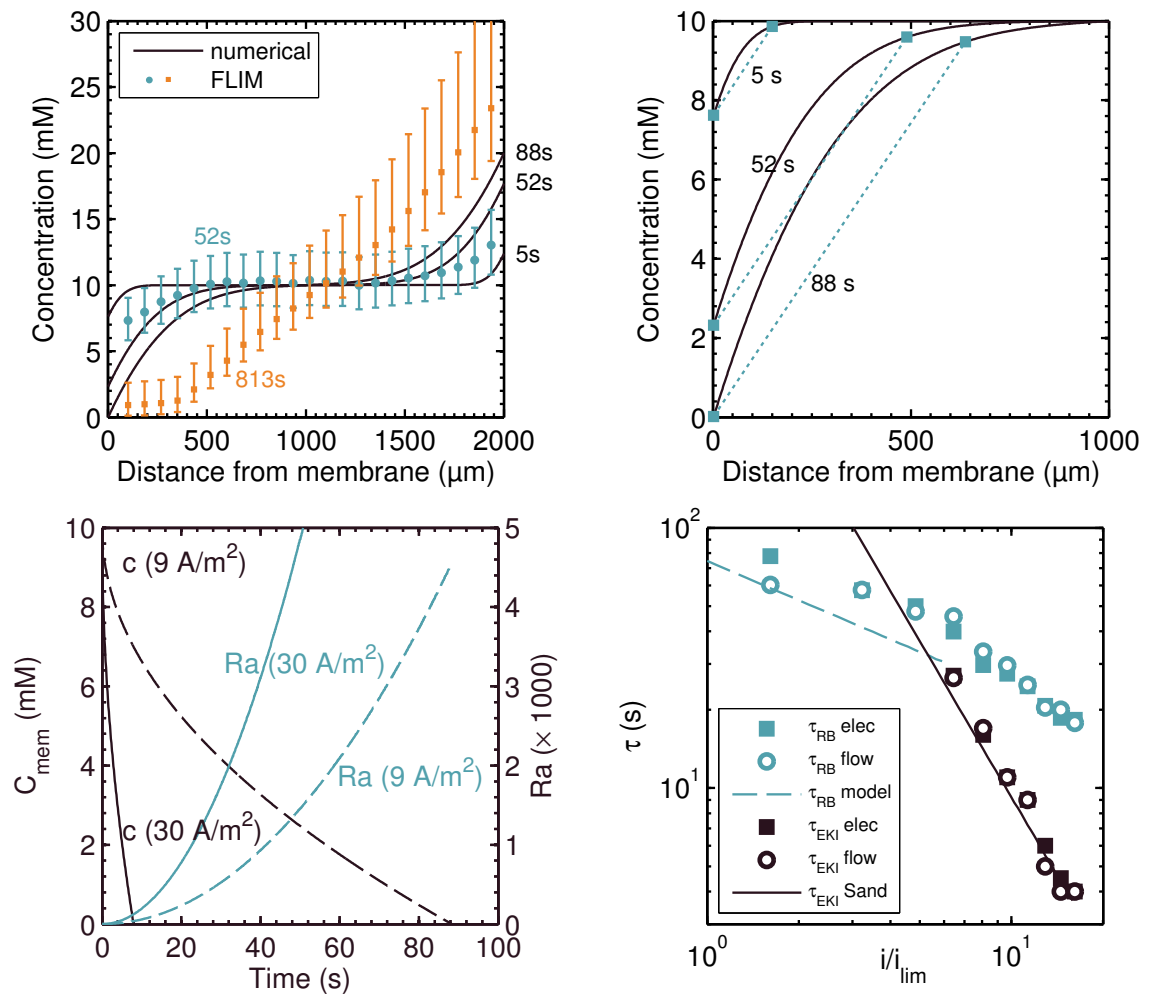

Figure 4.7: Top-left: shows the numerical (black line) and experimental concentration profile (FLIM). At overlimiting current ( $812 \mathrm{~s}$ ) the mixing layer has a low concentration. Top-right: shows the numerical estimated concentration profile (black solid line) for $i=8.9 \mathrm{~A} / \mathrm{m}^{2}=3.3 \cdot i_{\text {lim }}$ at different times. The blue dotted line shows the characteristic length and concentration difference that are used to determine $R a . \mathrm{t}=5$ s gives $R a=14$. $\mathrm{t}=52 \mathrm{~s}$ gives $R a=1.5 \cdot 10^{3}$. $\mathrm{t}=5 \mathrm{~s}$ gives $R a=4.5 \cdot 10^{3}$. Bottom-left: shows the numerical prediction of the membrane concentration and the Rayleigh number of the boundary layer in time. Bottom-right: shows the experimental transition times (points) vs the applied current scaled to the limiting current in log-log scale. The lines show the numerical prediction of onset of RB convection and EKI mixing.

distance between the membrane or anode and the point where the concentration gradient is $10 \%$ of the maximum value is taken. In figure 4.7 -top-right this characteristic length, $L$, together with the concentration difference, $\Delta c$ is shown. With these values the Rayleigh number of the boundary layer is calculated, which is plotted against time in figure 4.7-bottom-left. From this line the time when $R a>1000$ is determined, which is taken as the numerical RB transition time, 
$\tau_{\mathrm{RB}} \cdot$

An overview of all co-orientation transition times is given in figure 4.7-bottomright. The electrical and flow EKI transition times, $\tau_{\mathrm{EKI}}$, are similar and match well with the theoretical depletion time. The EKI onset thus follows a -2 power law with the current as seen in the Sand's equation and found in many other experiments $[18,43]$. The transition time for the RB convection decays slower with increased current. This leads to two regimes, one where RB convection occurs before EKI and one where EKI starts first. The presented numerical model predicts a decay of power $\sim-0.5$, which comes close to the actual data. If EKI starts, this hastens the onset of RB convection and the data deviates from this -0.5 power prediction.

Once a single boundary layer becomes unstable, due to RB convection or EKI mixing, the whole system becomes unstable. The sudden increase in characteristic length scale $(L)$ and concentration difference $(\Delta c)$ causes full cell mixing which reduces the ICP and resistance. When the driving force diminishes, the mean rms velocity decreases with a damped oscillation to a flow with one or two full cell vortices.

\subsection{Conclusion}

In summary, this work experimentally characterizes the influence of buoyancy effects (RB, Rayleigh-Bernard) and the electrokinetic instability (EKI) in overlimiting current conditions. In counter-orientation, which is buoyancy stable, an EKI mixing layer and an almost stagnant diffusion layer are identified. The concentration and velocity measurements indicate that the high OLC resistance is a result of the low concentration in the mixing layer. In co-orientation, which is buoyancy unstable, both RB convection and EKI mixing can occur. The presented model and experimental data on the onset of both types of convection are in agreement. For $i<5 \cdot i_{\text {lim }}$ the RB onset time is shorter compared to the Sand transition time and RB convection prevents ion depletion at the membrane. EKI is found to start before RB if $i>5 \cdot i_{\text {lim }}$ and that this accelerates the onset of RB due to the disturbance of the depletion layer by EKI. At currents above $i>7 \cdot i_{\lim }$ EKI stays present at the membrane interface, indicating that the $\mathrm{RB}$ convection cannot avoid depletion. The mixing of the full cell decreases the resistance to near initial values. This mixing causes enhanced ion transport than diffusion allows for at limiting current conditions. 


\section{A Supplemental movies}

Four supporting movies are prepared. The first three supporting movies show the simultaneous flow and electrical measurements presented in figure 4.4. The current was kept constant at $29.6 \mathrm{~A} / \mathrm{m}^{2}\left(i / i_{\text {lim }} \sim 11\right)$.

Movie 1 shows the dynamics in the counter-orientation for $500 \mathrm{~s}$. Each frame is made of an overlay of 50 images (Image J, Z project, max intensity) representing $1.7 \mathrm{~s}$ of motion (30 fps). The corresponding voltage drop and the mean rms velocity in the full cell is shown below. Around $t=10 \mathrm{~s}$ EKI mixing starts and grows till saturation. No RB occurs.

Movie 2 shows the dynamics in the co-orientation for $500 \mathrm{~s}$. Each frame is made of an overlay of 50 images (Image J, Z project, max intensity) representing $5 \mathrm{~s}$ of motion (10 fps). The corresponding voltage drop and the mean rms velocity in the full cell is shown below. Around $t=10 \mathrm{~s}$ EKI mixing starts and grows till RB occurs.

Movie 3 displays the first $75 \mathrm{~s}$ in the co-orientation. The flow fields (every $1 \mathrm{~s})$ are obtained with PIV analysis. The vector length represents the velocity size and direction. The background color represents the calculated vorticity. Together with the time evolving flow velocity vectors, the voltage response and the calculated mean rms velocity in the full cell are shown.

Movie 4 supports the data presented in figure $4 \mathrm{a}$. The concentration polarization after applying $9 \mathrm{~A} / \mathrm{m}^{2}\left(i / i_{\lim } \sim 3\right)$. is visualized with fluorescence lifetime imaging microscopy (FLIM). Each frame shows the average of the concentration in $15 \mathrm{~s}$. After $\approx 100 \mathrm{~s}$ EKI mixing starts and an mixing layer with low concentration appears. At $1000 \mathrm{~s}$ the electric forcing stops as well as the EKI mixing. The concentration gradient diminishes in time due to diffusion. 



\section{Bibliography}

[1] V. V. Nikonenko, A. V. Kovalenko, M. K. Urtenov, N. D. Pismenskaya, J. Han, P. Sistat, and G. Pourcelly, Desalination at overlimiting currents: State-of-the-art and perspectives, Desalination 342, 85 (2014). — p.94.

[2] H. C. Chang, E. A. Demekhin, and V. S. Shelistov, Competition between Dukhin's and Rubinstein's electrokinetic modes, Physical Review E 86, (2012). - p.94.

[3] T. A. Zangle, A. Mani, and J. G. Santiago, On the propagation of concentration polarization from microchannel- nanochannel interfaces Part II: numerical and experimental study, Langmuir 25, 3909 (2009). — p.94.

[4] J. J. Krol, M. Wessling, and H. Strathmann, Concentration polarization with monopolar ion exchange membranes: current-voltage curves and water dissociation, Journal of Membrane Science 162, 145 (1999). — p.94.

[5] S. S. Dukhin, Electrokinetic phenomena of the second kind and their applications, Advances in Colloid and Interface Science 35, 173 (1991). — p.94, 101.

[6] I. Rubinstein and B. Zaltzman, Electro-osmotically induced convection at a permselective membrane, Physical Review E 62, 2238 (2000). — p.94, 101.

[7] S. Kim, Y.-C. Wang, J. Lee, H. Jang, and J. Han, Concentration Polarization and Nonlinear Electrokinetic Flow near a Nanofluidic Channel, Physical Review Letters 99, 044501 (2007). - p.94.

[8] S. Rubinstein, G. Manukyan, A. Staicu, I. Rubinstein, B. Zaltzman, R. Lammertink, F. Mugele, and M. Wessling, Direct Observation of a Nonequilibrium Electro-Osmotic Instability, Physical Review Letters 101, (2008). - p.101.

[9] G. Yossifon and H.-C. Chang, Selection of Nonequilibrium Overlimiting Currents: Universal Depletion Layer Formation Dynamics and Vortex Instability, Physical Review Letters 101, 254501 (2008). — p.94.

[10] C. L. Druzgalski, M. B. Andersen, and a. Mani, Direct numerical simulation of electroconvective instability and hydrodynamic chaos near an ion-selective surface, Physics of Fluids 25, 110804 (2013). - p.94, 101.

[11] E. a. Demekhin, N. V. Nikitin, and V. S. Shelistov, Direct numerical simulation of electrokinetic instability and transition to chaotic motion, Physics 
of Fluids 25, 122001 (2013). - p..

[12] V. S. Pham, Z. Li, K. M. Lim, J. K. White, and J. Han, Direct numerical simulation of electroconvective instability and hysteretic current-voltage response of a permselective membrane, Physical Review E 86, (2012). p.94.

[13] E. a. Demekhin, N. V. Nikitin, and V. S. Shelistov, Three-dimensional coherent structures of electrokinetic instability, Physical Review E 90, 013031 (2014). - p.94.

[14] S. V. Pham, H. Kwon, B. Kim, J. K. White, G. Lim, and J. Han, Helical vortex formation in three-dimensional electrochemical systems with ion-selective membranes, Physical Review E 93, 033114 (2016). — p.94, 101.

[15] R. Kwak, V. S. Pham, K. M. Lim, and J. Han, Shear Flow of an Electrically Charged Fluid by Ion Concentration Polarization: Scaling Laws for Electroconvective Vortices, Physical Review Letters 110, 114501 (2013). — p.94.

[16] J. Balster, M. H. Yildirim, D. F. Stamatialis, R. Ibanez, R. G. H. Lammertink, V. Jordan, and M. Wessling, Morphology and microtopology of cation-exchange polymers and the origin of the overlimiting current, The journal of physical chemistry. B 111, 2152 (2007). — p.94.

[17] S. M. Davidson, M. Wessling, and A. Mani, On the Dynamical Regimes of Pattern-Accelerated Electroconvection, Scientific Reports 6, 1 (2016). p.94.

[18] J. C. de Valença, R. M. Wagterveld, R. G. H. Lammertink, and P. A. Tsai, Dynamics of microvortices induced by ion concentration polarization, Physical Review E 92, 031003 (2015). — p.94, 97, 102, 104, 106.

[19] B. Baranowski and A. Kawczyński, Experimental determination of the critical rayleigh number in electrolyte solutions with concentration polarization, Electrochimica Acta 17, 695 (1972). — p.94, 99, 101.

[20] W. J. Ward and O. H. L. Blanc, Rayleigh-Benard Convection in an Electrochemical Redox Cell, No. 4669 in New Series, Science 225, 1471 (1984). - p.101.

[21] V. M. Volgin and A. D. Davydov, Natural-convective instability of electrochemical systems: A review, Russian Journal of Electrochemistry 42, 567 (2006). - p.94, 101.

[22] V. Fleury, J.-N. Chazalviel, and M. Rosso, Theory and experimental evidence of electroconvection around electrochemical deposits, Phys. Rev. Lett. 68, 2492 (1992). - p.94.

[23] M. Rosso, J.-N. Chazalviel, V. Fleury, and E. Chassaing, experimental evidence for gravity induced motion in the vicinity of ramified electrodeposits, Electrochimica Acta 39, 507 (1994). - p..

[24] G. Marshall, E. Mocskos, F. Molina, and S. Dengra, Three-dimensional na- 
ture of ion transport in thin-layer electrodeposition, Physical Review E 68, 021607 (2003). - p.94.

[25] C. W. Tobias, M. Eisenberg, and C. R. Wilke, Diffusion and convection in electrodialysis - A theoretical review, Journal of the Electrochemical Society 99, 359 (1952). - p.94.

[26] H. Rösler, F. Maletzki, and E. Staude, Ion transfer across electrodialysis membranes in the overlimiting current range: chronopotentiometric studies, Journal of membrane science 72, 171 (1992). — p.101.

[27] V. I. Zabolotsky, V. V. Nikonenko, and N. D. Pismenskaya, On the role of gravitational convection in the transfer enhancement of salt ions in the course of dilute solution electrodialysis, Journal of Membrane Science 119, 171 (1996). - p.94.

[28] E. Karatay, M. B. Andersen, M. Wessling, and A. Mani, Coupling between Buoyancy Forces and Electroconvective Instability near Ion-Selective Surfaces, Physical Review Letters 116, 194501 (2016). — p.94, 101.

[29] J. Hohlbein, M. Steinhart, C. Schiene-Fischer, A. Benda, M. Hof, and C. G. Hübner, Confined diffusion in ordered nanoporous alumina membranes, Small 3, 380 (2007). - p.97.

[30] G. Scalia and F. Scheffold, Lifetime of fluorescent dye molecules in dense aqueous suspensions of polystyrene nanoparticles, Optics Express 23, 29342 (2015). - p.97, 98 .

[31] J. R. Lakowicz, Principles of fluorescence spectroscopy, 3rd ed. (Springer, 2013). - p.98.

[32] P. Sistat and G. Pourcelly, Chronopotentiometric response of an ionexchange membrane in the underlimiting current-range. Transport phenomena within the diffusion layers, Journal of Membrane Science 123, 121 (1997). - p.98.

[33] D. E. Lide, CRC Handbook of Chemistry and Physics, 76th ed. (CRC Press, 1996), pp. 5-90. - p.98.

[34] V. V. Nikonenko, N. D. Pismenskaya, E. I. Belova, P. Sistat, P. Huguet, G. Pourcelly, and C. Larchet, Intensive current transfer in membrane systems: Modelling, mechanisms and application in electrodialysis, Advances in Colloid and Interface Science 160, 101 (2010). — p.99.

[35] F. Maletzkl, H. Rdsler, E. Staude, F. Maletzki, H.-W. Rösler, and E. Staude, Ion transfer across electrodialysis membranes in the overlimiting current range : stationary voltage current characteristics and current noise power spectra under different conditions of free convection, Journal of Membrane Science 71, 105 (1992). - p.99.

[36] S. Hanasoge and F. J. Diez, Vortex Chain Formation in Regions of Ion Concentration Polarization, Lab Chip (2015). - p.99. 
[37] M. Rosso, E. Chassaing, and J.-N. Chazalviel, Role of buoyancy in the onset of dendritic growth in thin layer electrodeposition, Physical Review E 59, 3135 (1999). - p.101.

[38] N. D. Pismenskaya, V. V. Nikonenko, E. I. Belova, G. Y. Lopatkova, P. Sistat, G. Pourcelly, and K. Larshe, Coupled convection of solution near the surface of ion-exchange membranes in intensive current regimes, Russian Journal of Electrochemistry 43, 307 (2007). — p.101.

[39] E. M. Sparrow, R. J. Goldstein, and V. K. Jonsson, Thermal instability in a horizontal fluid layer: effect of boundary conditions and non-linear temperature profile, Journal of Fluid ... 18, 513 (1964). — p.101.

[40] R. Kwak, G. Guan, W. K. Peng, and J. Han, Microscale electrodialysis: Concentration profiling and vortex visualization, Desalination 308, 138 (2013). — p.101.

[41] A. J. Bard and L. R. Faulkner, Methods, 2nd ed. (2001), pp. xxi, 833 p. - p.102.

[42] M. Van Soestbergen, P. M. Biesheuvel, and M. Z. Bazant, Diffuse-charge effects on the transient response of electrochemical cells, Physical Review E - Statistical, Nonlinear, and Soft Matter Physics 81, 1 (2010). — p.102.

[43] J. Krol, M. Wessling, and H. Strathmann, Chronopotentiometry and overlimiting ion transport through monopolar ion exchange membranes, Journal of membrane science 162, 155 (1999). - p.102, 106.

[44] S. A. Mareev, D. Y. Butylskii, N. D. Pismenskaya, and V. V. Nikonenko, Chronopotentiometry of ion-exchange membranes in the overlimiting current range. Transition time for a finite-length diffusion layer: Modeling and experiment, Journal of Membrane Science 500, 171 (2016). — p.103. 


\section{CHAPTER 5}

\section{Confined electroconvective vortices at structured membranes}

This chapter presents the visualization of electroconvective ion transport at cation exchange membranes with different geometrical square-wave structures (line undulations). A constant voltage difference is applied across the membrane which causes the ion concentration at the interface to deplete after which electroconvective micro-vortices appear once a threshold voltage drop is applied. The potential drop needed to start and sustain electroconvection decreases with $50 \%$ when the structure size is similar to the mixing layer thickness which results in confined vortices with less lateral motion compared to at flat membranes. From electrical, flow and concentration data, ion migration, advection and diffusion ion transport are quantified. Advection and migration are dominant in the vortex mixing layer and diffusion and migration are dominant in the stagnant diffusion layer. The fluxes have the same magnitude, therefore indicating no other major transport phenomena are expected to be present. Presented numerical simulations show similar ion transport behaviour by employing an electro-osmotic slip boundary condition. The only difference is the direction of rotation of the vortices at the structured surface, leaving the question of the exact coupling mechanism at the interface unanswered.

This chapter is in preparation to be submitted for publication as:

Confined electroconvective vortices at structured membranes enhance overlimiting current, Joeri C. de Valença, Morten Jõgi, R. Martijn Wagterveld, Elif Karatay, Ali Mani, Jeffery A. Wood, Rob G. H. Lammertink, Physical Review Fluids (2017) 
Electroconvection in electrolytes can occur when the electric field stands perpendicular to a charge selective interface (ion exchange membrane [1], electrode [2], micro-nano channel [3]) and a critical voltage is applied. On one hand, this allows faster ion transport than diffusion alone near the interface, thereby possibly enhancing the desalination rate in electrodialysis [4] or electrodeposition speed [5]. On the other hand, current efficiency drops and additional processes might occur like water dissociation [4] or complex dendrite formation [5].

The selectivity of a cation exchange membrane (CEM) allows cations to permeate through while rejecting the anions that migrate in the opposite direction. The ion flux imbalance at the interfaces results in concentration change: at one side the concentration enriches, at the other side it depletes. The expansion of the concentration change into an ion gradient in the aqueous solution is called ion concentration polarization (ICP). When the interface concentration at the depleted side approaches zero, the high electrical resistance of this thin interface layer becomes dominant in the overall resistance. This situation is called the limiting current condition $\left(i_{\lim }\right)$ and it is seen as the upper limit of practical application in electrodialysis [6]. If the voltage is increased the high resistance layer becomes unstable and additional current goes through, called overlimiting current (OLC). The occurrence of OLC is known for more than 50 years $[7,8]$. Several physical and chemical mechanisms have been proposed to describe OLC, but interfacial mixing of the depleted boundary layer due to electroconvection (electo-osmosis) is the main candidate $[1,4,9]$ and previous chapters.

Different electro-osmotic coupling mechanisms exist [10]. Dukhin explored the coupling of an electric field along a curved charged surface in an electrolyte and described the formation of an extended space charge layer (ESCL) [11, 12]. This layer forms when the concentration is depleted out of equilibrium by an external electric field. Rubinstein and Zaltzman worked out a theory how the ESCL develops at a flat membrane with perpendicular electric field. They found that the ESCL could become unstable such that a small perturbation starts electro-osmosis (EO) perpendicular to the applied electric field $[9,13,14]$. The EO drives vortices which creates a positive feedback loop in which the vortices enhance the initial inhomogeneity. This instability mechanism is referred to as the electro-kinetic instability (EKI) $[15,16]$. At a suitable undulated surface both electroconvective mechanisms can occur. Rubinstein predicted that an undulated surface decreases the energy needed to sustain the vortices if the mixing layer is in the same order as the undulations. $[9,17]$. Recent direct numerical simulations by Davidson et al. [18] investigated a patterned surface consisting of patches of conducting and non-conduction surface. The research predicted an increase in ion transport rate of $80 \%$ when the pattern size is similar to the mixing layer thickness. 
Physical experiments with geometrically structured membranes showed a $60 \%$ decrease in voltage needed to start OLC [19]. Later also chemical patterned membranes were found to cause a reduction in onset [20]. The chemical degradation of membrane were also found to induce inhomogeneities in the surface that enhances the onset of electroconvection [21]. Also the hydrophobocity of the surface influences the efficiency of the electroconvection [22]. This work attempts to elucidate on the mechanisms by visualizing the flow and concentration field next to a membrane with periodic line structures. The current efficiency of electroconvective mixing at a cation exchange membrane is found to have an optimum when the membrane undulations are the same size as the mixing layer.

\subsection{Material and methods}


Figure 5.1: A SPEEK solution is casted on a mold with different structures. After the solution solidifies it is released. Membranes with $L=50,100,200,400$ and $800 \mu \mathrm{m}$ and $H=50 \mu \mathrm{m}$ were made as well as a flat membrane. Image (4) shows a SEM photo of a structured membrane with $L=400 \mu \mathrm{m}$. The dotted line represents the edge of the flat side. 


\subsubsection{Membrane preparation and pretreatment}

The cation exchange membranes are composed of $80 \mathrm{wt} . \%$ sulfonated poly ether ether ketone (SPEEK) mixed with 20 wt.\% glycerol. To synthesize the membranes SPEEK (67\% sulfonation degree), glycerol, ethanol and ultrapure water (MilliQ) were used. The synthesis of the membranes is based on evaporation. A mixture of $40 \mathrm{~g}$ SPEEK and $8 \mathrm{~g}$ glycerol is solved in $160 \mathrm{~g}$ water:ethanol $(1: 1)$. The solution is poured in a pattern-etched mold and then sonicated in an ultrasound bath for 15 minutes so the polymeric mixture becomes properly distributed and also to release trapped air bubbles. After the ultrasonic bath it is placed under $\mathrm{N}_{2}$ atmosphere for $24 \mathrm{~h}$, after which is can be peeled of the mold. The mold was made using a photolithography process in the cleanroom (University Twente). The process is similar to what was described by Balster [19]. The structures where designed to be rectangular ridges with an extrusion height (H) of $40 \mu \mathrm{m}$ and varying width (L): 50, 100, 200, 400 and $800 \mu \mathrm{m}$, see figure 5.1. The gap has the same width as the extrusion and the length of the lines is $4.5 \mathrm{~mm}$.

Before the measurement the membranes have to be brought into equilibrium with the electrolyte solution used for the measurements, $10 \mathrm{mM} \mathrm{CuSO}_{4},\left(\mathrm{CuSO}_{4}\right.$. $5 \mathrm{H}_{2} \mathrm{O}$ (VWR Chemicals) and ultrapure water (milliQ)). To ensure the membrane counter-ions are $\mathrm{Cu}^{2+}$ and the co-ions $\mathrm{SO}_{4}{ }^{2-}$ a new membrane is first immersed in a $100 \mathrm{mM} \mathrm{CuSO}_{4}$ solution for 24 hours. After this the membrane is rinsed with ultrapure water and the excess water is wiped off. This step is conducted twice. Afterwards the membrane is placed in the measurement solution for 24 hours. The solution is changed three times to ensure the membrane is in equilibrium with the measurement solution.

\subsubsection{Experimental setup}

Figure 5.2 shows the experimental setup with a structured membrane, $A_{\text {mem }}=$ $3 \times 4.5 \mathrm{~mm}^{2}$, placed between two reservoirs filled with a $10 \mathrm{mM} \mathrm{CuSO}_{4}$ electrolyte solution, both enclosed by two copper electrodes, the cathode and andode. When a voltage difference is applied between these two electrodes by a potentiostat (Autolab PGSTAT 30) redox reactions transfer the electric current to ionic current [23]. The anode, where copper oxidizes, serves as a $\mathrm{Cu}^{2+}$ cation source, and the cathode, where copper reduces, acts as a $\mathrm{Cu}^{2+}$ cation sink, allowing for steady state current in the stagnant electrolyte. Two copper wires are inserted at both sides of the membrane (0.6 $\mathrm{mm}$ from membrane in anode compartment, $7.3 \mathrm{~mm}$ from membrane in cathode compartment) and act as sense electodes. At these electrodes the same redox reactions happen as at the anode and cathode, but the 


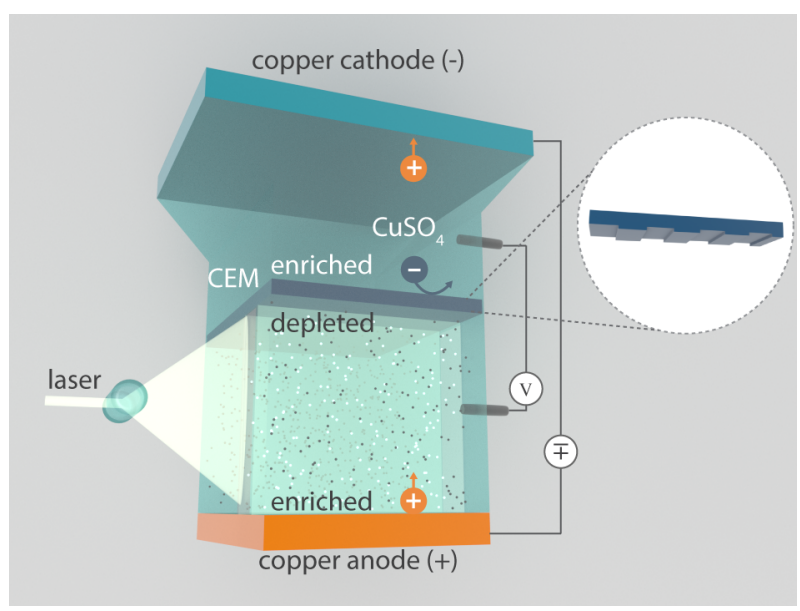

Figure 5.2: Structured membrane between the anode and cathode compartment filled with $\mathrm{CuSO}_{4}$ solution. Simultaneous to the electrical measurements the flow dynamics are captures by imaging the displacement of suspended particles.

amount of current is too low to disturb the concentration significantly. With a feedback loop the voltage drop between the sense electrodes is kept at $\Delta V=1 \mathrm{~V}$ and the time-dependent current between the anode and cathode is measured, $\Delta I(t)$. Since the current is continuous the time-dependent resistance between the sense electrodes can be calculated, $R(t)=V / I(t)$.

Flow dynamics in the anode compartment are measured by seeding the solution with $0.1 \mathrm{wt} \% 2 \mu \mathrm{m}$ red polystyrene tracer particles (Microparticles $\mathrm{GmbH}$, with a density of $\left.1.05 \mathrm{~g} / \mathrm{cm}^{3}\right)$. The particles are illuminated with a thin $(\approx 0.2 \mathrm{~mm})$ laser sheet (808 nm, Firefly, Oxford lasers), see figure 5.2, and the reflected light is captured through a long distance magnifying lens $(2-7 \times$, Navitar) on a camera at 10 frames per second. From the recorded particle displacement the vector field is determined using particle image velocimetry (PIV) analysis (DaVis, LaVision) following the same algorithms as described in Chapter 4. The coordinate system is taken such that $x$ represents the path between membrane and anode, the height in the plane, $y$ the path along the membrane and $z$ the depth into the cell.

The concentration variation is measured using fluorescent lifetime image microscopy (FLIM), (LIFA, Lambert instruments) by adding $2.5 \mu \mathrm{M}$ fluorescent dye (Alexa Fluor ${ }^{\circledR} 488$ Cadaverine, Life technologies) to the solution. The fluorescence decay of the dye depends on the $\mathrm{CuSO}_{4}$ concentration in the range between 1 and $100 \mathrm{mM}$ (see section 2.3.1 for calibration curve). A modulated blue LED light is sent through a $5 \mathrm{x}$ magnifying objective (Zeiss) and the green fluorescent 
light is captured on a $696 \times 520$ pixels CCD camera. Each pixel represents an area of $4.17 \times 4.17 \mu \mathrm{m}^{2}$.

\subsection{Results and discussion}

\subsubsection{The onset of electroconvection}

Each measurement starts by applying a constant voltage drop of $1 \mathrm{~V}$ between the reference electrodes on both sides of the membrane with an initial stagnant and homogeneous solution, $v=0$ and $\nabla c(x)=0$. The initial ohmic resistance $R_{\text {ini }}=V /\left(i \cdot A_{\text {mem }}\right)=5.0 \pm 0.2 \mathrm{k} \Omega$ does not depend on membrane type, indicating that the electrolyte resistance is dominant. The initial resistance corresponds to the expected resistance based on the conductivity of $10 \mathrm{mM} \mathrm{CuSO}_{4}(1.5 \mathrm{mS} / \mathrm{cm})$ in the channel geometry.

Due to the selectivity of the membrane and anode, cations migrate in and out the anode compartment, while the anions, migrating the opposite direction, are blocked. The discontinuity in ion flux at the interfaces will lead to a change in ion concentration following, $\nabla \cdot J_{i}=-\partial c_{i} / \partial t$. The interface concentration gradient will grow due to diffusional ion transport, $J_{d i f}=-D \nabla c$. This process is called ion concentration polarization and can be directly observed from the change in fluorescent lifetime of the dye due to the change in concentration (see figure 5.7 and supplemental movie). Since the resistance is inversely proportional to the concentration, the resistance of the depleted side will dominate over the enriched side where the resistance goes down. The concentration difference at the two sides of the membrane also results in a potential difference called the Nernst potential, as the membrane is ion selective. This potential difference is typically much smaller than the applied potential difference, $V_{n}=\left[k_{\mathrm{B}} T\right] /[z e] \ln \left[c_{1} / c_{2}\right] \approx 70 \mathrm{mV}$ for $c_{1}=0.1 \mathrm{mM}$ and $c_{1}=20 \mathrm{mM}$. The first $\approx 40 \mathrm{~s}$ of figure 5.3 show an increase in voltage.

When the concentration at the interface approaches zero, the resistance in the thin interfacial layer dominates leading to a sharp increase as seen in the inset in figure 5.3. The depletion time, $\tau_{\mathrm{c}}$, is taken as the first point where the rate of change of the resistance gradient is maximum $\left(\partial_{t}^{3} R(t)=0\right)$. The different membranes show no trend regarding their $\tau_{c}=42 \pm 5 \mathrm{~s}$ and $R\left(\tau_{\mathrm{c}}\right)=5.7 \pm 0.2 \mathrm{k} \Omega$. The second point where $\partial_{t}^{3} R(t)=0$ marks the start of electroconvective mixing of the interface layer. This second transition time, $\tau_{\mathrm{EC}}$, coincides with the first particle motion at the membrane in the optical measurements.

Although no trend was observed between the $\tau_{\mathrm{EC}}=60 \pm 7 \mathrm{~s}$ at the different membranes, however in $R\left(\tau_{\mathrm{EC}}\right)$ it was observed. The jump in resistance is defined as $R_{\text {jump }}=R\left(\tau_{\mathrm{EC}}\right)-R\left(\tau_{\mathrm{c}}\right)$ and reduces with the presence of the structures, 

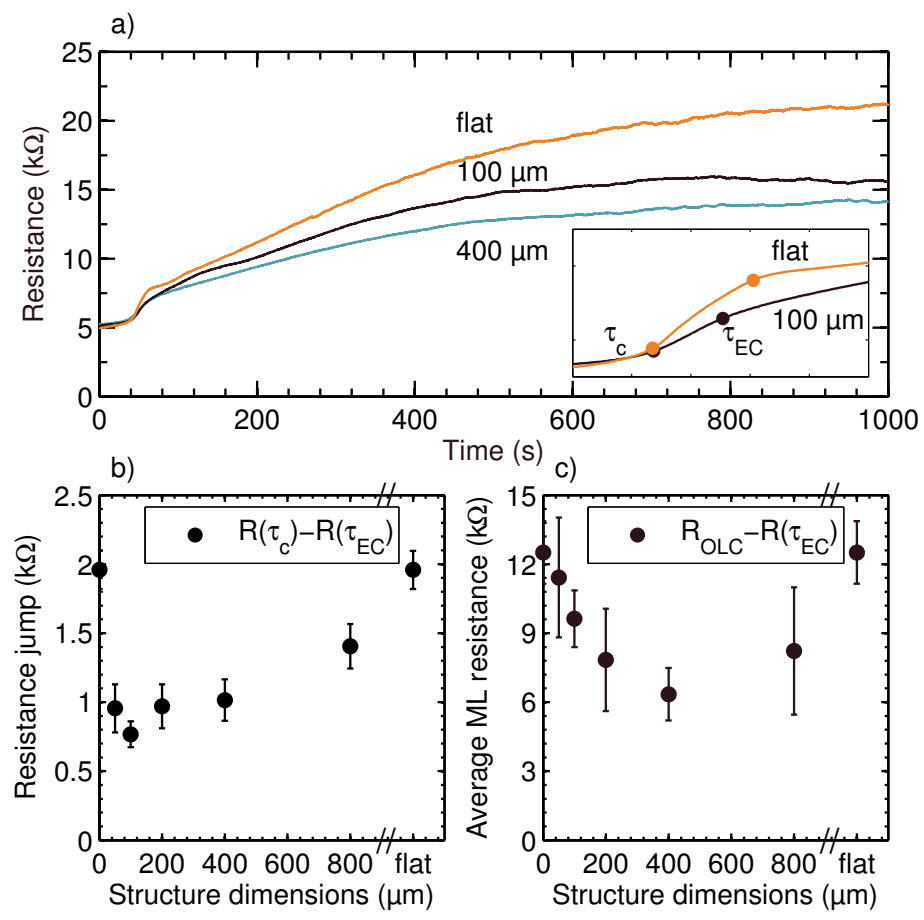

Time (s) $\mathrm{c})$

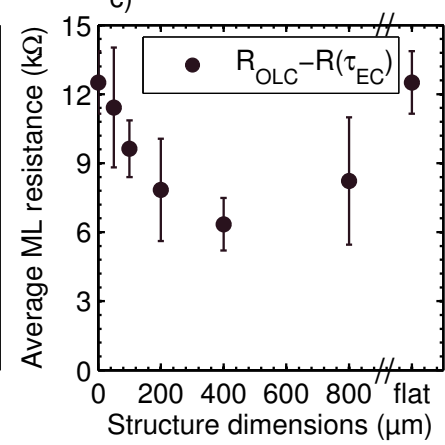

Figure 5.3: a) The resistance changes in time after applying $\Delta V=1 \mathrm{~V}$ over different membranes. b) The resistance jump $\left(R_{\text {jump }}\right)$ indicates the threshold voltage drop needed to start electroconvection. The errorbar represents the standard deviation from multiple experiments. The value of the flat membrane has been places at the beginning and the end of the $x$ axis, since it can be seen as a zero or infinite structure dimension. c) The saturated resistance $\left(R_{\mathrm{sat}}\right)$ is calculated by taking the average resistance value over the last 400 seconds $\left(R_{\mathrm{OLC}}\right)$ and subtract $\left(R\left(\tau_{\mathrm{EC}}\right)\right)$. This assumes the additional resistance increase after OLC starts is caused by the mixing layer. The errorbar represents the standard deviation from multiple experiments.

see figure $3 \mathrm{~b}$. The errorbar represents the standard deviation over three to six measurements, some with and some without particles. The presence of structures diminishes the energy needed to start the electroconvective mixing with a minimum at the membrane with $100 \mu \mathrm{m}$ undulations, having a $60 \%$ reduction compared to the flat membrane. This results can be interpreted as a shortening of the plateau length in a typical IV curve as seen by Balster [19] and is in line with numerical predictions of Rubinstein [9]. 


\subsubsection{The growth of electroconvection}

Structures on the membrane affect the onset of the electroconvective flow, as seen in figure 5.4a-c. A detailed study on the development of the different type of observed electroconvective vortices paves the way for an explanation to what type of mixing occurs and which has the lowest resistance. First the general properties of the electroconvective mixing are described followed by the difference between mixing at a flat membrane versus mixing at a structured membrane.

The layer with advection is called the mixing layer (ML), while the consequently shrinking stagnant layer is called the diffusion layer (DL). Similar two layer separation of the system has been seen before [24] and predicted numerically [25]. The FLIM results confirm that in the DL a concentration gradient is present, while the $\mathrm{ML}$ has a low concentration, $\mathcal{O}(1 \mathrm{mM})$, see figure 5.7. The advective transport mechanism brings both anions and cations towards the membrane, allowing faster than diffusive transport.

Once the vortices are big enough $(\approx 0.3 \mathrm{~mm})$ the boundary of the mixing layer is derived from the PIV results, following the same algorithm as in Chapter 4. The vortex boundary threshold is taken as $20 \%$ of the maximum rms velocity. This threshold value underestimates the mixing layer thickness, but is less prone to erroneous vectors. The edge of the membrane is taken as the edge of the extrusion. Initially the height of the mixing layer and the average root mean square (rms) velocity within the layer grow in time, see figure 5.5. The resistance grows in a similar way, see figure $5.3 \mathrm{a}$, which leads to the assumption that the additional resistance mainly comes from the growing mixing layer which has a low average concentration, thus a low conductivity. This additional resistance is calculated as $R_{\mathrm{OLC}}=R_{\mathrm{tot}}-R_{\mathrm{ohmic}}-R_{\text {jump }}$. It remains an open question if the voltage drop near the membrane surface remains the same while the vortices grow. In other words, does the initial resistance increase $\left(R_{\text {jump }}\right)$ keeps the same value although larger vortices are driven by electro-osmosis coupling?

At the flat membrane the first particle motion (around $\tau_{\mathrm{EC}}$ ) occurs at the membrane at apparently random locations. The particle pathlines reveal small vortices that grow and merge with other vortices, while displaying lateral movement along the surface. The mean rms velocity inside the mixing layer grows similar to what was observed in Chapter 3 and 4.

At the structured membrane the vortices also grow and merge, but often show a periodic flow profile, where the vortices have preferred locations. Repeating the experiments at different positions $(\Delta z \approx 1.5 \mathrm{~mm})$ shows similar behaviour. This leads to the hypothesis that the $3 \mathrm{D}$ behaviour of the mixing occurs as vortex rolls along the ridges. Typically the flow is directed to the corners of the structures with inflow from above the gap and outflow above the extrusion. This is 

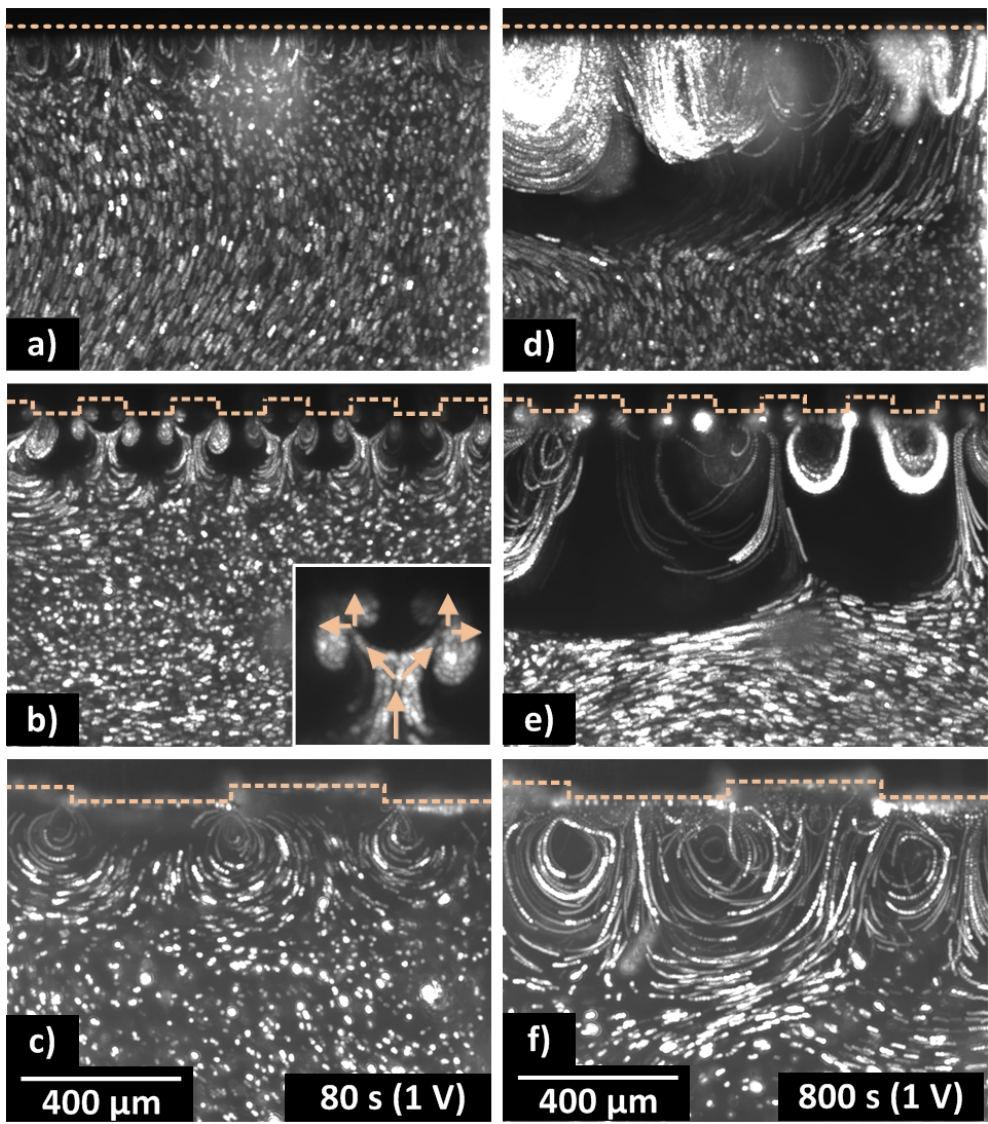

Figure 5.4: Overlaying 100 images shows the particle pathlines over $10 \mathrm{~s}$. a) flat, b) $100 \mu \mathrm{m}$, c) $400 \mu \mathrm{m}(70-80 \mathrm{~s})$ During the growth phase the structures influence the electroconvective vortex shape. d) flat, e) $100 \mu \mathrm{m}, \mathrm{f}) 400 \mu \mathrm{m}(790-800 \mathrm{~s})$. At a flat membrane the electroconvective vortices are moving along the membrane. At the membrane with ridges of $400 \mu \mathrm{m}$ the vortices are stable. At a membrane with $100 \mu \mathrm{m}$ ridges voids empty of particles are formed, while the particle density at the surface increases locally. The membrane edge is indicated with a dotted line.

opposite to theoretical prediction at sinusoidal curved membranes $[9,15]$. At the structured membranes typically two types of vortices appear, as shown in figure $5.4 \mathrm{~b}$. The inset shows an the inner vortex along the surface parallel to the electric field, and the outer vortex along the surface perpendicular to the electric field. Most probably the inner vortex is driven by electro-osmotic wall flow (EOF), also referred to as the Dukhin mode. While the outer vortices are probably driven by 


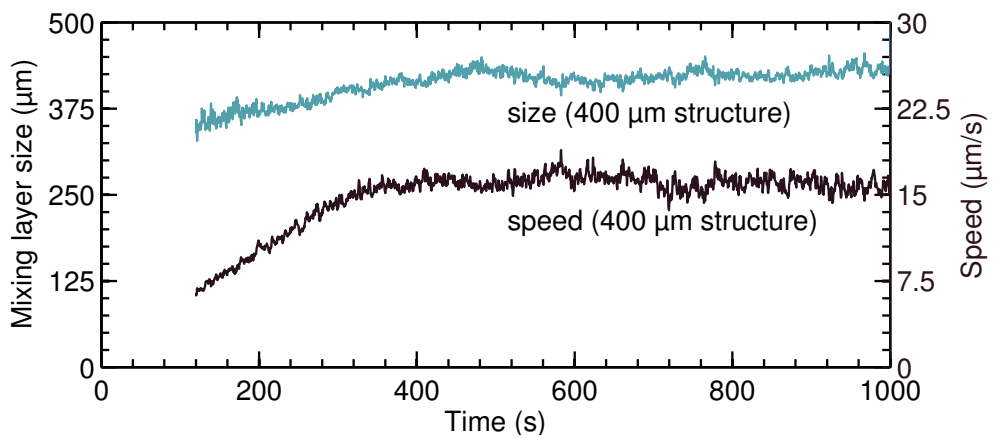

Figure 5.5: a) The mixing layer thickness (vortex size) and average rms velocity within the mixing layer (vortex speed) grow in time till a saturated size and speed.

the electro-kinetic instability, also referred to as the Rubinstein mode.

The inner vortices are visible as flow pockets where particles are trapped. The diameter of the vortices does not grow bigger than $\approx 50 \mu \mathrm{m}$ and the velocities inside can go up to $100 \mu \mathrm{m} / \mathrm{s}$. The aggregation of particles, the presence of the membrane, and optical resolution of the system makes it challenging to determine the velocities with high precision. The opposite effect of trapping is the depletion of particles at the edge of the mixing layer. Whether the particle motion deviates from the fluid streamlines and moves towards the vortex centre depends on the body forces on the particle. A decrease in momentum, due to inertia effects like stokes drag typically directs the particles away from the vortex centre, while electric forces direct it inward $[18,26,27]$. Inertial effects are likely not of any role here, but a precise force balance is out of the scope of our research, but could be used to estimate the electrical field gradient inside the mixing layer.

The outer vortices grow up to $\approx 0.5 \mathrm{~mm}$ and displace the depleted interface layer near the membrane with a higher concentrated solution. The width of the outer vortices is about the same as their height. When the width of the vortices is similar to the width of the structures periodic flow patterns are observed. If the vortex width grows larger than the structure dimension the periodic flow profile breaks down, as seen in figure 5.4e. Vortices seem to hold their position at the membrane between the ratio $L_{m i x}=0.5 \cdot L_{m e m}$, where they are elongated ellipses along the membrane (fig 5.4c), and $L_{m i x}=2 \cdot L_{m e m}$, where they are elongated ellipses from the membrane (fig 5.4b). When the vortices are spherical, $L_{m i x} \approx L_{m e m}$ the void area is lowest. At the larger structures $(400$ and $800 \mu \mathrm{m})$ the vortices occur only at the corners of the structures. With the growth of the mixing layer these vortices do not need to merge while growing to the saturated size. 
There is a natural ratio between the vortex size and structure size which keeps the vortices confined and stable. The resistance of the mixing layer with steady vortices is always lower than with lateral moving vortices. The lateral movement does not enhances the ion transport towards the membrane. Confined vortices are more effective in mixing the high and low concentration, compared to the ones with the lateral motion. This matches results found in recent numerical work [18].

\subsubsection{The saturation of electroconvection}

The mixing layer grows to $\approx 0.5 \mathrm{~mm}$ with saturation time around $t \approx 600 \mathrm{~s}$ as can be seen from the particle pathlines, see figure $5.4 \mathrm{~d}-\mathrm{f}$, and the PIV results in figure 5.5. The mixing layer is most stable when the structures are $400 \mu \mathrm{m}$ and the lateral movement that is typical at the flat membranes does not occur. Also when the structures are 200, and $800 \mu \mathrm{m}$ the vortices are well confined and the mixing layer size is bigger, see figure $5.6 \mathrm{~b}$. The conductivity of the saturated mixing layer is calculated via $\sigma=L_{\text {mix }} /\left(R_{\text {sat }} \cdot A_{\text {mem }}\right)$, where $R_{\text {sat }}$ is the average resistance in the mixing layer from $\mathrm{t}=600 \mathrm{~s}$ to $\mathrm{t}=1000 \mathrm{~s}$, and $L_{\text {mix }}$ is the average length in this period. The conductivity shows a maximum for structures that have the same width as the ML thickness which makes the vortex location confined, see figure 5.6c.

The formation of particle voids complicates the extraction of the flow field and the vectors are interpolated or extrapolated from the regions with sufficient amount of particles. The mixing layer with the $50 \mu \mathrm{m}$ structured membrane has too much void formation to extract reliable PIV vector fields. The edge of the void was also determined as a measure of the mixing layer thickness, see figure $5.4 \mathrm{~d}$-e and $5.6 \mathrm{~b}$. The edge was determined by horizontally averaging the pixel intensity and track at what distance from the membrane the intensity sharply increases. A similar approach is used to determine the vortex edge in the vector field data. At the $400 \mu \mathrm{m}$ membrane hardly any void formation is seen, so no void boundary could be extracted, see figure $5.4 \mathrm{f}$ and $5.6 \mathrm{~b}$. Increasing the structure size $(800 \mu \mathrm{m})$ increases the void area, but still less than at the flat membrane. Decreasing the structure size $(200 \mu \mathrm{m}$ to $50 \mu \mathrm{m})$ increased the void area as well, but all have a larger void area than at the flat membranes. Increasing the structure number increases the number of inner vortices which trap the tracer particles, therefore decreasing the particle density in the outer vortices.

The FLIM measurements confirm that the average concentration inside the mixing layer is higher with stable vortices in case of $800 \mu \mathrm{m}$ vs flat, see figure 5.7. Time averaging $(t=600-1000 \mathrm{~s})$ of the concentration field proves also that the presence of the structures hinders the lateral motion allowing the formation 

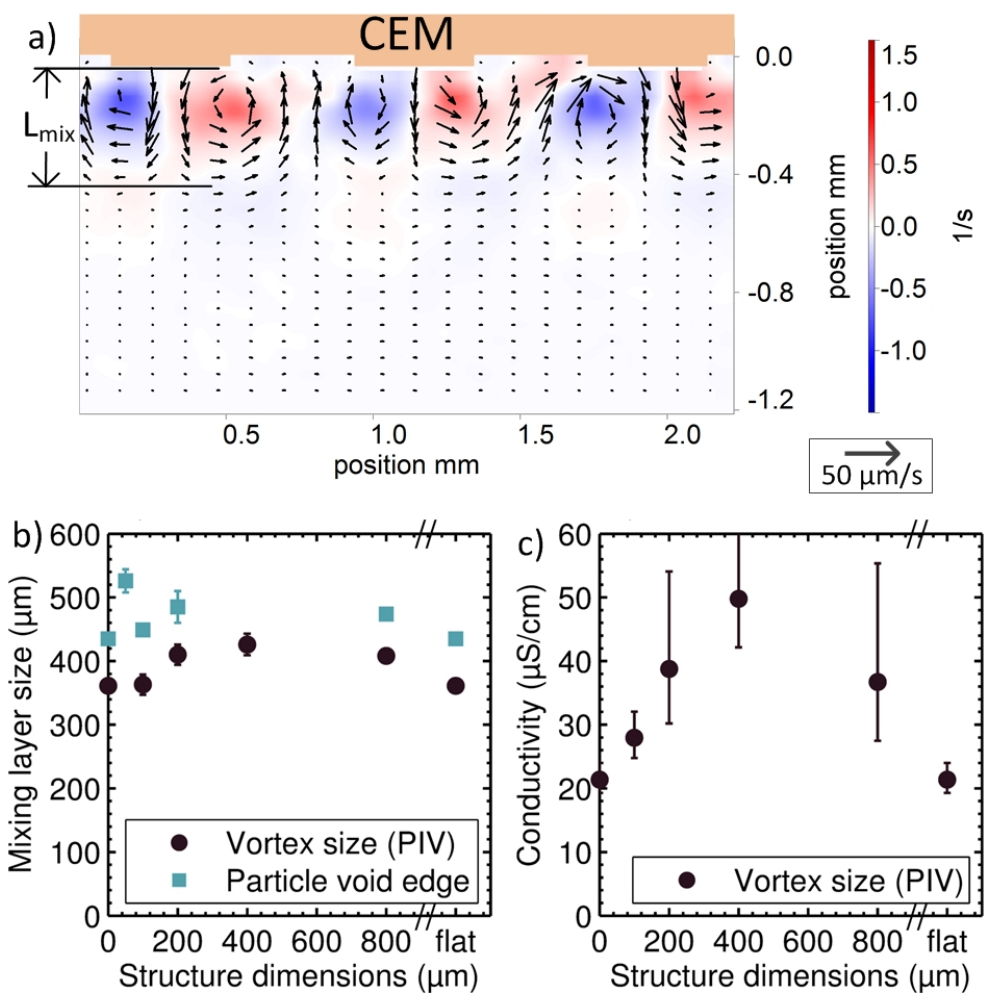

Figure 5.6: a) The steady vortices at the $400 \mu \mathrm{m}$ are resolved with PIV. Shown is the average motion between 700-710 s (100 image pairs). The vortex boundary was taken as the place where the rms velocity was $20 \%$ of the maximum. $L_{m i x}=426 \mu \mathrm{m}$ with $v_{r m s}=17 \mu \mathrm{m} / \mathrm{s}$. The background colour represents the vorticity of the vector field. Every second row of vectors is omitted for visibility. b) The mixing layer size is determined by PIV (as describe above) and from the edge of the particle void (by taken the horizontal average of the light intensity). c) The conductivity within the mixing layer is calculated via $\sigma=L_{\mathrm{mix}} /\left(R_{\mathrm{sat}} \cdot A_{\mathrm{mem}}\right)$.

of stable conduction channels. The concentration data is also in line with the flow results that the flow towards the membrane is located near the gap, having a higher concentration, while the flow away from the membrane is located above the extrusion, having a lower concentration. A time-averaged vector field $(\mathrm{t}=600$ $800 \mathrm{~s}$ ) from a separate $1 \mathrm{~V}$ measurement overlays the concentration profile in figure 5.7b. This is not done for the flat membrane since the flow dynamics are not stable over $200 \mathrm{~s}$. Also at $100 \mu \mathrm{m}$ structured membranes no conduction channels were observed. This can be due to the limited spatial and temporal 
resolution of the FLIM system, but also small thin vortices will have higher lateral concentration gradient, therefore diffusion (dispersion), which smooth out the concentration gradients [28].

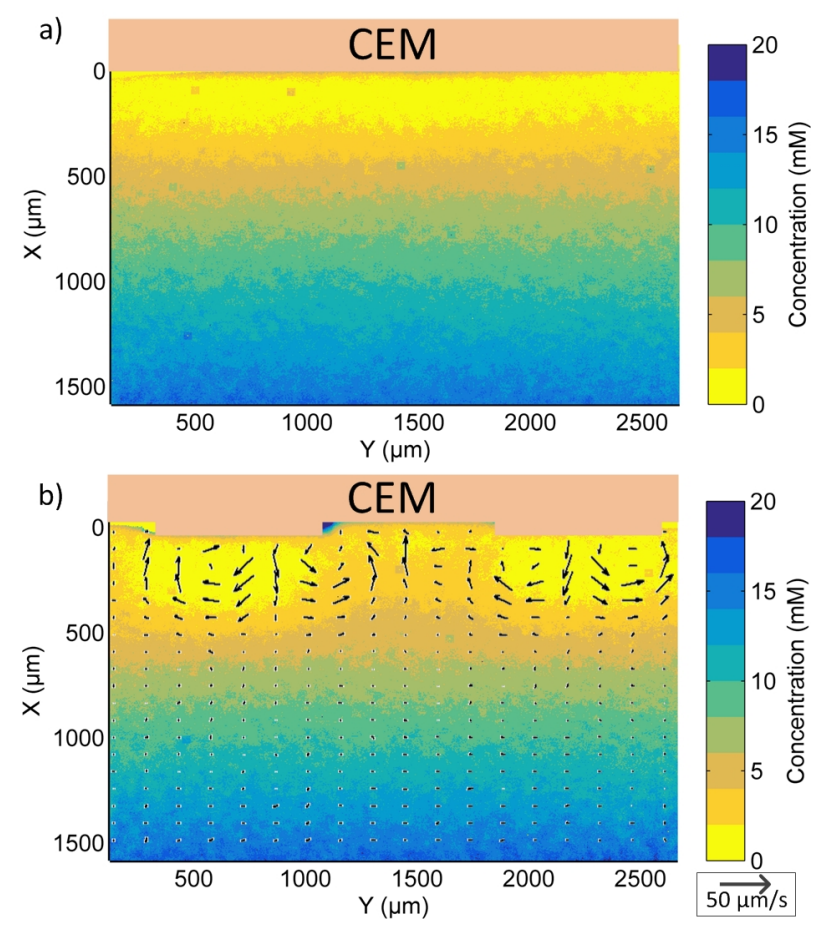

Figure 5.7: Concentration field (via FLIM) near a flat and structured ( $800 \mu \mathrm{m})$ membrane in the saturated regime. The concentration field is a time-average field $(t=600-$ $1000 \mathrm{~s}$ ) constructed from 33 separate lifetime images. On top of the concentration field at the $800 \mu \mathrm{m}$ membrane an overlay of the time averaged flow field (via PIV) is shown $(\mathrm{t}=600-800 \mathrm{~s})$. The flow field is derived from a separate experiment with similar electrical response. At the flat membrane also an electroconvective mixing layer is present, but due to the unsteady lateral motion of the vortices the time average flow field is not representative for the motion within the layer.

\subsubsection{Ion flux calculations}

Having the current, flow and concentration data makes it possible to estimate the electric, diffusive and advective fluxes in the system. A one dimensional approach is taken to describe the average values perpendicular to the membrane (wall normal, $x$ direction). The measured current is assumed to be carried only by the 
$\mathrm{Cu}^{2+}$ and $\mathrm{SO}_{4}{ }^{2-}$ and electroneutrality is assumed in the bulk. The theoretically extended space charge layer is expected to be about $10 \times$ the Debye length $[14,29]$, in this system $\lambda_{D} \approx 10^{-8} \mathrm{~m}$. Numerical simulations showed that in the chaotic regime charged regions do occur, but that they do not contribute to the overall ion advective transport $[25,30]$. The ion transport is described by a modified Nernst-Planck equation (see [8] and Chapter 1 for more details):

$$
\begin{aligned}
J & =J_{\mathrm{el}}+J_{\mathrm{dif}}+J_{\mathrm{adv}} \\
J_{i} & =\frac{i t_{i}}{z_{i} F}-D \frac{d c_{i}}{d x}+c_{i} v \\
i & =\mathrm{Cu}^{2+}, \mathrm{SO}_{4}{ }^{2-}
\end{aligned}
$$

where the electric current density is $i=F\left(z_{+} J_{+}+z_{-} J_{-}\right)$. The transport number, $t_{i}$, reflects the part of the current carried by each species dependent on its individual diffusion coefficient, $t_{i}=D_{i} /\left(D_{+}+D_{-}\right)$. Inside the liquid $t_{+}=0.4$ and $t_{-}=0.6$. An ideal cation exchange membrane does not allow anions to pass, thus the $\overline{t_{+}}=1$ and $\overline{t_{-}}=0$. Faraday's constant is $F$ and $z_{+}=-z_{-}=2$ is the valence number of the ions. The ion concentration is $c_{i}$ and the total salt diffusion constant is $D=\left(z_{+}+\left|z_{-}\right|\right) D_{+} D_{-} /\left(z_{+} D_{+}+\left|z_{-}\right| D_{-}\right)=0.855 \cdot 10^{-9} \mathrm{~m}^{2} / \mathrm{s}[23]$. The velocity of the liquid in the direction of the electric field is $v$.

This analysis focuses on the anion flux inside the anode compartment in the saturated regime. Since the anions cannot pass the membrane and the anode, their total flux has to be zero. The FLIM concentration image (see figure 5.7) shows a linear concentration gradient along the electric field in in the stagnant layer. The diffusional flux can be estimated to be $J_{-}^{\text {dif }}=-D \partial c / \partial x=-D \Delta c_{\mathrm{DL}} / L_{\mathrm{dif}}=$ $1.1 \cdot 10^{-5} \mathrm{~mol} / \mathrm{m}^{2} \mathrm{~s}$, where $\Delta c_{\mathrm{DL}}=20 \mathrm{mM}$ and $L_{\mathrm{dif}}=L_{\mathrm{res}}-L_{\mathrm{mix}}$. This magnitude matches with an electromigration flux corresponding to an electric current density of $3.5 \mathrm{~A} / \mathrm{m}^{2}$, where the measured current density lies between $5.3 \mathrm{~A} / \mathrm{m}^{2}$ (optimal structures size) and $3.7 \mathrm{~A} / \mathrm{m}^{2}$ (flat membrane). With the growth of the mixing layer the concentration gradient in the stagnant layer increases, giving a higher diffusional transport rate. For comparison, the theoretical limiting current density is $2.7 \mathrm{~A} / \mathrm{m}^{2}\left(\Delta c_{\mathrm{DL}}=20 \mathrm{mM}\right.$ and $\left.L_{\text {dif }}=2 \mathrm{~mm}\right)$.

For the transport in the mixing layer, the diffusion in the direction of the electric field is neglected at first $\left(P e=v_{\mathrm{rms}} L_{\mathrm{mix}} / D \approx 7\right.$, with $v_{\mathrm{rms}}=12 \mu \mathrm{m} / \mathrm{s}$, $L_{\text {mix }}=0.5 \mathrm{~mm}$ ). For simplicity the mixing layer is separated in a part with upflow, indicated as $J^{\mathrm{adv} \uparrow}=c_{\uparrow} v_{\uparrow}=6 \cdot 10^{-5} \mathrm{~mol} / \mathrm{m}^{2} \mathrm{~s}$, and a part with downward flow, $J^{\mathrm{adv} \downarrow}=c_{\downarrow} v_{\downarrow}=-2 \cdot 10^{-5} \mathrm{~mol} / \mathrm{m}^{2} \mathrm{~s}$, where $v=20 \mu \mathrm{m} / \mathrm{s}, c_{\uparrow}=3 \mathrm{mM}$ and $c_{\downarrow}=1 \mathrm{mM}$. These values are derived from the PIV and FLIM results, figure 5.7b. This leads to $J^{\mathrm{adv}}=0.5 \cdot\left(J^{\mathrm{adv} \uparrow}+J^{\mathrm{adv} \downarrow}\right)=2 \cdot 10^{-5} \mathrm{~mol} / \mathrm{m}^{2} \mathrm{~s}$. 
The order of magnitude of diffusive and advective flux matches, and corresponds with the imposed current density. This hints that in steady state these are the dominant ion transport processes.

\subsubsection{Numerical concentration and flow calculation}

In order to gain physical insight into the nature of coupling between structure and the onset of electrokinetic instability (EKI), a theoretical framework based on Poisson-Nernst-Planck with the Navier-Stokes equations was used. Simulations of various structured membrane geometries were carried out by solving this framework numerically via the finite element method (FEM) in Comsol Multiphysics 5.2, considering a $2 \mathrm{D}$ representation of the system for numerical simplification. Quadratic Lagrange elements were used to solve for the potential and the cation/anion concentration, while the Stokes equations were solved using P2 - P1 (2nd order elements for velocity and 1st order for pressure). Mesh independence, for the approximated dimensionless Debye length, was achieved through local refinement near the membrane interface in order to resolve the regions of highest electric-field distortion. Simulations were run up to a dimensionless time of 2 (corresponding to 2500s), which was sufficient for achieving a stationary solution both in simulations and in experiments.

The dimensionless formulation of the governing equations was taken as that given previously by [25]:

Mass Conservation - Anion and Cation:

$$
\begin{gathered}
\frac{\partial c^{ \pm}}{\partial t}=-\nabla \cdot \mathbf{j}^{ \pm} \\
\mathbf{j}^{ \pm}=c^{ \pm} \mathbf{u}-\nabla c^{ \pm}-\mp c^{ \pm} \nabla \phi
\end{gathered}
$$

Navier-Stokes Equations:

$$
\begin{gathered}
\frac{1}{S c} \frac{\partial \mathbf{u}}{\partial t}=-\nabla p+\nabla^{2} \mathbf{u}-\frac{\kappa}{2 \epsilon^{2}}\left(c^{+}-c^{-}\right) \nabla \phi \\
\nabla \cdot \mathbf{u}=0
\end{gathered}
$$

Electrokinetic Dimensionless Terms:

$$
\kappa=\frac{\varepsilon_{r} \varepsilon_{0}}{\mu D}\left(\frac{k_{B} T}{z e}\right)^{2}
$$




$$
\begin{gathered}
\epsilon=\frac{\lambda_{D}}{L}, \quad \lambda_{D}=\sqrt{\frac{\varepsilon_{r} \varepsilon_{0} k_{B} T}{2(z e)^{2} c_{0}}} \\
S c=\frac{\mu}{\rho D}
\end{gathered}
$$

Poisson Equation:

$$
-2 \epsilon^{2} \nabla^{2} \phi=c^{+}-c^{-}
$$

Characteristic Scales:

$$
t_{\mathrm{diff}}=\frac{L^{2}}{D}, \quad v_{\mathrm{diff}}=\frac{D}{L}, p_{0}=\frac{\mu D}{L^{2}}, \quad V_{T}=\frac{k_{B} T}{z e}, j_{0}=\frac{D c_{0}}{L}
$$

Membrane Boundary Conditions:

$$
\mathbf{u}=0, \phi=0, c^{+}=c_{\text {mem. }}, \quad j_{y}^{-}=0
$$

Reservoir/Upper Wall Boundary Condition:

$$
\mathbf{u}=0, \quad \phi=\Delta \phi, \quad c^{+}=c^{-}=1
$$

For the potential distribution, the upper boundary was set at a constant potential while the membrane interface was considered as ground. The cation concentration at the upper boundary was set at 1 while fixed at 2 at the membrane interface, as per [25]. For anions, both upper and lower boundaries were set as no-flux, corresponding to the respective cation exchange interfaces. The fluid boundaries were no-slip at both upper and lower boundaries. The choice of the dimensionless scaling distance (relative size of Debye length to system size) was set at $10^{-3}$ for numerical stability. This choice was found previously to not strongly influence the solution $([25,30])$. The purposes of these simulations were to estimate orders of magnitude of velocity and concentration, as well as assess the coupling of the flow and electric behaviour of the system.

In the experiments the outer vortices always dominate and the flow above the gap is directed towards the membrane. In the simulations the ion transport development is calculated till a steady state occurs. Before steady state occurs near the structure corner two types of vortices are visible. On top of the structure two vortices appear with flow direction pointed to the membrane in the middle of the extrusion. These are similar to the outer vortices in the experiment. Inside the gap also two vortices are present, similar to the inner vortices in the experiments. However the inner vortices grow out of the gap, over the structure corner and become bigger than the outer vortices. In steady state the outer 
a)

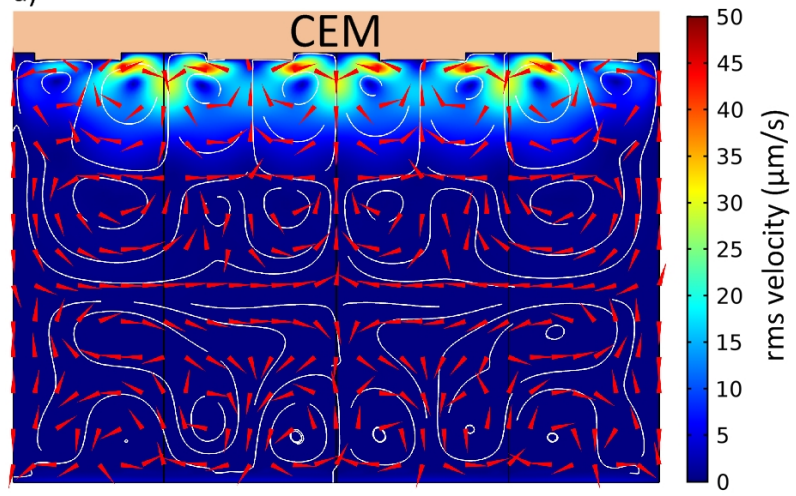

b)

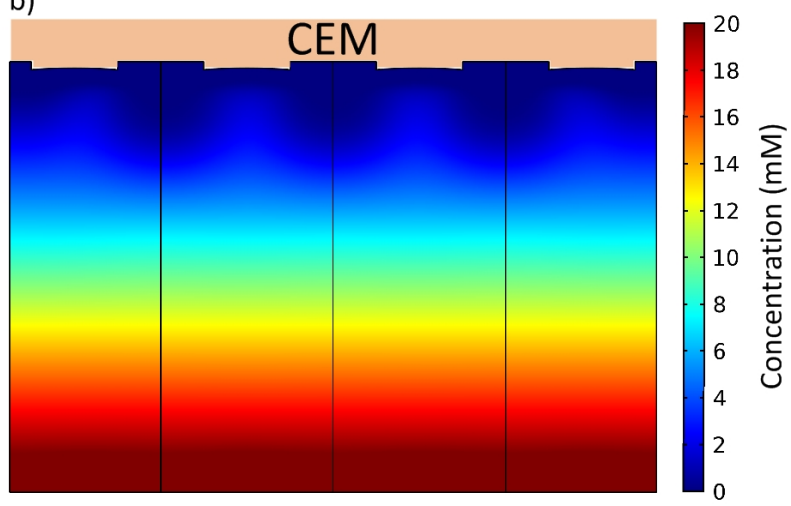

Figure 5.8: a) Numerical flow profile at a membrane with $400 \mu \mathrm{m}$ structures in $10 \mathrm{mM}$ CuSO4 with $\Delta V=0.5 \mathrm{~V}$ between the membrane interface and the opposite edge of the reservoir. b) Numerical concentration profile for a membrane with $400 \mu \mathrm{m}$ structures.

vortices disappear completely, therefore the flow direction is pointed towards the membrane at the gap and away from the membrane above the extrusion. Still the simulation results give the same order of magnitude for velocity and concentration profile as assessed by particle tracking experiments and FLIM respectively. The results for concentration are in particularly close agreement. However, as noted in the case of experimental observations the observed flow pattern (as determined via tracer particles) rotates in an opposing direction.

The precise mechanism of vortex growth likely depends on many factors, for instance the shape of the structure will influence the local electric-field profile both through changes in the local concentration profiles of anion and cations and from dielectric/conductivity contrast between the membrane and medium. 
The role of wall electroosmosis is difficult to directly assess as numerical resolution of the double layer representing the physical case $(10 \mathrm{~nm})$ is difficult for a flat membrane and the additional complication of structures only compounds the difficulty. Roughness and additional curvature effects in the membrane are also difficult to assess, as any significant geometric distortion could influence the direction and nature of the simulated EKI vortices and presumably also the experimental results. Additionally, the precise magnitude of the zeta potential at the surface is likely not fixed but varies with bulk concentration/pH effects.

Due to the uncertainty in the exact evolution of the Rayleigh number with time, see chapter 4, a fixed Rayleigh number was used based on an order of magnitude estimate for the steady-state vortex experimentally observed. This was approximately $10^{4}$, however, in reality the Rayleigh number develops dynamically. The electrohydrodynamic coupling constant in this case was approximately 0.14 for $\mathrm{CuSO}_{4}$, with a single diffusion coefficient of $0.8 \cdot 10^{-9} \mathrm{~m}^{2} / \mathrm{s}$ used to describe both anion and cation diffusivity. It is also noted that due to the nature of the electrolyte balance in the system there are potentially local $\mathrm{pH}$ gradients present and this could also influence the resulting vortex pattern, as well as have affected the tracer particle zeta potentials during particle tracking experiments [31]. The vortices simulated by Davidson et al. [18] are more similar than the ones reported here since they are sharper and directed towards the corners. Their surface consists of a (flat) rectangular pattern with patches alternating between conducting and non-conducting materials. The steady state flow is directed towards the membrane above the non-conducting patch and away from the surface at the conducting patch.

\subsection{Conclusion}

The dynamic behaviour of electroconvective vortices at structured cation exchange membranes during overlimiting current has been quantified with electrical, flow and concentration measurements. The presence of geometrical line structures on the membranes (undulations) decreases the energy needed to start and sustain electroconvection (EC). The start of EC is characterized by a sharp increase in resistance, but ends with a resistance $60 \%$ lower at the membrane with $100 \mu \mathrm{m}$ undulations compared to the flat membrane. In measurements carried out in this study, the same constant voltage drop $(1 \mathrm{~V})$ is applied. This results into vortex size growth which is accompanied by a decrease in current (increase in resistance). The system reaches a dynamic equilibrium described by a vor- 
tex mixing layer $(\mathrm{ML} \approx 0.5 \mathrm{~mm})$ and a stagnant diffusion layer $(\mathrm{DL} \approx 1.5 \mathrm{~mm})$. The current across the membrane with $400 \mu \mathrm{m}$ corrugations is the highest with a slightly thicker mixing layer. These vortices are also more stable, having less lateral motion compared to the flat membrane and therefore more efficient advective ion transport towards the membrane. Vortices seem to be stable when their height:width ration is between 1:2 and 2:1 (elongated ellipses along the surface or away from the surface). From the electrical, the flow and the concentration data, migration, advection and diffusion of ions have been quantified. Advection and migration are dominant in the ML and diffusion and migration are dominant in the DL.

These results confirm the theoretical predictions that an undulated membrane surface enhances the overlimiting current if the mixing layer height is of similar size as the undulation width. On the other hand, these results show an opposite vortex direction in contrast to what was predicted $[9,15]$. In these theoretical works a sinusoidal wave profile was taken and the flow towards the membrane is directed to the extrusion. The exact coupling mechanism at the interface remains an unanswered question. 



\section{Bibliography}

[1] S. Rubinstein, G. Manukyan, A. Staicu, I. Rubinstein, B. Zaltzman, R. Lammertink, F. Mugele, and M. Wessling, Direct Observation of a Nonequilibrium Electro-Osmotic Instability, Physical Review Letters 101, (2008). - p.114.

[2] V. Fleury, J.-N. Chazalviel, and M. Rosso, Theory and experimental evidence of electroconvection around electrochemical deposits, Phys. Rev. Lett. 68, 2492 (1992). - p.114.

[3] S. Kim, Y.-C. Wang, J. Lee, H. Jang, and J. Han, Concentration Polarization and Nonlinear Electrokinetic Flow near a Nanofluidic Channel, Physical Review Letters 99, 044501 (2007). — p.114.

[4] V. V. Nikonenko, A. V. Kovalenko, M. K. Urtenov, N. D. Pismenskaya, J. Han, P. Sistat, and G. Pourcelly, Desalination at overlimiting currents: State-of-the-art and perspectives, Desalination 342, 85 (2014). — p.114.

[5] M. Rosso, Electrodeposition from a binary electrolyte: new developments and applications, Electrochimica Acta 53, 250 (2007). — p.114.

[6] H. Strathmann, Membrane science and technology series, 1st editio ed. (Elsevier, 2004), No. 9. - p.114.

[7] N. W. Rosenberg and C. E. Tirrell, Limiting Currents in Membrane Cells, Industrial \& Engineering Chemistry 49, 780 (1957). — p.114.

[8] V. V. Nikonenko, N. D. Pismenskaya, E. I. Belova, P. Sistat, P. Huguet, G. Pourcelly, and C. Larchet, Intensive current transfer in membrane systems: Modelling, mechanisms and application in electrodialysis, Advances in Colloid and Interface Science 160, 101 (2010). - p.114, 126.

[9] I. Rubinstein and B. Zaltzman, Electro-osmotically induced convection at a permselective membrane, Physical Review E 62, 2238 (2000). — p.114, $119,121,131$.

[10] M. Z. Bazant, M. S. Kilic, B. D. Storey, and A. Ajdari, Towards an understanding of induced-charge electrokinetics at large applied voltages in concentrated solutions., Advances in colloid and interface science 152, 48 (2009). - p.114.

[11] S. S. Dukhin, Electrokinetic phenomena of the second kind and their applications, Advances in Colloid and Interface Science 35, 173 (1991). — p.114. 
[12] N. a. Mishchuk, Concentration polarization of interface and non-linear electrokinetic phenomena., Advances in colloid and interface science 160, 16 (2010). - p.114.

[13] I. Rubinstein and B. Zaltzman, Electro-convective versus electroosmotic instability in concentration polarization., Advances in colloid and interface science 134-135, 190 (2007). - p.114.

[14] I. Rubinstein and B. Zaltzman, Dynamics of extended space charge in concentration polarization, Physical Review E 81, 061502 (2010). — p.114, 126.

[15] E. a. Demekhin, N. V. Nikitin, and V. S. Shelistov, Direct numerical simulation of electrokinetic instability and transition to chaotic motion, Physics of Fluids 25, 122001 (2013). — p.114, 121, 131.

[16] E. Karatay, C. L. Druzgalski, and A. Mani, Simulation of chaotic electrokinetic transport: Performance of commercial software versus custom-built direct numerical simulation codes, Journal of Colloid and Interface Science 446, 67 (2015). - p.114.

[17] H. C. Chang, E. A. Demekhin, and V. S. Shelistov, Competition between Dukhin's and Rubinstein's electrokinetic modes, Physical Review E 86 , (2012). - p.114.

[18] S. M. Davidson, M. Wessling, and A. Mani, On the Dynamical Regimes of Pattern-Accelerated Electroconvection, Scientific Reports 6, 1 (2016). p.114, 122, 123, 130.

[19] J. Balster, M. H. Yildirim, D. F. Stamatialis, R. Ibanez, R. G. H. Lammertink, V. Jordan, and M. Wessling, Morphology and microtopology of cation-exchange polymers and the origin of the overlimiting current., The journal of physical chemistry. B 111, 2152 (2007). - p.115, 116, 119.

[20] M. Wessling, L. G. Morcillo, and S. Abdu, Nanometer-thick lateral polyelectrolyte micropatterns induce macrosopic electro-osmotic chaotic fluid instabilities., Scientific reports 4, 4294 (2014). - p.115.

[21] N. D. Pismenskaya, V. V. Nikonenko, N. A. Melnik, K. A. Shevtsova, E. I. Belova, G. Pourcelly, D. Cot, L. Dammak, and C. Larchet, Evolution with time of hydrophobicity and microrelief of a cation-exchange membrane surface and its impact on overlimiting mass transfer, , The journal of physical chemistry. B 116, 2145 (2012). — p.115.

[22] E. Korzhova, N. Pismenskaya, D. Lopatin, O. Baranov, L. Dammak, and V. Nikonenko, Effect of surface hydrophobization on chronopotentiometric behavior of an AMX anion-exchange membrane at overlimiting currents, Journal of Membrane Science 500, 161 (2016). — p.115.

[23] D. E. Lide, CRC Handbook of Chemistry and Physics, 76th ed. (CRC Press, 1996), pp. 5-90. - p.116, 126. 
[24] J. C. de Valença, R. M. Wagterveld, R. G. H. Lammertink, and P. A. Tsai, Dynamics of microvortices induced by ion concentration polarization, Physical Review E 92, 031003 (2015). — p.120.

[25] C. L. Druzgalski, M. B. Andersen, and a. Mani, Direct numerical simulation of electroconvective instability and hydrodynamic chaos near an ion-selective surface, Physics of Fluids 25, 110804 (2013). - p.120, 126, 127, 128.

[26] S.-J. Liu, H.-H. Wei, S.-H. Hwang, and H.-C. Chang, Dynamic particle trapping, release, and sorting by microvortices on a substrate, Physical Review E 82, 026308 (2010). - p.122.

[27] Y. Green and G. Yossifon, Dynamical trapping of colloids at the stagnation points of electro-osmotic vortices of the second kind, Physical Review E 87, 033005 (2013). - p.122.

[28] A. Yaroshchuk, E. E. L. Bernal, and T. Luxbacher, Electrokinetics in undeveloped flows, Journal of Colloid and Interface Science 410, 195 (2013). - p.125.

[29] E. A. Demekhin, S. Amiroudine, G. S. Ganchenko, and N. Y. Khasmatulina, Thermoelectroconvection near charge-selective surfaces, Physical Review E Statistical, Nonlinear, and Soft Matter Physics 91, 1 (2015). — p.126.

[30] C. Druzgalski and A. Mani, Statistical analysis of electroconvection near an ion-selective membrane in the highly chaotic regime, Physical Review Fluids 1, 073601 (2016). - p.126, 128.

[31] R. Femmer, A. Mani, and M. Wessling, Ion transport through electrolyte/polyelectrolyte multi-layers., Scientific reports 5, 11583 (2015). p.130. 



\section{CHAPTER 6}

\section{Summary and outlook}

\subsection{Summary}

The work presented in this thesis is concerned with the understanding and exploitation of overlimiting current (OLC) phenomena in the vicinity of ion exchange membranes. OLC is a boundary phenomena in which additional ion transport next to ion migration and diffusion starts, thereby increasing the current through the membrane. This work has focused on advection driven OLC at cation exchange membranes (CEM), since at a CEM water dissociation during OLC is negligible. Advection, the transfer of heat or matter by the flow of a fluid, was induced at the membrane in two ways. Firstly, by direct coupling of the electric field and the ions in the charged layer at the membrane interface. Since there is a net charge a net ion flow occurs at the interface, the liquid is set in motion under sufficient electrical forcing. This phenomena is called electroconvection, where convection refers to the sum of advective and diffusive transport. Secondly, the electric field also induces a concentration gradient due to the selectivity of the membrane. This fact induces a density gradient that can become gravitational unstable due to buoyancy forces, giving the second type of convection, called gravitational convection. This occurs only if the lighter (ion depleted) layer lies below the heavier (ion enriched) layer which is referred to as the gravitational unstable orientation.

Both types of convection (electro- and gravitational) are induced in a transparent electrochemical cell in which the fluid is initially stagnant. The timedependent electrical resistance over the membrane is measured while keeping either the current or voltage drop fixed. Simultaneously the emerging fluid motion is monitored by recording tracer particles suspended in the fluid. The particle images are analysed by following the single particle motion and by using particle image velocimetry (PIV) algorithms to determine the collective particle motion. Particle tracking allows an accurate determination regarding advection onset, growth and magnitude. Using another optical technique called fluorescence lifetime image microscopy (FLIM), the concentration distribution was extracted. 
The combination of above techniques makes is possible to determine the ion transport via diffusion, migration and advection and compare this to theoretical and numerical predictions (Chapter 1 and 2)

The electroconvective mixing layer near a flat cation exchange membrane starts quickly after the interface gets depleted and an additional threshold voltage is applied. The interface layer becomes unstable which starts a vortical flow which in turn enhances the instability. This positive feedback loop is referred to as the electrokinetic instability (EKI). The PIV data shows a layer of lateral moving vortices with approximately equal height and width. The height of the layer grows, while the number of vortices shrink. Although the mixing layer grows till an approximately fixed saturated thickness, the individual vortices keep moving chaotically along the membrane. Applying a higher current density results in a thicker mixing layer and higher system resistance. The conductivity of the mixing layer was found to be nearly constant in all experiments (Chapter 3). This has led to the hypothesis that the mixing layer has a fixed average concentration, independent of size, while the stagnant layer has a concentration gradient. This hypothesis was supported by measuring the concentration profile within the system with FLIM (Chapter 4).

The development of the concentration profile within the system domain is also predicted with a numerical model based on Fick's laws of diffusion with constant flux boundary conditions in a stagnant liquid. The model predictions agree with the measured concentration profile development and predict the time the interface gets depleted, at which point electroconvection starts. The theoretical depletion time agrees with the electrical transition time and electroconvection onset time observed in the gravitational stable orientation where no gravitational convection can occur (Chapter 3 and 4). This orientation is also called counter-orientation, since the electrical field and the gravitational field point in opposite directions. In co-orientation both fields point downward and the model is also used to predict when the concentration gradient becomes gravitationally unstable and Rayleigh-Bénard (RB) convection occurs. To test this, the cell is operated in two orientations towards the gravitational field and shows that the numerical and experimental transition times are in agreement. The measurements also show that RB convection occurs in the full cell, therefore reducing the resistance. At high current electroconvection via the electrokinetic instability (EKI) and RB coexist, since the additional ion transport via RB is not enough to avoid the interface layer gets fully depleted. The RB motion does reduce the diffusion boundary layer, therefore diminishing the EKI layer thickness drastically (Chapter 4). This is similar to the reduction of the diffusion boundary layer because of pressure driven flow.

The exact coupling between the electric field and the EKI mixing layer is 
a much debated question. To understand the onset of EKI, membranes with different line undulations are compared under constant potential conditions. The presence of the structures reduces the threshold voltage to start electroconvection up to $60 \%$ compared to at a flat membrane (Chapter 5). The structures act as starting points for electroconvection which results in periodic vortices, compared to a flat membrane where the vortices appear at random locations and move lateral along the surface. The structured membrane interface has a part that is parallel to the external electric field, along which an electro-osmotic flow (wall EOF) occurs. Our hypothesis is that wall EOF occurs without a threshold and increases in strength as the concentration depletes further and the space charge layer expands. This leads to periodic heterogeneity in the concentration profile and electric field that allows EKI to start at a lower threshold voltage.

After the onset the vortices grow until saturating in size. When the membrane structures had similar dimension as the saturated mixing layer thickness, the resistance of this layer was $50 \%$ lower compared to a flat membrane. The surface structures fix the vortices position allowing steady inflow locations with higher concentration and outflow locations with lower concentration. The PIV and FLIM results show the mixing occurs in vortex rolls along the line structures (Chapter 5). With the same driving force the vortices can grow larger, therefore extend the mixing layer farther from the membrane interface and bring higher concentration solution to the membrane. This results in improved average concentration in the mixing layer and a reduced resistance. From the resistance, concentration and flow data the migration, diffusion and advective transport is estimated. These calculations give the right order of magnitude of ion transport. This hints that in steady state no other major transport processes are significant (e.g. transport by other ions $\left(\mathrm{H}^{+}, \mathrm{OH}^{-}\right)$or loss of ions due to chemical reactions (copper oxide scaling)).

To summarize, the work presented in this thesis provides the observation and determination of ion concentration polarization (ICP) by applying techniques such as fluorescence lifetime image microscopy (FLIM), particle tracking and particle image velocimetry (PIV). This latter technique was also used for proving that electroconvection is a source of overlimiting current (OLC) regime at cation exchange membranes. A new regime of chaotic motion in the electroconvective mixing layer has been observed in contrast of what was previously described by other researchers. Another new aspect of this work is the observation and classification of the coupling between electroconvection and Rayleigh-Bénard convection. Furthermore, the work presented in this thesis quantifies the behaviour of the electroconvective vortices at geometrically structured membranes. When the mixing layer thickness and the structure periodicity have a similar length the vortices are stabilised which reduces the resistance within the layer. 


\subsection{Outlook}

While the previous section (summary) describes the insights provided by this research, this section (outlook) focuses on the (new) questions and ideas this research has generated. To understand the new questions one has to understand the scope of the project, which was part fundamental (how does OLC work?) and part practical (how can OLC be applied?). Fundamentally the project aimed to investigate overlimiting current with higher experimental detail then previously done [1] and narrow the gap between the theoretical predicted [2] and experimental reported [3] reduction of OLC resistance at structured membranes. Only electrical resistance data was reported and with improved optical techniques (PIV and FLIM) more insight in the interfacial phenomena could be obtained. Practically the project aimed to find design principles for membranes with enhanced electroconvection.

An improvement for enhanced electroconvection has been found when the membrane has periodic structures with the same size as the mixing layer thickness. This promotes stable vortices with a lower electrical resistance. However, the exact nature of the electroconvective coupling remained out of the scope of the experiments, since the coupling occurs in the $0.1 \mu \mathrm{m}$ thick interface layer, and the rotation of the experimentally observed vortices are opposite of what was predicted and numerically simulated (Chapter 5). A major open fundamental question that arises is: "What determines the initial coupling for the directionality of the vortices?". The practical questions ("How can we use this result?") come along with every finding.

The next subsections describe these fundamental and practical questions and provide ideas on how to answer them. First, the question and ideas relation to membrane shape optimization are discussed. Next to different geometrical structures, also chemical structured membranes are discussed. Second, the comparison between experimental and numerical work is discussed. Both experimental and numerical research approaches can give unique insight in the question regarding overlimiting current. Observations of both communities match qualitatively, but not quantitative yet. Several approaches are given on how to get quantitative agreement. Third, an operational system is presented in which the the electroconvection reduces the resistance to near Ohmic values. This occurs in small systems, where the electroconvective vortices reach the other side and give similar mixing as Rayleigh-Bénard convection does. And fourth, some ideas on applications with overlimiting current are given. Two properties of OLC are discussed, the enhanced current density vs. the limiting current density and the fluid mixing. 


\subsubsection{Membrane shape optimization}

The current hypothesis is that the electroconvection is driven by collective motion in the extended space charge layer, $\mathrm{a} \sim 100 \mathrm{~nm}$ thick layer that forms if the interface concentration get depleted and an excess voltage is applied. It is therefore an out-of-equilibrium phenomena. At ideally flat membranes this layer extends until a spacial inhomogeneity triggers an electroconvective vortex which in turn enhances the inhomogeneity. This mechanism is known as the RubinsteinZaltsman mechanism, also called the electro-kinetic instability. When the membrane is curved the surface has a component parallel to the electric field, along which electro-osmotic flow can occur. This flow is stronger when the electric space charge layer (ESCL) expands. This phenomena is known as the Dukhin mechanism, or electro-osmosis of the second kind. At structured membranes both mechanisms (Dukhin and Rubinstein) can take place. The surface curvature can enhance the onset and stabilize the flow direction, therefore enforcing instability feedback mechanism.

By varying the surface shape it is possible to better understand how the two mechanisms interact and what the optimal membrane shape is to decrease the resistance of the mixing layer. To do so parameters like the height of the pattern, the corner angle and the gap/extrusion ratio can be investigated to find the optimal structures, see figure 6.1. The corner rounding is expected to have a significant impact on the electric field distribution, therefore the electro-osmosis coupling. At round corners the electro-osmotic flow (Dukhin) is expected from extrusion to bottom along the whole width. The flow towards the membrane will be directed to the extrusion and away from the gap. When the structure height of rectangular vortices is increased (figure 6.1-top-right) the electrolyte in the gaps will become depleted while being stagnant, being virtually non-conductive. The observed vortices will be similar vortices as numerically prediction on a flat membrane with conducting and non-conducting patches [4].

Structured membranes can be made by different techniques. Membranes in Chapter 5 were made by casting a polymer solution on a micro-structured mold, see figure 5.1, made by photolithography and etching [3]. This technique has the advantage of being really precise, but also requires a specialized facility. Moreover, the pattern can only be produced with one depth at the time. Since $0.1 \mathrm{~mm}$ is relatively large other techniques can be applied to create the mold, e.g. 3D printing. It might also be possible to directly print the membrane itself [5] or to modify an existing membrane by hot pressing [6].

Not only geometrical structures, but also chemical heterogeneities on an ion exchange membrane can influence interfacial ion concentration and electric field distribution, thereby influence electroconvection. A benefit of chemical pattern- 


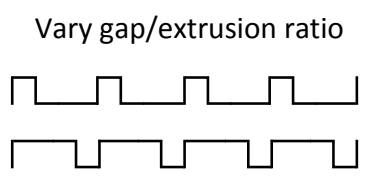

Vary corner angle

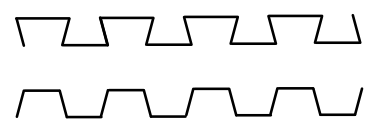

\section{Vary structure height}
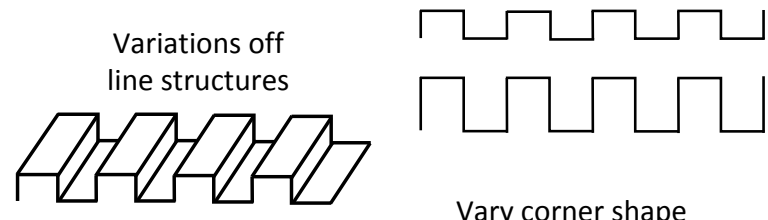

Vary corner shape
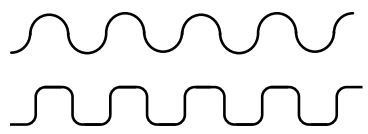

Figure 6.1: In chapter 5 rectangular line structures have been investigated (middle figure). The corner of the structure can influence the electroconvection (bottom left and right. Also the structure height and gap/extrusion ration are interesting parameters to study regarding their influence on the stability of electroconvective micro-vortices.)

ing is that an existing membrane can be modified with a thin chemical layer. Research showed that membranes with patterned chemical layers have an enhanced onset of overlimiting current (OLC) [7]. Cation exchange membranes were coated with a hexagonal dot pattern from polyelectrolyte with positive charged poly(allylamine hydrochloride) ( $\mathrm{PAH})$ and negative charged poly(acrylic acid) (PAA) $[7,8]$. Some of these membranes were investigated in the copper electrode membrane cell filled with a $\mathrm{CuSO}_{4}$ electrolyte, but the observations of [7] could not be reproduced. A copper oxide layer deposited at the depleted side of the membrane, most probably due to catalytic reactions at the anion exchange PAH layer. Water dissociation at anion exchange membranes is known to occur under overlimiting current conditions $[9,10]$. $\mathrm{A} \mathrm{CuSO}_{4}$ electrolyte solution is not stable as the $\mathrm{pH}$ increases [11]. The reported enhancement was seen in a $\mathrm{NaCl}$ solution. By filling the copper electrode cell with $\mathrm{NaCl}$ solution the enhanced onset of these chemically patterned membranes might be reproduced.

Next to chemical patterning of the surface charge, also patterning of conductivity [4] or hydrophobicity [12] can result in periodic interface concentration and electric field, therefore enhance the onset and stability of electroconvection. Such a membrane can be made by micro contact-printing the desired top layer or by electrospraying or electrosputtering of the desired top layer on a partly covered membrane. Patterned conductive membranes might also be made by casting a membrane polymer between a grid of non-conductive material. Actually to enhance the electroconvective properties the whole membrane is desired to be hydrophobic since this decreases the solid-liquid friction, therefore enhancing the electro-osmotic momentum transfer [12]. 


\subsubsection{Comparison between numerical and experimental work}

The core of science is to understand the working of the natural world. The scientific method consists of predicting what will happen under certain conditions and testing if the predictions are correct and reconsidering the first prediction. Numerical methods can be seen as an extension of the theoretical prediction, based on the fundamental physical and chemical principles underlying the process at hand. Experimental methods are used to test these predictions and judge the proposed theory.

The advances on computational calculation power opened the door to resolve theoretical predictions in high resolution, with precision. Recent advances in direct numerical simulations (DNS) have successfully resolved the Poisson-NernstPlanck (PNP) equations together with the Navier-Stokes (NS) equations from first principle using finite element methods. The DNS data contains the timedependent values of all variables, i.e. concentration distribution, flow field, electrical field, local pressure. This gives a deep understanding of what is happening, and detailed predictions. The direct numerical calculations describing the onset and growth of electroconvection are computational costly, since the grid size needs to be fine enough to resolve the fluctuations at the thin, $\mathcal{O}\left(\lambda_{D} \sim \mathrm{nm}\right)$, electric double layer that is essential to the process. For this simplifications are made for which only experimental observations can determine if they are valid.

\section{Simulations approach experimental observations}

At this moment the numerical simulations in a depletion layer with a domain size $1000 \times$ bigger than the Debye length have been resolved, $\epsilon=\lambda_{D} / L=10^{-3}$, $2 \mathrm{D}[4,13-15]$ and recently also in $3 \mathrm{D}[16,17]$. This can be converted to an experimental system with $10 \mathrm{mM} \mathrm{CuSO}_{4}\left(\lambda_{D} \approx 2 \mathrm{~nm}\right)$ with a height of $2 \mu \mathrm{m}$. The numerical currents and velocities do not directly align with the experimental values. Increasing the numerical resolution is a way to get a valid comparison. Simulations done with $\epsilon=10^{-4}$ showed the qualitative behaviour does not change significantly. Also 3D simulations at flat membranes did show qualitative the same behaviour, but not quantitative [17]. For better comparison high resolution and 3D numerical solutions are needed with qualitative predictions on system resistance and resulting the vortex structures, e.g. vortex rolls or hexagonal structures [16].

Another way to improve the comparison between experiment and numerics is to simulate the tracers used in experiments. An example is the simulations of tracer particles with a small zeta potential in an electroconvective flow by Davidson et. al. [4]. They showed the formation of particle free areas (voids), similar to the experimental results in Chapter 3, see figure 6.2. The particles were 
ideal tracers, under influence of a simple electrophoretic force, eq. 2.6. Other effects that could cause a difference between particle motion and flow motion (e.g. stokes drag, gravitational settling, dielectrophoresis, see section 2.2.2) were neglected. Numerical simulations can be improved further by including such interactions.

For simplification the numerical system is often assumed to consist of a binary strong electrolyte, meaning only two opposite charged ions are present that are fully dissociated. In real systems chemical and electrochemical (dissociation) reactions take place. Numerical approaches have been designed to take this into account in $1 \mathrm{D}$ [18]. Combining 2 or 3D electroconvection simulations with chemical equilibrium calculations can predict $\mathrm{pH}$ gradients and the formation of scaling, like the copper oxide layer at the PAH coated membranes in the previous section. Once pH gradients are known, one might even be able to calculate the light efficiency of fluorescence tracer molecules that depend on $\mathrm{pH}$ or ionic strength.
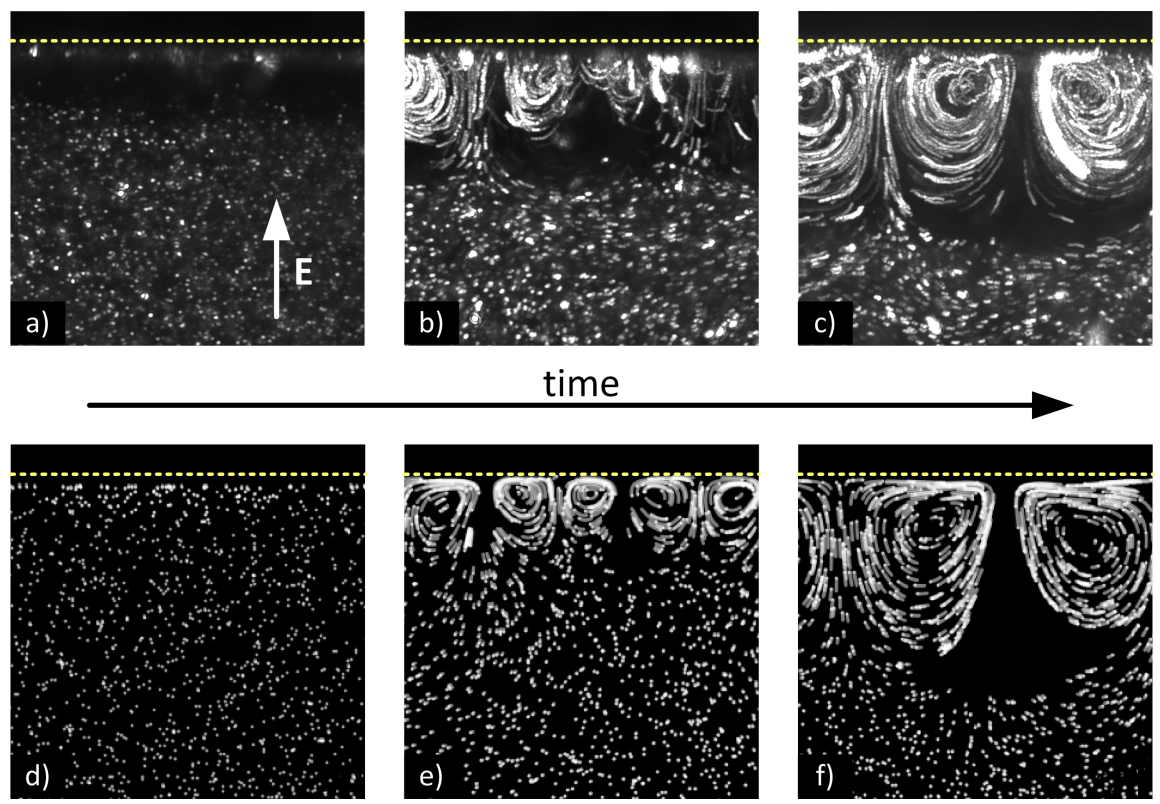

Figure 6.2: (d-f) Numerical prediction of the pathlines of tracer particle with a zetapotential of $-1 \mathrm{mV}$. Simulations are preformed to match the experimental observation (a-c) described in Chapter 3. Numerical results come with courtesy of Scott Davidson, see [4] for details on the simulations. 


\section{Experiments approach numerical simulations}

To check high resolution predictions, high resolution experiments are needed. This thesis shows a way to measure a quantitative concentration field next to a membrane with fluorescence lifetime image microscopy (FLIM). The temporal resolution $(\sim 5 \mathrm{~s})$ is too slow to see the fast lateral motion along the membrane and the spatial resolution too low to see inhomogeneities in ion concentration very near the membrane surface that have been seen numerically, see figure $6.3 \mathrm{~b}$. Image techniques based on fluorescence light intensity use all emitted light, therefore images can have a higher temporal and spatial resolution. Although movies of the fluorescent light intensity are challenging to connect to actual ion concentration, they did show the fast and small concentration inhomogeneities near the membrane, see figure 6.3a.

The image in figure $6.3 \mathrm{a}$ is made with the fluorescent setup described in figure 2.10 and represents the average light intensity over $0.9 \mathrm{~s}$. The cell was filled with $27 \mathrm{mM} \mathrm{NaCl}$ and $10 \mu \mathrm{M}$ negatively charged Alexa 488 dye and the voltage was increased in time $(0-2 \mathrm{~V}, 3 \mathrm{mV} / \mathrm{s})$. In $\mathrm{NaCl}$ the Alexa light intensity is known to follow the concentration profile [20]. The $\mathrm{pH}$ and transparency of $\mathrm{NaCl}$ do not depend on ion concentration, in contrast to $\mathrm{CuSO}_{4}$, see section 2.1.1. The copper electrode reactions bring $\mathrm{Cu}^{2+}$ into the electrolyte which complicates the chemistry of the system in time. It was not possible to relate the light intensity to concentration, but the dynamics show fast moving small vortices at the membrane and larger and steady mushroom shaped vortices pointing out, see figure 6.3a. This is similar to the numerical calculated concentration distribution although
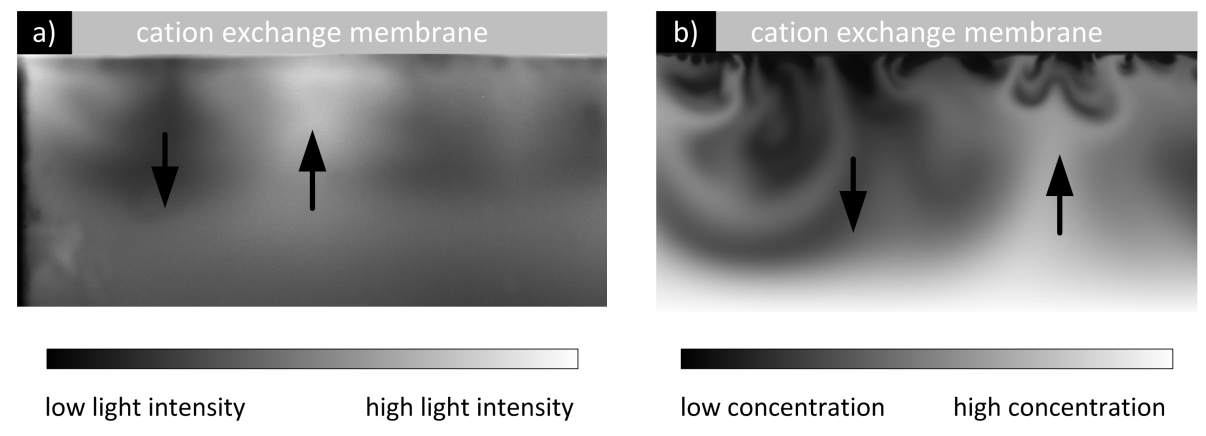

Figure 6.3: a) Experimental snapshot of the Alexa dye light intensity influenced by the electroconvection vortices. b) A simulation of the ion concentration inside the electroconvective vortices. The colour is proportional to the cube-root of concentration. The simulation is done with $\epsilon=10^{-4}$, see [13, 19] for more information. Image with courtesy of Ali Mani as personal communication. 
it represents a smaller scale, figure 6.3b. A statistical analysis on frequency of the interface fluctuations could be a way to compare the visualization with the numerical results can be achieved. This can give insight why these phenomena on different scale look similar and how they differ.

The experimental images presented represent a cross-section of the three dimensional phenomena. Two counter-rotating vortices can be a cross-section of two vortex rolls or a toroidal (donut) vortex [16]. With the investigation of line structures vortex rolls are generated, while at the flat membrane toroidal vortices exist, as the flow data suggests. To really see the three dimensional structure in the flow the easiest step is to make slices at different positions on the $\mathrm{z}$ axis. This can be done by moving the system or by confocal microscopy [21, 22]. More complicated techniques to see the particle motion along the $z$ axis are by using a camera with a cylindrical lens, astigmatism particle tracking velocimetry (APTV) [22], or stereographic PIV of tomographic PIV [23, 24]. With a setup capable of seeing three dimensional flow patterns it is possible to investigate the flow at the front and back end of the vortex roll. The walls will most probably have an effect on the vortex confinement and therefore stability. Also 3D visualizations open up the possibility to investigate more complex patterns, like hexagonal structures $[7,16]$. Looking directly on top of the membrane surface might reveal the dynamics of the onset of electroconvection by visualizing the depleted areas [17]. When the membrane is transparent the interface depletion can be seen from the back with a fluorescent dye and total internal reflection fluorescence (TIRF) [25]. Another way is to use induce ion concentration polarization with resulting electroconvection at a transparent electrode, e.g. indium-tin-oxide (ITO) [26].

The last experimental challenge to discuss here is the multi-valued nature of the overlimiting resistance. When experiments (e.g. with constant applied voltage difference as in Chapter 5) are repeated the resulting resistance varies. This is normally attributed to small experimental deviations such as in temperature, concentration, pressure, cell and membrane orientation, electrode surface and most of the times due to leaking of water (the nightmare of every water experimentalist). Variations in initial resistance can be in the order of $0.5 \mathrm{k} \Omega$, while the variations in the resulting saturated overlimiting resistance can be in the order of $7 \mathrm{k} \Omega$ (in the case of the chronoamperometry experiments in Chapter 5). In a recent numerical paper of Davidson et al. [4] the authors showed that also small random numerical initial perturbations can lead to different steady state vortices. If vortices become big, the instability feedback mechanism keeps them big, which results in effective advective ion transport towards the membrane surface. If the vortices are initially small, they stay small which results in less effective advection and a higher resistance compared to the bigger vortices. 
Whether the vortices are small or big depend on the initial inhomogeneities in the extended space charge layer which triggers the electrokinetic instability. Also do big vortices seem to attract big vortices and small vortices attract small ones. This suggests that the experimental reproduction errorbars are due to unavoidable fluctuations, e.g. thermal fluctuations or a micrometer shift in membrane placement. The errorbars in figure $5.3 \mathrm{c}$ can be a result of this multi-valued overlimiting current behaviour. Detailed experimental studies on the reproducibility will most likely show that when the resulting resistance is lower the vortices are bigger, while when the resistance is higher the vortices are smaller.

\subsubsection{Reduced resistance due to electroconvection}

In the copper electrode cell a situation was found where the electroconvection mixed the complete compartment, bringing the resistance down to near ohmic resistance of the unmixed homogeneous $\mathrm{CuSO}_{4}$ layer. This mixing occurred in a set of experiments where the anode compartment height was varied. Decreasing the compartment height from $2 \mathrm{~mm}$ to $0.5 \mathrm{~mm}$ showed that the vortices reach all the way to the anode, see figure $6.5 \mathrm{~h}$. These vortices therefore bring the high concentrated anode boundary fluid faster than diffusion to the membrane.
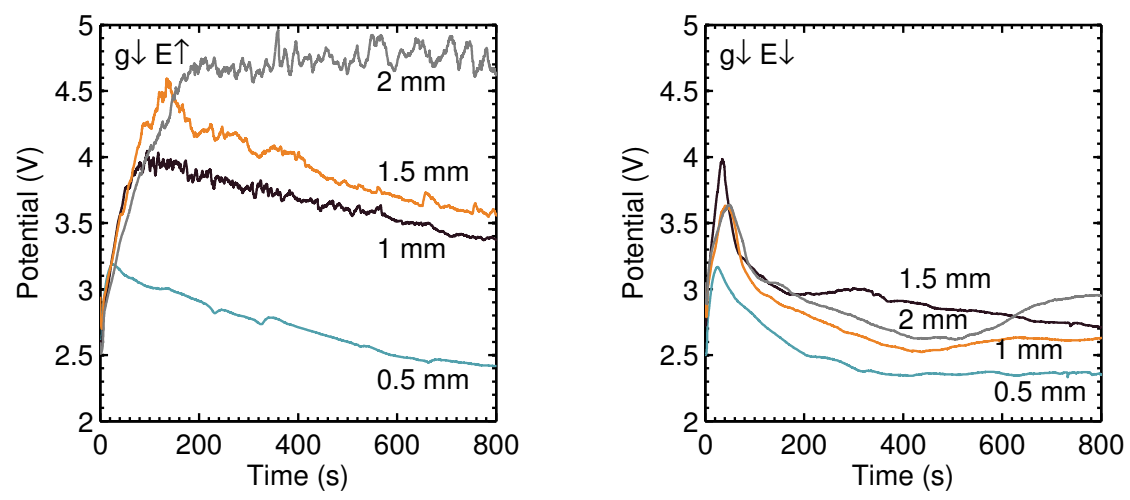

Figure 6.4: A constant current $\left(4.4 \mathrm{~A} / \mathrm{m}^{2}\right)$ is forced through compartments of different height filled with a $1 \mathrm{mM} \mathrm{CuSO}_{4}$ solution. The transition time is the same for all sizes, $\tau_{\mathrm{s}} \approx 4 \mathrm{~s}$. The voltage is measured between anode and reference electrode $(7.3 \mathrm{~mm}$ away from membrane). The initial voltage difference is small $(\Delta V=0.4 \mathrm{~V}$ for thin and thick reservoir. The limiting current in the thick $(2 \mathrm{~mm})$ reservoir is $16 \cdot i_{\text {lim }}$ and in the thin $(0.5 \mathrm{~mm})$ reservoir $4 \cdot i_{\text {lim }}$. Left shows the gravitational stable, counter-orientation and right the gravitational unstable, co-orientation. 
co-orientation: $\mathrm{E} \downarrow \mathbf{g} \downarrow$

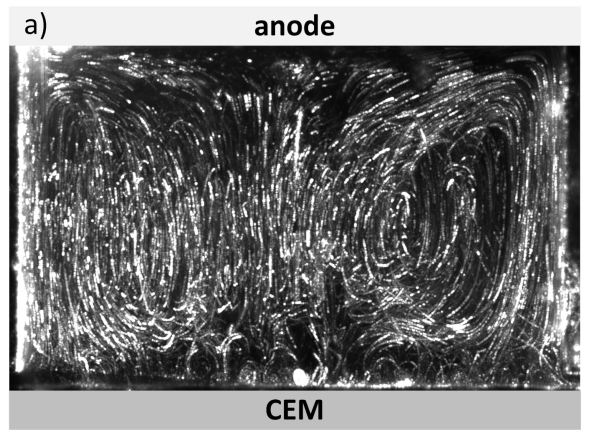

b)

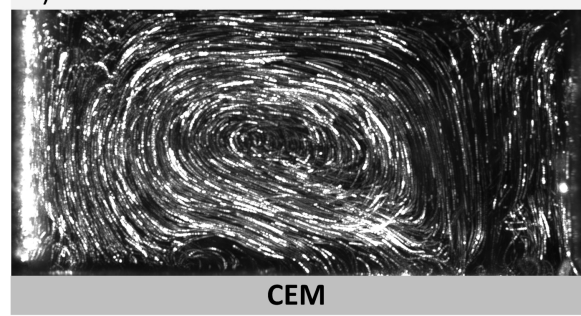

c)

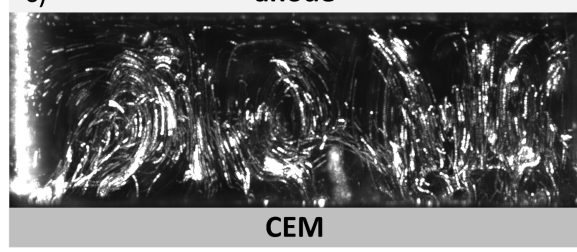

d)

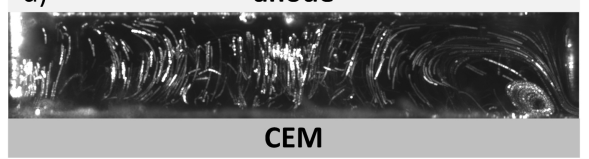

counter-orientation: $\mathrm{E} \uparrow \mathbf{g} \downarrow$
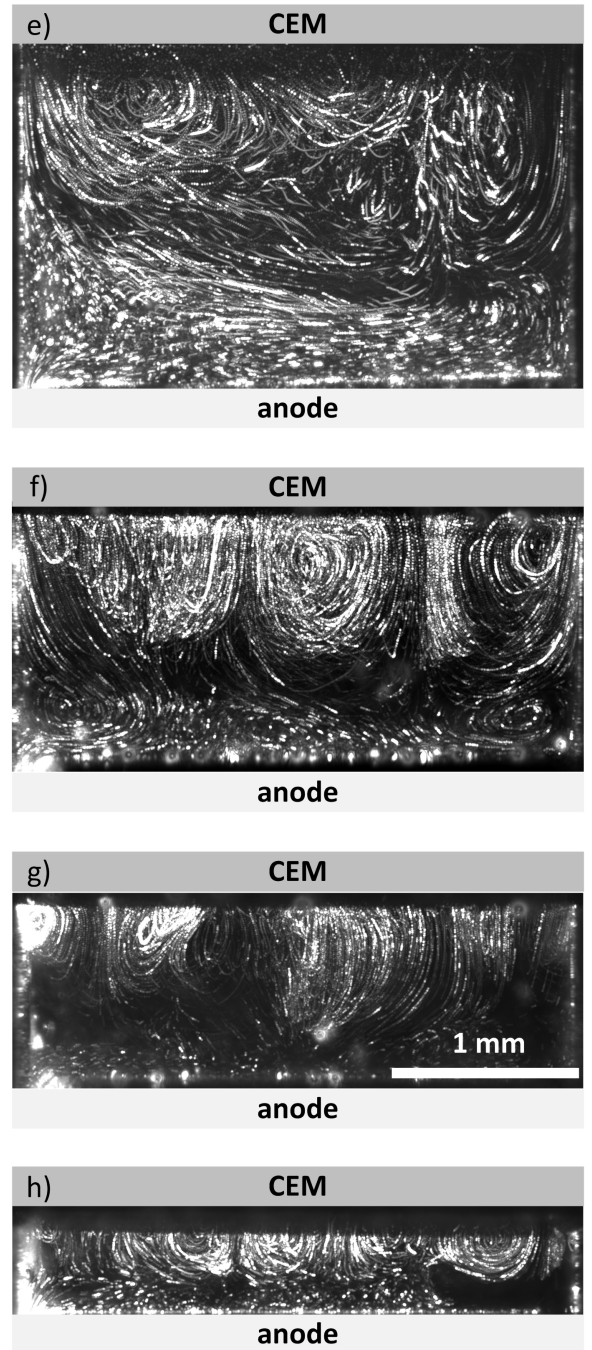

Figure 6.5: Images of particle pathlines $(\Delta t=10 \mathrm{~s})$ during overlimiting current in the copper electrode cell with compartment size with $1 \mathrm{mM} \mathrm{CuSO}_{4}$ while applying 4.4 $\mathrm{A} / \mathrm{m}^{2}$ at $\mathrm{t}=250-260 \mathrm{~s}$. In the co-orientation (a-d) RB convection occurs in the full cell and EKI at the membrane. In the counter-orientation (e-h) a highly chaotic EKI mixing layer occupies more than half of the cell. In figure $(\mathrm{h})$ the mixing brings the enriched fluid near the anode to the membrane. Compartment height decreases with steps of 0.5 $\mathrm{mm}$. 
First the resistance shoots up after the transition time due to the depleted highly resistive layer, see figure 6.4. When the voltage is increased the vortices grow and the system separates into a mixing layer with low ion concentration and high resistance and a diffusion layer with higher ion concentration and lower resistance. In the co-orientation (figures 6.4-right and 6.5a-d) Rayleigh-Bénard convection quickly starts mixing the whole cell and decreasing the resistance to near ohmic values. In the counter orientation (figures 6.4-left and 6.5e-h) the electroconvective mixing layer (that mixes via the electrokinetic instability, EKI) grows till $\approx 1 \mathrm{~mm}$. In the smallest compartment EKI mixes the whole cell and decreases the resistance to near ohmic values.

This special situation occurs due to the special geometry of the cell, where the solution is confined between two cation selective surfaces, keeping the average anion concentration fixed. The cell mimics a stagnant layer, but with fixed average concentration. The more the membrane side is depleted the higher the anode concentration becomes. The bigger the vortices become relative to the stagnant layer, the more efficient does the mixing become. There is no sharp transition between mixing layer and diffusion layer, since the mixing always generates secondary vortices. At higher voltages this secondary mixing becomes more pronounced. The electroconvective mixing reaches further and therefore brings higher concentrated fluid down. This increases the average concentration in the mixing layer thus lowers the resistance.

The snapshot of the motion in the co-orientation (figure 6.5a-c) show small vortices near the CEM that reveal the EKI mixing layer. At the small compartment it is unsure if Rayleigh-Bénard (RB) convection is present. The limiting current gradient $(\Delta c=2 \mathrm{mM}, L=0.5 \mathrm{~mm})$ gives a Rayleigh number of $R a=450$, see eq. 4.7. With decreasing compartment size RB convection diminishes and EKI mixing may be a better way to mix the full compartment.

\subsubsection{Final remarks on applications with overlimiting current}

The most obvious application of overlimiting current is to use the high current density to intensify processes. In electrodialysis (ED) electroconvection can enhance the current, thus the desalination rate. However the resistance increases (see figure 2.7-right) and the energy efficiency decreases by more than half compared to the underlimiting efficiency, see figure 2.4-right. When the capital investment outweighs the operational costs it can be a choice to operate an electrodialysis system in overlimiting current regime as calculated by Nikonenko et al. [27]. Fewer membrane stacks will be needed that operate in the more energy expensive overlimiting current mode to have the same product results. The use of membranes with enhanced overlimiting current properties (as shown in Chapter 
5) will be beneficial once the overlimiting current regime is consciously chosen as a mode of operation.

Another reason the OLC regime is avoided is the start of water dissociation at the anion exchange membranes, leading to changes in $\mathrm{pH}$ in concentrated and enriched solution $[9,28]$. When the interface concentration depletes the local electric potential drop increases above a certain threshold such that catalytic reactions between the membrane surface groups and the water start. If the membranes are designed to have electroconvection with a lower threshold than the catalytic water dissociation reactions, these reactions can be avoided such that the $\mathrm{pH}$ of the solution will not change while operating in the overlimiting current regime. The other way around, if $\mathrm{pH}$ changes are desired (e.g. acid and/or base production processes $[29,30]$ ) the membranes can be tuned to have electroconvection occur at a higher threshold than water dissociation (e.g. flat and hydrophilic membranes).

The orientation of the concentration gradients towards the gravitational field can be used to enhance or reduce gravitational convection. Due to the geometry of the experimental system (initially stagnant layer between two cation selective surfaces) the gravitational convection mixes the whole cell reducing the electrolyte resistance to near ohmic values. In an ED compartment the gradient at the CEM is opposite to the AEM. In a horizontal orientation one could become unstable, while the other is stable. In a vertical orientation both gradients become unstable. In case of a pressure driven (Poiseuille) flow in the boundary the occurrence of gravitational convection complicates, but is still present [31, 32]. A vertical orientation of the membrane stack is therefore preferred to (when possible) induce the (free) gravitational convection to enhance the ion transport. In most applications the stacks are already vertically aligned, however this is to allow air bubbles to freely flow out of the compartment [33].

Another interesting property of electroconvection is the (chaotic) mixing of the boundary layer. In microfluidic systems, like lab-on-a-chip applications [34], this phenomena can be used to make a tunable micromixers. On the micrometer scale mechanical fluid mixers are not practical or impossible and a turbulent flow is difficult to achieve due to the confinement. Alternatives have been proposed, such as the staggered herringbone structure [21]. A benefit of an electrical mixer is that it can be turned on and off $[35,36]$. With the increasing development in microfluidics the understanding of the interesting phenomenon of electroconvection is sure to find its place. But also in other systems with electric fields, charge selective interfaces and electrolytes the knowledge gained in this research might become useful, but surely first more research is needed. 


\section{Bibliography}

[1] S. Rubinstein, G. Manukyan, A. Staicu, I. Rubinstein, B. Zaltzman, R. Lammertink, F. Mugele, and M. Wessling, Direct Observation of a Nonequilibrium Electro-Osmotic Instability, Physical Review Letters 101, (2008). - p.140.

[2] I. Rubinstein and B. Zaltzman, Electro-osmotically induced convection at a permselective membrane, Physical Review E 62, 2238 (2000). — p.140.

[3] J. Balster, M. H. Yildirim, D. F. Stamatialis, R. Ibanez, R. G. H. Lammertink, V. Jordan, and M. Wessling, Morphology and microtopology of cation-exchange polymers and the origin of the overlimiting current., The journal of physical chemistry. B 111, 2152 (2007). — p.140, 141.

[4] S. M. Davidson, M. Wessling, and A. Mani, On the Dynamical Regimes of Pattern-Accelerated Electroconvection, Scientific Reports 6, 1 (2016). p.141, 142, 143, 144, 146.

[5] T. Femmer, A. J. C. Kuehne, J. Torres-Rendon, A. Walther, and M. Wessling, Print your membrane: Rapid prototyping of complex 3D-PDMS membranes via a sacrificial resist, Journal of Membrane Science 478, 12 (2015). — p.141.

[6] D. A. Vermaas, M. Saakes, and K. Nijmeijer, Power generation using profiled membranes in reverse electrodialysis, Journal of Membrane Science 385-386, 234 (2011). - p.141.

[7] M. Wessling, L. G. Morcillo, and S. Abdu, Nanometer-thick lateral polyelectrolyte micropatterns induce macrosopic electro-osmotic chaotic fluid instabilities., Scientific reports 4, 4294 (2014). — p.142, 146.

[8] S. Abdu, M.-C. Martí-Calatayud, J. E. Wong, M. García-Gabaldón, and M. Wessling, Layer-by-layer modification of cation exchange membranes controls ion selectivity and water splitting., ACS applied materials \& interfaces 6, 1843 (2014). - p.142.

[9] J. J. Krol, M. Wessling, and H. Strathmann, Concentration polarization with monopolar ion exchange membranes: currentvoltage curves and water dissociation, Journal of Membrane Science 162, 145 (1999). — p.142, 150.

[10] M. Taky, G. Pourcelly, and C. Gavach, Polarization phenomena at the interfaces between an electrolyte solution and an ion exchange membrane. Part II. 
Ion transfer with an anion exchange membrane, Journal of Electroanalytical Chemistry 336, 195 (1992). — p.142.

[11] D. E. Lide, CRC Handbook of Chemistry and Physics, 76th ed. (CRC Press, 1996), pp. 5-90. - p.142.

[12] V. S. Shelistov, E. A. Demekhin, and G. S. Ganchenko, Electrokinetic instability near charge-selective hydrophobic surfaces, Physical Review E - Statistical, Nonlinear, and Soft Matter Physics 90, 1 (2014). — p.142.

[13] C. L. Druzgalski, M. B. Andersen, and a. Mani, Direct numerical simulation of electroconvective instability and hydrodynamic chaos near an ion-selective surface, Physics of Fluids 25, 110804 (2013). — p.143, 145.

[14] E. a. Demekhin, N. V. Nikitin, and V. S. Shelistov, Direct numerical simulation of electrokinetic instability and transition to chaotic motion, Physics of Fluids 25, 122001 (2013). - p..

[15] V. S. Pham, Z. Li, K. M. Lim, J. K. White, and J. Han, Direct numerical simulation of electroconvective instability and hysteretic current-voltage response of a permselective membrane, Physical Review E 86, (2012). p.143.

[16] E. a. Demekhin, N. V. Nikitin, and V. S. Shelistov, Three-dimensional coherent structures of electrokinetic instability, Physical Review E 90, 013031 (2014). - p.143, 146.

[17] C. Druzgalski and A. Mani, Statistical analysis of electroconvection near an ion-selective membrane in the highly chaotic regime, Physical Review Fluids 1, 073601 (2016). - p.143, 146.

[18] R. Femmer, A. Mani, and M. Wessling, Ion transport through electrolyte/polyelectrolyte multi-layers., Scientific reports 5, 11583 (2015). p.144.

[19] E. Karatay, M. B. Andersen, M. Wessling, and A. Mani, Coupling between Buoyancy Forces and Electroconvective Instability near Ion-Selective Surfaces, Physical Review Letters 116, 194501 (2016). — p.145.

[20] R. Kwak, V. S. Pham, B. Kim, L. Chen, and J. Han, Enhanced Salt Removal by Unipolar Ion Conduction in Ion Concentration Polarization Desalination., Scientific reports 6, 25349 (2016). - p.145.

[21] A. D. Stroock, S. K. W. Dertinger, A. Ajdari, I. Mezic, H. A. Stone, and G. M. Whitesides, Chaotic mixer for microchannels, Science (New York, N.Y.) 295, 647 (2002). — p.146, 150.

[22] C. Cierpka and C. J. Kähler, Particle imaging techniques for volumetric three-component (3D3C) velocity measurements in microfluidics, Journal of Visualization 15, 1 (2012). - p.146.

[23] M. Raffel, C. Willert, and J. Kompenhans, Particle image velocimetry: a practical guide, 2nd ed. (Springer, 2007), p. 448. - p.146. 
[24] F. Scarano, Tomographic PIV: principles and practice, Measurement Science and Technology 24, 012001 (2012). - p.146.

[25] J. R. Lakowicz, Principles of fluorescence spectroscopy, 3rd ed. (Springer, 2013). - p.146.

[26] H. Kim, C. M. G. Piqué, J. S. Horwitz, H. Mattoussi, H. Murata, Z. H. Kafafi, D. B. Chrisey, H. Kim, and C. M. Gilmore, Electrical, optical, and structural properties of indiumtinoxide thin films for organic light emitting devices, Journal of Applied Physics 86, 6451 (1999). — p.146.

[27] V. V. Nikonenko, A. V. Kovalenko, M. K. Urtenov, N. D. Pismenskaya, J. Han, P. Sistat, and G. Pourcelly, Desalination at overlimiting currents: State-of-the-art and perspectives, Desalination 342, 85 (2014). — p.149.

[28] V. V. Nikonenko, N. D. Pismenskaya, E. I. Belova, P. Sistat, P. Huguet, G. Pourcelly, and C. Larchet, Intensive current transfer in membrane systems: Modelling, mechanisms and application in electrodialysis, Advances in Colloid and Interface Science 160, 101 (2010). — p.150.

[29] H. Strathmann, Electrodialysis, a mature technology with a multitude of new applications, Desalination 264, 268 (2010). — p.150.

[30] J. Stodollick, R. Femmer, M. Gloede, T. Melin, and M. Wessling, Electrodialysis of itaconic acid: A short-cut model quantifying the electrical resistance in the overlimiting current density region, Journal of Membrane Science 453, 275 (2014). - p.150.

[31] W. L. Lin, Y. T. Ker, and T. F. Lin, Experimental observation and conjugated heat transfer analysis of vortex flow development in mixed convection of air in a horizontal rectangular duct, International Journal of Heat and Mass Transfer 39, 3667 (1996). — p.150.

[32] E. Grandjean and P. a. Monkewitz, Experimental investigation into localized instabilities of mixed RayleighBénardPoiseuille convection, Journal of Fluid Mechanics 640, 401 (2009). — p.150.

[33] H. Strathmann, Membrane science and technology series, 1st editio ed. (Elsevier, 2004), No. 9. - p.150.

[34] T. M. Squires and S. R. Quake, Microfluidics: Fluid physics at the nanoliter scale, Reviews of Modern Physics 77, 977 (2005). — p.150.

[35] P.-H. Chiu, C.-C. Chang, and R.-J. Yang, Electrokinetic micromixing of charged and non-charged samples near nanomicrochannel junction, Microfluidics and Nanofluidics 14, 839 (2012). — p.150.

[36] M. Bazant and T. Squires, Induced-Charge Electrokinetic Phenomena: Theory and Microfluidic Applications, Physical Review Letters 92, 066101 (2004). - p.150. 



\section{Algemene Nederlandse samenvatting}

Het werk dat beschreven wordt in dit proefschrift gaat over het begrip en het gebruik van overlimiting current (OLC) in de nabijheid van ionenuitwisselingsmembranen. OLC is een grenslaag fenomeen waarin extra ionentransport naast ionen migratie en diffusie begint, waardoor de ionenstroom door het membraan verhoogt. Dit werk heeft zich gericht op advectief gedreven OLC bij kationenuitwisselingsmembranen, aangezien bij zo'n membraan water dissociatie tijdens OLC te verwaarlozen is. Advectie, de overdracht van materie of warmte door een verplaatsing van de vloeistof waar dit in aanwezig is, werd op twee manieren bij het membraan veroorzaakt. De eerste manier van advectie wordt veroorzaakt door de directe koppeling van het elektrische veld en de ionen in de geladen laag op het membraanoppervlakte. Aangezien er een netto lading op het oppervlakte aanwezig is, ontstaat er aan het grensvlak een netto stroom van ionen welke de vloeistof in beweging kan zetten, wanneer het elektrische veld groot genoeg is. Dit fenomeen heet elektroconvectie, waar de convectie verwijst naar de som van advectief en diffusief transport. De tweede manier van advectie wordt veroorzaakt door de concentratiegradiënt die ontstaat aan het ion selectieve membraan wanneer er een loodrecht elektrische veld op staat. De concentratiegradiënt resulteert in een dichtheidsgradiënt welke instabiel kan worden door opwaartse krachten (drijfkacht), waardoor het tweede type convectie begint, genaamd zwaartekracht convectie. Dit gebeurt alleen als de lichtere (ion verarmde) laag ligt onder de zwaardere (ion verrijkte) laag wat wordt aangeduid als de gravitationeel onstabiele oriëntatie.

Beide soorten convectie (elektro- en zwaartekracht) worden geïnduceerd in een transparante elektrochemische cel waarin de vloeistof initieel stil staat. De tijdafhankelijke elektrische weerstand over het membraan wordt gemeten, terwijl een constante elektrische stroom of een constant voltage verschil wordt opgelegd. Tegelijkertijd wordt de vloeistof stroming die ontstaat bekeken door video opnames te maken van de beweging van tracer deeltjes die in de vloeistof zijn opgelost. De beelden met de deeltjes worden geanalyseerd door de verplaatsing van individuele deeltjes te volgen en door gebruik te maken van particle image velocimetry (PIV) algoritmes welke de collectieve deeltjes verplaatsing bepalen. Deze methodes die deeltjes volgen, onthullen de start, groei en grootte van de advectie. Met een an- 
dere optische techniek genaamd fluorescence lifetime image microscopy (FLIM) kan het concentratie profiel worden bepaald. De combinatie van de hierboven beschreven technieken maakt het mogelijke om het ion transport via diffusie, migratie en advectie te bepalen en dit te vergelijken met theoretische en numerieke voorspellingen (Chapter 1 en 2).

De elektroconvectieve mengingslaag naast een vlak kationenuitwisselingsmembraan start nadat de grenslaag uitgeput is en een extra drempelvoltage wordt toegepast. De grenslaag wordt instabiel waardoor wervelstromingen ontstaan welke de grenslaag instabiliteit vergroten. Dit positieve terugkoppelingsmechanisme wordt de elektro-kinetische instabiliteit (EKI) genoemd. De PIV data laat een laag met lateraal bewegende wervels zien waarvan de hoogte en breedte ongeveer hetzelfde zijn. De hoogte van laag groeit terwijl het aantal individuele wervels vermindert. Alhoewel de mengingslaag met wervels groeit tot het vaste dikte blijven de individuele wervels chaotisch langs het membraan bewegen. Het instellen van een hogere elektrische stroomdichtheid resulteert in een dikkere mengingslaag. Het geleidingsvermogen van de mengingslaag is ongeveer constant in alle experimenten (Chapter 3). Dit heeft geleid tot de hypothese dat de mengingslaag een vaste gemiddelde concentratie heeft welke onafhankelijk is van de grootte, terwijl de stilstaande laag een concentratiegradiënt heeft. Deze hypothese is ondersteund door het meten van het concentratieprofiel in het system met FLIM (Chapter 4).

De ontwikkeling van het concentratie profiel in het systeem domein is ook voorspeld met een numeriek model gebaseerd op Ficks wetten van diffusie met constante flux randvoorwaardes in een stilstaande vloeistof. De modelvoorspellingen komen overeen met de gemeten concentratie profiel ontwikkeling en de voorspelde tijd waarop de grenslaag uitgeput raakt van ionen, op welk punt de elektroconvectie start. De theoretische depletietijd komt overeen met de elektrische transitietijd en het moment waarop elektroconvectie start zoals gezien in de gravitationeel stabiele oriëntatie waar geen zwaartekracht convectie kan ontstaan (Chapter 3 en 4). Deze oriëwordt de counter-oriëntatie genoemd, gezien het elektrische veld en het zwaartekracht veld in tegengesteld richting wijzen. In de co-oriëntatie wijzen beide velden naar de aarde en kan het model ook gebruikt worden om te voorspellen wanneer de concentratie gradiënt gravitationeel instabiel wordt en Rayleigh-Bénard (RB) convectie ontstaat. Om dit te testen wordt de cel in deze twee oriëntaties ten opzichte van het zwaartekracht veld doorgemeten wat laat zien dat de numerieke en experimentele transitie tijden in overeenstemming zijn. De metingen laten ook zien dat RB convectie ontstaat in de volledige cel wat de weerstand drastische vermindert. Bij hoge stroomdichtheden bestaan elektroconvectie via de elektro-kinetische instabiliteit (EKI) en RB convectie simultaan, gezien het additionele ion transport via RB niet genoeg is 
om te voorkomen dat de grenslaag volledig uitgeput raakt. De menging via RB vermindert de diffusie grenslaag waardoor het de EKI laag drastisch verkleint (Chapter 4). Dit is vergelijkbaar met de vermindering van de diffusie grenslaag door druk aangedreven vloeistofstroming.

De precieze koppeling tussen het elektrische veld en de EKI mengingslaag is een veel besproken vraag. Om de start van EKI te begrijpen zijn membranen met verschillende lijnstructuren vergeleken onder constante potentiaal condities. De aanwezigheid van de structuren reduceert het drempelvoltage dat nodig is om elektroconvectie te starten met $60 \%$ vergeleken met een vlak membraan (Chapter 5). De structuren werken als startpunten voor elektroconvectie wat resulteert in periodieke en stabiele wervels. Aan het vlakke membraan ontstaan de wervels op willekeurige plaatsen en bewegen lateraal langs het oppervlakte. Het gestructureerde membraanoppervlak heeft een deel dat parallel staat aan het externe elektrische veld waarlangs elektro-osmotische vloeistofstroom (wand EOV) ontstaat. Onze hypothesis is dat de wand EOV ontstaat zonder drempel en groeit in kracht terwijl de concentratie verder uit put en de geladen grenslaag uit zet. Dit leidt tot periodieke heterogeniteit in het concentratie profiel en het elektrische veld wat zorgt dat EKI kan starten bij een lagere drempelvoltage.

$\mathrm{Na}$ de start groeien de wervels tot een vaste grootte. Wanneer de membraanstructuren dezelfde dimensie hebben als de uiteindelijke dikte van de mengingslaag is de weerstand van de laag $50 \%$ lager vergeleken met een vlak membraan. De oppervlakte structuren fixeren de posities van de wervels, waardoor er stabiele instroomlocaties zijn met een hoge ionenconcentratie en uitstroomlocaties met een lage concentratie. De PIV en FLIM resultaten laten zien dat de menging bestaat uit rollen van wervels langs de lijnenstructuur (Chapter 5). Met dezelfde drijvende elektrische kracht kunnen de wervels groter groeien waardoor de mengingslaag verder van het membraan oppervlakte komt en vloeistof met een hogere ionenconcentratie naar het membraan brengt. Dit resulteert in een verhoogde gemiddelde concentratie in de mengingslaag en een lagere weerstand. Uit de weerstand, concentratie en vloeistofstroming data kan het ionentransport door middel van migratie, diffusie en advectie worden afgeschat. Deze berekeningen geven de goede orde van grootheid voor het transport van de ionen. Dit suggereert dat in de stabiele toestand geen andere grote transport processen significant zijn (e.g. het transport door andere ionen $\left(\mathrm{H}^{+}, \mathrm{OH}^{-}\right)$of het verlies van ionen door chemische reacties (copper oxide scaling)).

Het werk gepresenteerd in dit proefschrift beschrijf de observaties en bepalingen van ion concentration polarisation (ICP) door toepassing van technieken als fluorescence lifetime image microscopy (FLIM), particle tracking and particle image velocimetry (PIV). De laatste techniek is ook gebruikt om te bewijzen dat elektroconvectie een bron van overlimiting current (OLC) is bij een kationen- 
uitwisselingsmembraan. Een nieuw regime van chaotische vloeistofbeweging in de elektroconvectieve mengingslaag is gezien, in tegenstelling tot wat eerder beschreven is door andere onderzoekers. Een ander nieuw aspect van dit werk is de observatie en classificatie van de koppeling tussen electroconvectie en RayleighBénard convectie. Verder wordt in dit werk het gedrag van elektroconvectieve wervels bij geometrisch gestructureerde membranen gekwantificeerd. Wanneer de dikte van de mengingslaag en de periodiciteit van de structuren vergelijkbare grootte hebben stabiliseren de wervels, wat de weerstand van de laag vermindert. 


\section{Acknowledgements}

Like most things in life also writing a PhD thesis is not something you do alone. I am really grateful for the great scientific community that was around me and gave me access to lots of knowledge via literature, conferences and fellow scientists. Nice and smart people who helped me understand and reflect on my research topic. I am as well grateful for all the spontaneous non-scientific interaction. The moments where people made me laugh, relax, reflect, and consciously or unconsciously helped me grow as a person. Since everything is connected, I am grateful to everyone reading this, but there are some special people I would like to name.

First I want to thank my promoter and supervisor Rob for giving me the opportunity to work on this interesting project and for being always enthusiastic and relaxed. Never did I notice you letting your own stress negatively affect another person. This is a quality this world could use more. Also for the help of my other supervisors, I am grateful. Amy, for the patience and optimism and for teaching me to be a better researcher. Also thank you for the tea. Martijn, for the brainstorm sessions behind the whiteboard and the trips to the lab you always enjoyed. Jeff, for your knowledge, dedication, hospitality and for showing me best episodes of the Star Trek original series.

I would not have started this project without Sam telling me about a sustainable water technology research institute in Leeuwarden. The enthusiasm of Maarten made the application period go so fast and smooth and for this I am grateful. The wise words of the women on the train gave the final push to start this adventure I don't regret at all. She said: "ze bakken overal brood". So I moved to Leeuwarden and had a great start at the J. Semsstraat. Cycling, baking bread, watching Luffy, parties, rabbits and chickens. Thanks Sam!

When I arrived at Wetsus, I had the luck to be placed in the wonderful office HW 1.ooooooo6. Thank you David for your super dry humour (more quantity than quality), and your membrane and Matlab knowledge, and your time to answer questions while writing your thesis. Jordi, my fellow paranymph, and the master supplier of office humour via our app group. We started around the same time and I am glad you later joined me in the "depressing" room to close the cycle together. Pedro, senior and junior at the same time, the most funny 
office mate. The timing for your jokes is amazing and your mustache is also amazing. João, thank you for showing me the beautiful (weather of) Portugal and your hospitality. Monir, for being yourself and teaching me Iranian and naming our plant Moshkeli nist (meaning no problem) which just keeps growing. Elsemiek, short but unforgettable. And then Fabian came just in time to have one month of old building experience and know the noise cancellation device, the neighbour office poll, the small windows with curtain issues and the proper way to pronounce HW 1.06. We should watch a documentary together soon.

Regarding the science behind my project there are some people I want to thank. Slawek, that generously provided the potentiostat I used for all my experiments and taught me about resistances, how to build stuff in the workshop and to be a motivated scientist. Adam, for helping me work with optics and by knowing so much, it sometimes fried my brain. The days of physically building up the laserlab (twice) were awesome. All supporting people at Wetsus, but in particular Harm and Jan Jurjen who came up with better designs to my problems and taught me how to use the awesome machines in the werkplaats, and Rienk that helped me with the many IT issues. I am grateful for the help of Aziz and Morten who did great work as master students and gave my work the extra dimension of being a supervisor, which motivated and taught me a lot. Also the collaboration with scientists outside the Netherlands was inspiring and useful. Matthias, Robert, Saïd, Ali, Clara, Scott and Elif, thank you for your time and help!

I would also like to thank Johannes, Bert and Cees for the cooperative way in which they manage Wetsus and the jokes at the coffee machine. Henk, for organizing the team meetings and his interest in the project. And many thanks to the people from the UCF, now RUG/Campus Fryslân, for their effort to help their Frysian PhD's with courses and excursions.

The atmosphere at Wetsus is mostly a happy one and I want to thank all my colleagues for that. Some names in particular, Roel (BBQ host nr1), Taina (my life teacher), Charu (namaste), Jan G/P/T, Marianne/a, Janneke D/T, Philip $\mathrm{K} / \mathrm{W}$, Lina, Rik (effe knuffelen), Natascha (HW1.oo6 alumni without seeing that office), Terica (wah gwan - mandem mad), Marco, Aga (zet hem op), Ricardo (francesinha), Jaap (die bril staat je goed), Astrid (star trek dvd's), Dries (nice moustache), Jouke (iets grappigs), Pau (thanks for lending me books), Helena (recipes), Gerwin (veldhoven and the name of wind), Riet en Gerben (keukenhelden), Elmar (chifu), Hector (consistency), Michele, Paula (elfstedentocht). These people and more created awesome initiatives which gave me a lot of energy, for example the PV 2013, the MoWetsus tradition, the Salsa school, the cycling club, the pingpong committee, all $\mathrm{PhD}$ representatives, the laserlab gang, the climbing group.

Monthly I left on my journey to the University of Twente. I want to tank all 
my Twente colleagues for the interesting talks, jokes at the coffee table and at winterschool. I specially want to thank Damon (sansula), Sander, Yali, Anne (goede eerste indruk), Vic, Hanieh (nice couch), Khalid, Elif (first as mentor, later collaborator), Ingrid (your membranes made the project), Jan and Antoine (superman).

Buiten de wetenschappelijk wereld zijn er een aantal belangelijke mensen die mij hebben geholpen. Jan, Antsje, Doede, bedankt voor de hulp waardoor ik mezelf steeds weer beter leer kennen. De hele klimgroep en de 3J's, bedankt voor de carpoolritjes en de wekelijkse pret. Deze hobby neem ik mee, waar ik ook terecht kom. Ook mijn woongroep de Hoeksterpoort wil ik bedanken. Het mooiste aspect aan samen leven is dat iedereen zichzelf kan zijn en daarmee iets unieks brengt. Bedankt Andries, Jort, Jens, Renske, Agnes, Petra, Dirkje, Harry, Saskia, Keimpe Jan, Rene.

Ook alle mensen die niets met mijn Leeuwardentijd te maken hebben, wil ik bedanken voor alle gezelligheid en liefde. De straat, de groene hand, 3e kerstdag, 74/75/76, JUMP, konijn en natuurlijk mijn familie.

For my paranymfs, Jan Jurjen and Slawek, some last words: "Let's party!"

Finalment voldria agrair a la meva estimada Anna. Sense tu jo no podria haver fet això. El teu amor, compassió i determinació m’inspiren. És així! It is like that lieveling! 



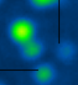

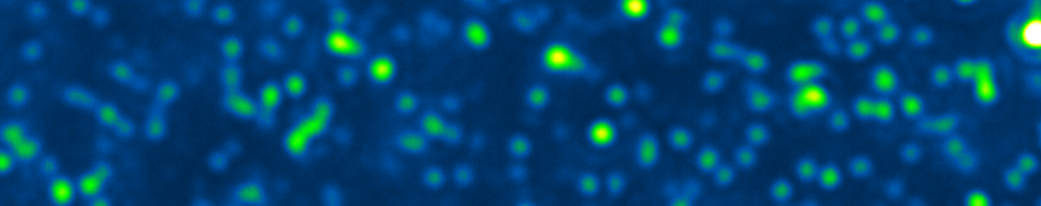

80

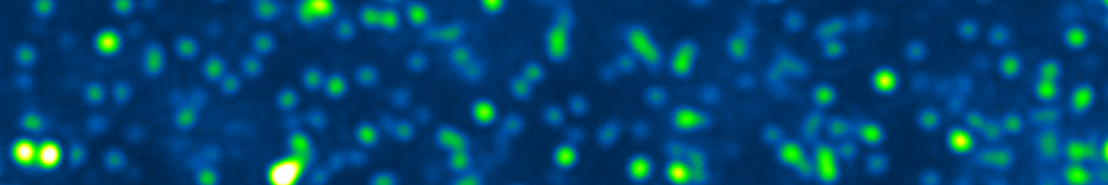

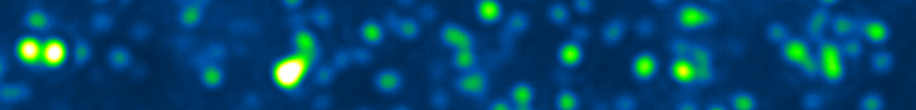

\section{6}

$c^{*}-4$

a

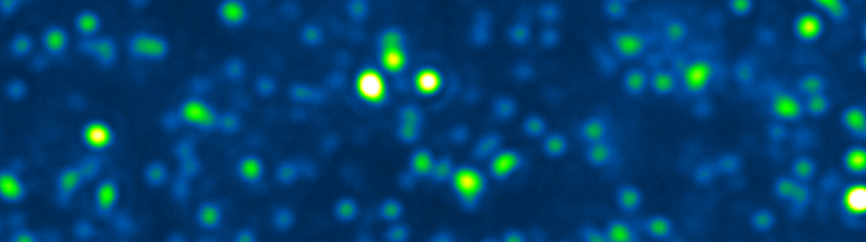

P.

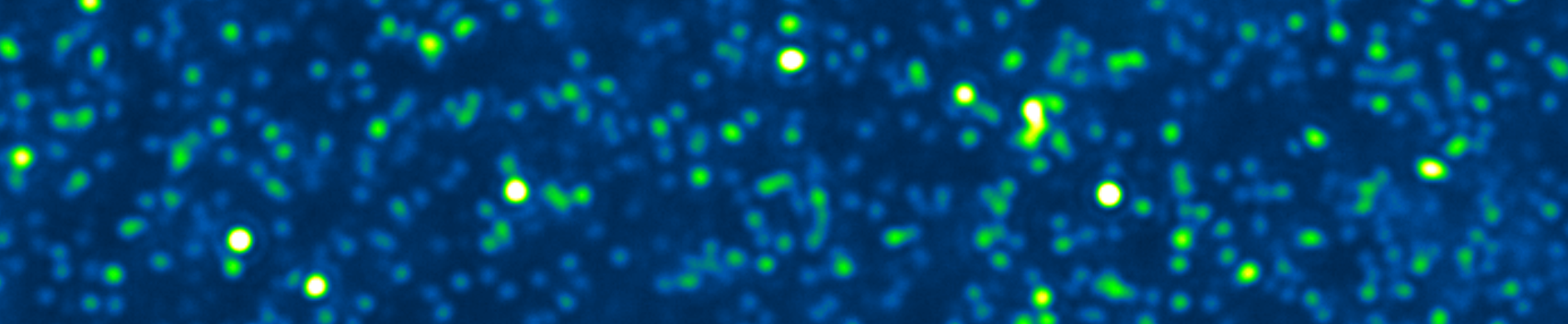

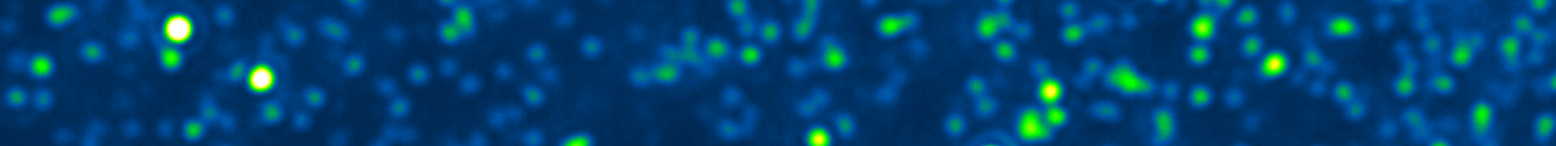
0
0

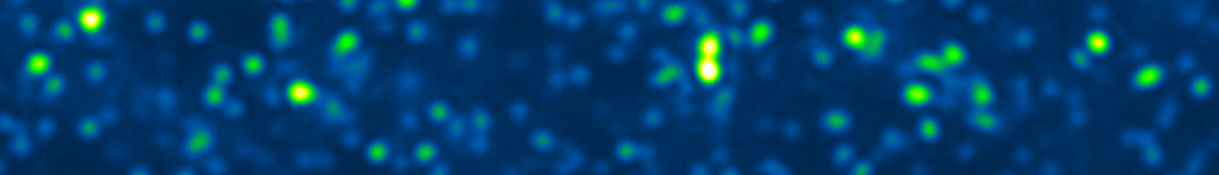

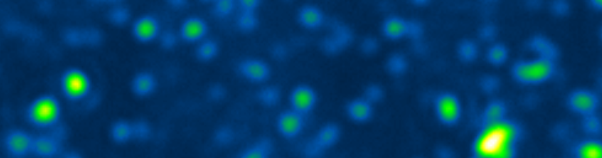

ISBN: 978-90-365-4314-9 